Supporting Information for the Paper entitled

\title{
Enantioselective Synthesis of Diversely Substituted Quaternary 1,4-Benzodiazepin-2-ones and 1,4-Benzodiazepin-2,5-diones
}

\author{
Paul R. Carlier*, Hongwu Zhao, Stephanie L. MacQuarrie-Hunter, \\ Joseph C. DeGuzman, Danny C. Hsu \\ Department of Chemistry \\ Virginia Tech \\ Blacksburg, VA 24061 \\ pcarlier@vt.edu
}

\section{Table of Contents:}

\begin{tabular}{|c|c|c|}
\hline Section & Description & Page \\
\hline A. & Full citations for references 1 and 21 in the paper & $\mathrm{S} 1$ \\
\hline B. & General Experimental & $\mathrm{S} 2$ \\
\hline C. & Preparation of $N$-DAM 1,4-benzodiazepin-2-ones & $\mathrm{S} 2$ \\
\hline D. & $\begin{array}{l}\text { Enantioselective deprotonation/alkylation of } N \text {-DAM 1,4- } \\
\text { benzodiazepin-2-ones }\end{array}$ & S5 \\
\hline E. & $\begin{array}{l}\text { Removal of DAM group from quaternary } N \text {-DAM 1,4-benzodiazepin-2- } \\
\text { ones }\end{array}$ & $\mathrm{S} 18$ \\
\hline F. & $\begin{array}{l}\text { Hydrolysis of quaternary } N \text {-H 1,4-benzodiazepin-2-ones to the } \\
\text { corresponding quaternary amino acids }\end{array}$ & $\mathrm{S} 21$ \\
\hline G. & $\begin{array}{l}\text { Synthesis of } N \text {-DAM 1,4-benzodiazepin-2,5-diones derived from } \\
\text { proline, thioproline, and 4-hydroxyproline }\end{array}$ & $\mathrm{S} 22$ \\
\hline H. & $\begin{array}{l}\text { Enantioselective deprotonation/alkylation of } N \text {-DAM 1,4- } \\
\text { benzodiazepin-2,5-diones derived from proline, thioproline, and 4- } \\
\text { hydroxyproline }\end{array}$ & $\mathrm{S} 24$ \\
\hline I. & $\begin{array}{l}\text { Removal of DAM group from quaternary } N \text {-DAM 1,4-benzodiazepin- } \\
\text { 2,5-diones }\end{array}$ & $\mathrm{S} 28$ \\
\hline $\mathrm{J}$. & Table of HPLC retention times & S29 \\
\hline K. & References & $\mathrm{S} 32$ \\
\hline L. & $\begin{array}{l}{ }^{1} \mathrm{H} \text { NMR spectra for new compounds that lack HPLC analysis: } 21 \mathrm{c}, \mathbf{3 4 c} \text {, } \\
\text { 33d, 34d, 35c, 37c, 38c }\end{array}$ & $\mathrm{S} 33$ \\
\hline
\end{tabular}

\section{A. Full citations for references 1 and 21 of paper}

Reference 1: Evans, B. E.; Rittle, K. E.; Bock, M. G.; DiPardo, R. M.; Freidinger, R. M.; Whitter, W. L.; Lundell, G. F.; Veber, D. F.; Anderson, P. S.; Chang, R. S. L.; Lotti, V. J.; Cerino, D. J.; Chen, T. B.; Kling, P. J.; Kunkel, K. A.; Springer, J. P.; Hirschfield, J. J. Med. Chem. 1988, 31, 2235-2246.

Reference 21: Selnick, H. G.; Liverton, N. J.; Baldwin, J. J.; Butcher, J. W.; Claremon, D. A.; Elliott, J. M.; Freidinger, R. M.; King, S. A.; Libby, B. E.; McIntyre, C. J.; Pribush, D. A.; Remy, D. C.; Smith, G. R.; Tebben, A. J.; Juriewicz, N. K.; Lynch, J. J.; Salata, J. J.; Sanguinetti, M. C.; Siegl, P. K. S.; Slaughter, D. E.; Vyas, K. J. Med. Chem. 1997, 40, 3865-3868. 


\section{B. General Experimental Section}

${ }^{1} \mathrm{H}$ NMR Spectra were recorded at 500 and $400 \mathrm{MHz}$; the corresponding ${ }^{13} \mathrm{C}$ NMR resonant frequencies were 125 and $100 \mathrm{MHz}$ respectively. High resolution mass spectra were recorded under FAB conditions (NBA. PEG); in each case the expected molecular formula $\left(\mathrm{M}+1,{ }^{35} \mathrm{Cl}\right)$ gave the closest match among all possible formulas. In conjunction with the ${ }^{1} \mathrm{H}$ and ${ }^{13} \mathrm{C}$ NMR spectra the HRMS data confirm the proposed identity of each compound. Chiral stationary phase HPLC analysis was performed on the great majority of new compounds and indicated $>99$ area\% chemical purity for these compounds. For the few compounds lacking HPLC analysis, ${ }^{1} \mathrm{H}$ NMR spectra are attached as evidence of purity. THF and DME were distilled from $\mathrm{Na} /$ benzophenone immediately before use. Enantiomeric excess of all compounds were assessed by HPLC (Chiralcel AD and OD). (S)-Boc-amino acids were purchased from Advanced ChemTech; cis-4-hydroxy-D-proline and trans-4-hydroxy-L-proline were purchased from Aldrich. Known compounds $(S)-1 \mathbf{c}^{1,2}$ and $(S)-5 \mathbf{c},{ }^{3}$ were prepared in $91 \%$ and $64 \%$ yield from (S)-Boc-Ala-OH and 2-amino-chlorobenzophenone or 2-aminobenzophenone respectively, using a slight modification of Shea's protocol. ${ }^{2}$ This modification consisted of a use of a buffered $\mathrm{pH} 7$ solution to effect the cyclization step; in our hands this gave faster reaction and less racemization relative to the $\mathrm{pH} 8.5$ conditions recommended by Shea. Known compounds $(S)-6 \mathbf{c}^{1,2}(73 \%),(S)$ $7 \mathbf{c}^{4}(69 \%),(S)-8 \mathbf{c}^{4}(61 \%)$ were similarly prepared from 2-amino-5-chlorobenzophenone and $(S)$ Boc-Phe-OH, $(S)$-Boc-Abu-OH, and $(S)$-Boc-Met-OH, respectively. Known 1,4-benzodiazepin2,5 -diones $(S)-\mathbf{3 c}^{5}(75 \%),(S)-3 \mathbf{3 0} \mathbf{c}^{6}(87 \%),(2 R, 11 \mathrm{a} R)-31 \mathbf{c}^{7}(65 \%)$, and $(2 R, 11 \mathrm{a} S)-32 \mathbf{c}^{8}(77 \%)$ were prepared by heating the corresponding proline derivative with isatoic anhydride in DMSO at $120-140{ }^{\circ} \mathrm{C}$ for $2-5$ hours. HPLC analysis indicated $>98 \%$ ee for each of the $N-\mathrm{H} 1,4-$ benzodiazepin-2-ones and $\mathrm{N}$-H-1,4-benzodiazepin-2,5-diones.

\section{Preparation of $N$-DAM 1,4-benzodiazepin-2-ones}

\section{Di(p-anisyl)methyl bromide (DAM-Br)}

At room temperature, to a stirred solution of 4,4'-Dimethoxybenzhydrol $(4.8 \mathrm{~g}, 19.8 \mathrm{mmol})$ in anhydrous benzene $(45 \mathrm{~mL})$ was added acetyl bromide $(6.6 \mathrm{~mL}, 87.0 \mathrm{mmol})$ dropwise by syringe. After addition, the resulting solution was stirred for another $1.0 \mathrm{~h}$. The benzene was removed in vacuo below $40^{\circ} \mathrm{C}$, and the resulting residue was dried under high vacuum for $1 \mathrm{~h}$, with cooling of the sample with liquid $\mathrm{N}_{2}$. The resulting pale solid was crystallized from dried $\mathrm{n}$ hexane to afford $5.8 \mathrm{~g}(95 \%)$ of DAM-Br as a white solid.

${ }^{1} \mathrm{H} \mathrm{NMR}\left(\mathrm{CDCl}_{3}\right) \delta$ 7.41-7.35 (m, 4H), 6.90-6.82 (m, 4H), 6.30 (s, 1H), $3.80(\mathrm{~s}, 6 \mathrm{H})$.

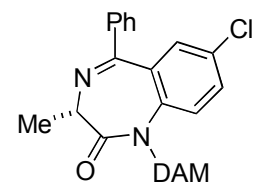

(3S)-(+)-1-di(p-anisyl)methyl-7-chloro-1,3-dihydro-3-methyl-5-phenyl-2H-1,4benzodiazepin-2-one $[(S)-(+)-1 \mathrm{~d}]$

At $0{ }^{\circ} \mathrm{C}$ to a stirred mixture of compound $(S)-1 \mathbf{c}(2.8 \mathrm{~g}, 9.98 \mathrm{mmol}, 1.0$ equiv.) and HMPA (12.9 $\mathrm{mL}, 59.3 \mathrm{mmol}, 6.0$ equiv) in anhydrous THF $(100 \mathrm{~mL})$ was added $\mathrm{NaH}(593.3 \mathrm{mg}, 14.8 \mathrm{mmol}$, 1.5 equiv., $60 \%$ suspension in mineral oil) in one portion. The resulting solution was stirred at 0 ${ }^{\circ} \mathrm{C}$ for 30 min before a solution of DAM-Br $(6.1 \mathrm{~g}, 9.8 \mathrm{mmol}, 2.0$ equiv.) in anhydrous THF (20 
$\mathrm{ml}$ ) was added dropwise via a syringe. The reaction mixture was stirred for a further $2.0 \mathrm{~h}$ at $0^{\circ} \mathrm{C}$, at which point TLC (1:3 EtOAc / hexanes) indicated the reaction was complete. The reaction was quenched with $40 \mathrm{~mL}$ of sat. $\mathrm{NH}_{4} \mathrm{Cl}(\mathrm{aq})$, and extracted with $\mathrm{CH}_{2} \mathrm{Cl}_{2}(3 \times 100 \mathrm{~mL})$. The combined organic extracts were dried over anhydrous $\mathrm{Na}_{2} \mathrm{SO}_{4}$, filtered, and concentrated under reduced pressure. The crude product was purified with flash column chromatography on silica gel (eluent: ethyl acetate/n-Hexane $=1: 4)$ to afford $(S)-(+)-\mathbf{1 d}$ as white solid. Yield: 75\%, m.p. 66.5-66.9 ${ }^{\circ} \mathrm{C},[\alpha]_{\mathrm{D}}{ }^{23}=+92.8\left(\mathrm{c}=1.3, \mathrm{CHCl}_{3}\right) .{ }^{1} \mathrm{H}$ NMR $\left(\mathrm{CDCl}_{3}\right): \delta$ 7.49-7.40 (m, $\left.1 \mathrm{H}\right), 7.39-7.30$ (m, 2H), 7.29-7.22 (m, 5H), 7.18-7.10 (m, 1H), 7.09-7.00 (m, 2H), 6.97-6.87 (m, 4H), $6.63(\mathrm{~d}, J$ $=8.5 \mathrm{~Hz}, 2 \mathrm{H}), 3.92(\mathrm{q}, J=6.4 \mathrm{~Hz}, 1 \mathrm{H}), 3.83(\mathrm{~s}, 3 \mathrm{H}), 3.75(\mathrm{~s}, 3 \mathrm{H}), 1.77(\mathrm{~d}, J=6.4 \mathrm{~Hz}, 3 \mathrm{H}) .{ }^{13} \mathrm{C}$ $\mathrm{NMR}\left(\mathrm{CDCl}_{3}\right): \delta 170.8,167.0,159.0,158.8,139.7,137.9,133.3,131.4,130.5,130.4,130.2$, 130.0, 129.5, 129.0, 128.9, 128.3, 127.8, 126.1, 114.0, 113.9, 113.7, 64.0, 59.4, 55.4, 55.3, 17.5. HRMS calcd. for $\mathrm{C}_{31} \mathrm{H}_{28} \mathrm{ClN}_{2} \mathrm{O}_{3}(\mathrm{M}+1) 511.1788$, found 511.1772 (-3.2 ppm, $\left.-1.7 \mathrm{mmu}\right)$. HPLC $\mathrm{t}_{\mathrm{r}} 10.6 \mathrm{~min}(R) ; \mathrm{t}_{\mathrm{r}} 16.4 \mathrm{~min}(S)$ [Chiralpak $\mathrm{AD}(0.46 \mathrm{~cm} \times 25 \mathrm{~cm})$ (from Daicel Chemical Ind., Ltd.)] Hexane/i-PrOH, 90/10, $1.0 \mathrm{~mL} / \mathrm{min}, 100 \%$ ee.

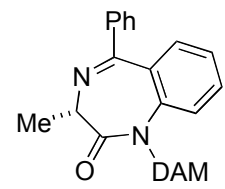

(3S)-(+)-1-di( $p$-anisyl)methyl-1,3-dihydro-3-methyl-5-phenyl-2H-1,4-benzodiazepin-2-one $[(S)-(+)-5 d]$

At $0^{\circ} \mathrm{C}$ to a stirred mixture of compound $(S)-5 \mathbf{c}(0.500 \mathrm{~g}, 2.0 \mathrm{mmol}, 1.0$ equiv. $)$ in anhydrous THF (12 mL) was added $\mathrm{NaH}$ (160 mg, $4.0 \mathrm{mmol}, 2.0$ equiv., 60\% suspension in mineral oil) in one portion. The resulting solution was stirred for $30 \mathrm{~min}$. DAM-Br (1.23 g, $4.0 \mathrm{mmol}, 2.0$ equiv.) was added to the solution. The reaction mixture was stirred for a further $4 \mathrm{~h}$ at $0{ }^{\circ} \mathrm{C}$, at which point TLC (3:7 EtOAc / hexanes) indicated the reaction was complete. The reaction was quenched with $10 \mathrm{~mL} \mathrm{H} \mathrm{H}_{2} \mathrm{O}$ and extracted with $\mathrm{CH}_{2} \mathrm{Cl}_{2}(3 \times 10 \mathrm{~mL})$. The combined organic extracts were dried over $\mathrm{Na}_{2} \mathrm{SO}_{4}$, filtered, and concentrated under reduced pressure. The crude product was purified with flash column chromatography on silica gel $\left(1^{\text {st }}\right.$ eluent: ethyl acetate $/ n$ hexane $=35: 65,2^{\text {nd }}$ eluent: $\left.\mathrm{Et}_{2} \mathrm{O} / \mathrm{DCM}=1: 15\right)$ to afford $(S)-(+)-5 d$ as white foam. $0.6717 \mathrm{~g}$, yield $71 \%$. When 1.2 equiv of $\mathrm{NaH}$ was used in the reaction, the yield was slightly lower $(66 \%)$. $[\alpha]_{\mathrm{D}}{ }^{25}=+182\left(c=0.48, \mathrm{CHCl}_{3}\right) .{ }^{1} \mathrm{H} \mathrm{NMR}\left(\mathrm{CDCl}_{3}\right) \delta 1.78\left(\mathrm{~d},{ }^{3} J_{\mathrm{HH}}=6.5 \mathrm{~Hz}, 3 \mathrm{H}\right), 3.74(\mathrm{~s}, 3 \mathrm{H})$, $3.84(\mathrm{~s}, 3 \mathrm{H}), 3.94\left(\mathrm{q},{ }^{3} J_{\mathrm{HH}}=6.5 \mathrm{~Hz}, 1 \mathrm{H}\right), 6.62\left(\mathrm{~d},{ }^{3} J_{\mathrm{HH}}=8.5 \mathrm{~Hz}, 2 \mathrm{H}\right), 6.91-6.95(\mathrm{~m}, 4 \mathrm{H}), 7.02-$ $7.10(\mathrm{~m}, 3 \mathrm{H}), 7.20\left(\mathrm{t},{ }^{3} J_{\mathrm{HH}}=8.5 \mathrm{~Hz}, 1 \mathrm{H}\right), 7.26-7.34(\mathrm{~m}, 7 \mathrm{H}), 7.42\left(\mathrm{t},{ }^{3} J_{\mathrm{HH}}=7.0 \mathrm{~Hz}, 1 \mathrm{H}\right) ;{ }^{13} \mathrm{C}$ $\operatorname{NMR}\left(\mathrm{CDCl}_{3}\right)$ (25 resonances found for a possible 25 unique carbons): $\delta 17.57,55.28,55.42$, 59.27, 64.14, 113.49, 113.83, 124.67, 124.76, 128.09, 129.04, 129.55, 129.62, 129.94, 130.25, 130.73, 131.00, 131.44, 131.97, 138.60, 141.17, 158.67, 158.89, 168.33, 171.19. HRMS (FAB) calcd for $\mathrm{C}_{31} \mathrm{H}_{29} \mathrm{~N}_{2} \mathrm{O}_{3}[\mathrm{M}+\mathrm{H}]^{+}$: 477.2178. Found: $477.2161(-3.6 \mathrm{ppm},-1.7 \mathrm{mmu})$. HPLC $\mathrm{t}_{\mathrm{r}} 16.3$ $\min (R) ; \mathrm{t}_{\mathrm{r}} 19.7 \mathrm{~min}(S)$ [Chiralcel OD $(0.46 \mathrm{~cm} \times 25 \mathrm{~cm})$ (from Daicel Chemical Ind., Ltd.) Hexane $/ i-\mathrm{PrOH}, 3 / 97 \mathrm{~mL} / \mathrm{min},>99.5 \%$ ee.<smiles>CON1C(=O)[C@H](Cc2ccccc2)N=C(c2ccccc2)c2cc(Cl)ccc21</smiles>

(3S)-(+)-1-di(p-anisyl)methyl-3-benzyl-7-chloro-1,3-dihydro-5-phenyl-2H-1,4benzodiazepin-2-one $[(S)-(+)-6 \mathrm{~d}]$ 
At $0{ }^{\circ} \mathrm{C}$ to a stirred mixture of compound $(S)-6 \mathrm{c}(0.1000 \mathrm{~g}, 0.277 \mathrm{mmol}, 1.0$ equiv. $)$ in anhydrous THF ( $2 \mathrm{~mL})$ was added $\mathrm{NaH}(21.8 \mathrm{mg}, 0.554 \mathrm{mmol}, 2.0$ equiv., $60 \%$ suspension in mineral oil) in one portion. The resulting solution was stirred for $30 \mathrm{~min}$. DAM-Br $(0.1702 \mathrm{~g}, 0.554 \mathrm{mmol}$, 2.0 equiv.) was added to the solution. The reaction mixture was stirred for a further $4 \mathrm{~h}$ at $0^{\circ} \mathrm{C}$, at which point TLC (3:7 EtOAc / hexanes) indicated the reaction was complete. The reaction was quenched with $5 \mathrm{~mL} \mathrm{H} \mathrm{H}_{2} \mathrm{O}$ and extracted with $\mathrm{CH}_{2} \mathrm{Cl}_{2}(3 \times 5 \mathrm{~mL})$. The combined organic extracts were dried over $\mathrm{Na}_{2} \mathrm{SO}_{4}$, filtered, and concentrated under reduced pressure. The crude product was purified with flash column chromatography on silica gel $\left(1^{\text {st }}\right.$ eluent: $\mathrm{Et}_{2} \mathrm{O} / \mathrm{DCM}=1: 20,2^{\text {nd }}$ eluent: ethyl acetate $/ n$-hexane=1:4) to afford $(S)-(+)-6 \mathbf{d}$ as colorless oil. $0.1314 \mathrm{~g}$, yield $83 \%$. When 1.2 equiv of $\mathrm{NaH}$ was used in the reaction, the yield was slightly lower $(75 \%) .[\alpha]_{\mathrm{D}}{ }^{25}=$ $+101\left(c=1.25, \mathrm{CHCl}_{3}\right) .{ }^{1} \mathrm{H}$ NMR $\left(\mathrm{CDCl}_{3}\right) \delta 3.65\left(\mathrm{dd},{ }^{2} J_{\mathrm{HH}}=46.0 \mathrm{~Hz},{ }^{3} J_{\mathrm{HH}}=17.8 \mathrm{~Hz}, 2 \mathrm{H}\right), 3.75$ $(\mathrm{s}, 3 \mathrm{H}), 3.84(\mathrm{~s}, 3 \mathrm{H}), 3.95\left(\mathrm{q},{ }^{3} J_{\mathrm{HH}}=5.5 \mathrm{~Hz}, 1 \mathrm{H}\right), 6.64\left(\mathrm{~d},{ }^{3} J_{\mathrm{HH}}=9.0 \mathrm{~Hz}, 3 \mathrm{H}\right), 6.90-6.93(\mathrm{~m}, 5 \mathrm{H})$, $6.99\left(\mathrm{~d},{ }^{4} J_{\mathrm{HH}}=2.5 \mathrm{~Hz}, 1 \mathrm{H}\right), 7.06(\mathrm{~s}, 1 \mathrm{H}), 7.10-7.17(\mathrm{~m}, 2 \mathrm{H}), 7.21-7.38(\mathrm{~m}, 9 \mathrm{H}), 7.44\left(\mathrm{t},{ }^{3} J_{\mathrm{HH}}=\right.$ $7.5 \mathrm{~Hz}, 1 \mathrm{H}) ;{ }^{13} \mathrm{C} \mathrm{NMR}\left(\mathrm{CDCl}_{3}\right)$ (29 resonances found for a possible 29 unique carbons): $\delta$ $38.14,55.45,55.59,64.14,65.99,113.84,113.93,114.11,126.18,126.43,128.46,128.49$, $129.09,129.34,129.72,130.09,130.23,130.39,130.47,130.82,131.51,133.13,138.10,139.58$, 139.68, 158.98, 159.21, 167.47, 169.92. HRMS (FAB) calcd for $\mathrm{C}_{37} \mathrm{H}_{32} \mathrm{~N}_{2} \mathrm{O}_{3} \mathrm{Cl}[\mathrm{M}+\mathrm{H}]^{+}$: 587.2101. Found: $587.2120(+3.1 \mathrm{ppm},+1.8 \mathrm{mmu})$. HPLC $\mathrm{t}_{\mathrm{r}} 40.2 \mathrm{~min}(S) ; \mathrm{t}_{\mathrm{r}} 46.7 \mathrm{~min}(R)$ [Chiralcel OD $(0.46 \mathrm{~cm} \times 25 \mathrm{~cm})$ (from Daicel Chemical Ind., Ltd.) Hexane $i-\mathrm{PrOH}, 99 / 1,1.0$ $\mathrm{mL} / \mathrm{min},>99.5 \%$ ee.

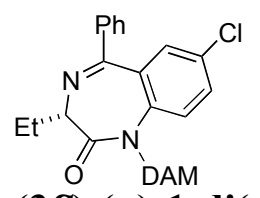

(3S)-(+)-1-di(p-anisyl)methyl-7-chloro-3-ethyl-1,3-dihydro-5-phenyl-2H-1,4-benzodiazepin2-one $[(S)-(+)-7 d]$

At $0{ }^{\circ} \mathrm{C}$ to a stirred mixture of compound $(S)-7 \mathrm{c}(0.100 \mathrm{~g}, 0.335 \mathrm{mmol}, 1.0$ equiv. $)$ in anhydrous THF (2 mL) was added $\mathrm{NaH}(27 \mathrm{mg}, 0.670 \mathrm{mmol}, 2.0$ equiv., $60 \%$ suspension in mineral oil $)$ in one portion. The resulting solution was stirred for $30 \mathrm{~min}$. DAM-Br $(0.206 \mathrm{~g}, 0.670 \mathrm{mmol}, 2.0$ equiv.) was added to the solution. The reaction mixture was stirred for a further $3 \mathrm{~h}$ at $0^{\circ} \mathrm{C}$, at which point TLC (3:7 EtOAc / hexanes) indicated the reaction was complete. The reaction was quenched with $5 \mathrm{~mL} \mathrm{H}_{2} \mathrm{O}$ and extracted with $\mathrm{CH}_{2} \mathrm{Cl}_{2}(3 \times 5 \mathrm{~mL})$. The combined organic extracts were dried over $\mathrm{Na}_{2} \mathrm{SO}_{4}$, filtered, and concentrated under reduced pressure. The crude product was purified with flash column chromatography on silica gel $\left(1^{\text {st }}\right.$ eluent: $\mathrm{Et}_{2} \mathrm{O} / \mathrm{DCM}=1: 20,2^{\text {nd }}$ eluent: ethyl acetate $/ n$-hexane $=1: 4)$ to afford $(S)-(+)-7 \mathbf{d}$ as white foam. $0.1687 \mathrm{~g}$, yield $96 \%$. When 1.2 equiv of $\mathrm{NaH}$ was used in the reaction, the yield was slightly lower $(81 \%)$. $[\alpha]_{\mathrm{D}}{ }^{25}=$ $+186\left(c=1.46, \mathrm{CHCl}_{3}\right) .{ }^{1} \mathrm{H} \mathrm{NMR}\left(\mathrm{CDCl}_{3}\right) \delta 1.07\left(\mathrm{t},{ }^{3} J_{\mathrm{HH}}=7.5 \mathrm{~Hz}, 3 \mathrm{H}\right), 2.26-2.36(\mathrm{~m}, 2 \mathrm{H}), 3.61$ $\left(\mathrm{t},{ }^{3} J_{\mathrm{HH}}=7.0 \mathrm{~Hz}, 1 \mathrm{H}\right), 3.76(\mathrm{~s}, 3 \mathrm{H}), 3.84(\mathrm{~s}, 3 \mathrm{H}), 6.64\left(\mathrm{~d},{ }^{3} J_{\mathrm{HH}}=8.5 \mathrm{~Hz}, 2 \mathrm{H}\right), 6.91-6.93(\mathrm{~m}, 4 \mathrm{H})$, $7.05(\mathrm{~s}, 1 \mathrm{H}), 7.07\left(\mathrm{~d},{ }^{4} J_{\mathrm{HH}}=2.5 \mathrm{~Hz}, 1 \mathrm{H}\right), 7.13-7.21(\mathrm{~m}, 2 \mathrm{H}), 7.24-7.29(\mathrm{~m}, 4 \mathrm{H}), 7.35\left(\mathrm{t},{ }^{3} J_{\mathrm{HH}}=\right.$ $7.5 \mathrm{~Hz}, 2 \mathrm{H}), 7.44\left(\mathrm{t},{ }^{3} \mathrm{~J}_{\mathrm{HH}}=7.5 \mathrm{~Hz}, 1 \mathrm{H}\right) ;{ }^{13} \mathrm{C} \mathrm{NMR}\left(\mathrm{CDCl}_{3}\right)$ (26 resonances found for a possible 26 unique carbons): $\delta 10.98,24.95,55.44,55.58,63.92,65.87,113.81,114.11,126.19,128.44$, 129.08, 129.21, 129.67, 130.11, 130.27, 130.53, 130.66, 130.72, 131.52, 133.34, 138.20, 139.87, 158.94, 159.17, 167.44, 170.23. HRMS (FAB) calcd for $\mathrm{C}_{32} \mathrm{H}_{30} \mathrm{~N}_{2} \mathrm{O}_{3} \mathrm{Cl}[\mathrm{M}+\mathrm{H}]^{+}: 525.1945$. Found: $525.1938(-1.4 \mathrm{ppm},-0.7 \mathrm{mmu})$. HPLC $\mathrm{t}_{\mathrm{r}} 25.1 \mathrm{~min}(R) ; \mathrm{t}_{\mathrm{r}} 28.9 \mathrm{~min}(S)$ [Chiralcel OD 
$(0.46 \mathrm{~cm} \times 25 \mathrm{~cm})($ from Daicel Chemical Ind., Ltd.) Hexane $/ i-\mathrm{PrOH}, 99 / 1,1.0 \mathrm{~mL} / \mathrm{min},>94 \%$ ee.<smiles>COCCC1N=C(c2ccccc2)c2cc(Cl)ccc2N1OC</smiles>

(3S)-(-)-1-di(p-anisyl)methyl-7-chloro-1,3-dihydro-5-phenyl-3-(2-thiomethyl)ethyl-2H-1,4benzodiazepin-2-one $[(S)-(-)-8 d]$

At $0{ }^{\circ} \mathrm{C}$ to a stirred mixture of compound $(S)-8 \mathrm{c}(0.100 \mathrm{~g}, 0.290 \mathrm{mmol}, 1.0$ equiv) in anhydrous THF ( $2 \mathrm{~mL}$ ) was added $\mathrm{NaH}$ (23 $\mathrm{mg}, 0.580 \mathrm{mmol}, 2.0$ equiv, 60\% suspension in mineral oil) in one portion. The resulting solution was stirred for $30 \mathrm{~min}$. DAM-Br $(0.1782 \mathrm{~g}, 0.580 \mathrm{mmol}, 2.0$ equiv) was added to the solution. The reaction mixture was stirred for a further $4 \mathrm{~h}$ at $0^{\circ} \mathrm{C}$, at which point TLC (3:7 EtOAc / hexanes) indicated the reaction was complete. The reaction was quenched with $5 \mathrm{~mL} \mathrm{H} \mathrm{H}_{2} \mathrm{O}$ and extracted with $\mathrm{CH}_{2} \mathrm{Cl}_{2}(3 \times 5 \mathrm{~mL})$. The combined organic extracts were dried over $\mathrm{Na}_{2} \mathrm{SO}_{4}$, filtered, and concentrated under reduced pressure. The crude product was purified with flash column chromatography on silica gel (eluent: $\mathrm{Et}_{2} \mathrm{O} / \mathrm{DCM}=1: 20$, three times) to afford $(S)-(-)-8 d$ as white foam. $0.0931 \mathrm{~g}$, yield $56 \%,[\alpha]_{\mathrm{D}}{ }^{25}=-127\left(c=0.86, \mathrm{CHCl}_{3}\right)$. ${ }^{1} \mathrm{H}$ NMR $\left(\mathrm{CDCl}_{3}\right) \delta 2.12(\mathrm{~s}, 3 \mathrm{H}), 2.55-2.64(\mathrm{~m}, 2 \mathrm{H}), 2.72-2.84(\mathrm{~m}, 2 \mathrm{H}), 3.75(\mathrm{~s}, 3 \mathrm{H}), 3.84(\mathrm{~s}$, $3 \mathrm{H}), 3.95\left(\mathrm{t},{ }^{3} J_{\mathrm{HH}}=8.3 \mathrm{~Hz}, 1 \mathrm{H}\right), 6.64\left(\mathrm{~d},{ }^{3} J_{\mathrm{HH}}=11.0 \mathrm{~Hz}, 2 \mathrm{H}\right), 6.90-6.95(\mathrm{~m}, 4 \mathrm{H}), 7.03(\mathrm{~s}, 1 \mathrm{H})$, $7.07\left(\mathrm{~d},{ }^{4} J_{\mathrm{HH}}=3.5 \mathrm{~Hz}, 1 \mathrm{H}\right), 7.14-7.23(\mathrm{~m}, 2 \mathrm{H}), 7.25-7.29(\mathrm{~m}, 4 \mathrm{H}), 7.36\left(\mathrm{t},{ }^{3} J_{\mathrm{HH}}=9.5 \mathrm{~Hz}, 2 \mathrm{H}\right)$, $7.45\left(\mathrm{t},{ }^{3} \mathrm{~J}_{\mathrm{HH}}=9.5 \mathrm{~Hz}, 1 \mathrm{H}\right) ;{ }^{13} \mathrm{C} \mathrm{NMR}\left(\mathrm{CDCl}_{3}\right)$ (26 resonances found for a possible 27 unique carbons): $\delta 15.78,31.01,31.03,55.44,55.56,62.70,64.10,113.83,114.12,126.28,128.48$, $129.07,129.25,129.67,130.29$, 130.44, 130.50, 130.86, 131.51, 133.21, 138.04, 139.70, 158.97, 159.20, 167.87, 169.99. HRMS (FAB) calcd for $\mathrm{C}_{33} \mathrm{H}_{32} \mathrm{~N}_{2} \mathrm{O}_{3} \mathrm{ClS}[\mathrm{M}+\mathrm{H}]^{+}:$571.1822. Found: $571.1802(-3.6 \mathrm{ppm},-2.0 \mathrm{mmu})$. HPLC $\mathrm{t}_{\mathrm{r}} 41.6 \mathrm{~min}(S) ; \mathrm{t}_{\mathrm{r}} 46.9 \mathrm{~min}(R)$ [Chiralcel OD $(0.46 \mathrm{~cm} \times$ $25 \mathrm{~cm}$ ) (from Daicel Chemical Ind., Ltd.) Hexane $/ i-\mathrm{PrOH}, 99 / 1,1.0 \mathrm{~mL} / \mathrm{min},>98 \%$ ee.

\section{Enantioselective deprotonation/trapping of $N$-DAM 1,4-benzodiazepin-2-ones General procedure for reactions of $1 \mathrm{~d}$ at $-78^{\circ} \mathrm{C}$}

At $-78^{\circ} \mathrm{C}$ under nitrogen, to a stirred solution of $(S)-1 \mathbf{d}(102.0 \mathrm{mg}, 0.2 \mathrm{mmol}, 1.0$ equiv) and HMPA (208.4 $\mu \mathrm{L} 1.2 \mathrm{mmol}, 6.0$ equiv) in anhydrous THF (4.0 mL) was added KHMDS (1.0 $\mathrm{mL}, 0.5 \mathrm{mmol}, 2.5$ equiv, $0.5 \mathrm{M}$ in toluene). The resulting solution was stirred for a further 30.0 min before electrophile (2.0 to 10.0 equiv) was added dropwise via syringe. The reaction was stirred at $-78^{\circ} \mathrm{C}$ until the starting benzodiazepine was consumed (TLC). The reaction was quenched at $-78{ }^{\circ} \mathrm{C}$ with sat. $\mathrm{NH}_{4} \mathrm{Cl}(\mathrm{aq})(2.0 \mathrm{~mL})$, and extracted with $\mathrm{CH}_{2} \mathrm{Cl}_{2}(3 \times 10 \mathrm{~mL})$. The combined organic extracts were dried over anhydrous $\mathrm{Na}_{2} \mathrm{SO}_{4}$, filtered, and concentrated under reduced pressure. The crude product was purified by flash column chromatography on silica gel.<smiles>CON1C(=O)C(Br)(Br)N=C(c2ccccc2)c2cc(Cl)ccc21</smiles>

(3R)-(-)-1-di(p-anisyl)methyl-3-benzyl-7-chloro-1,3-dihydro-3-methyl-5-phenyl-2H-1,4benzodiazepin-2-one $[(R)-(-)-2 d]$ 
The general procedure was followed: $(S)-(+)-1 d$ (102 mg, $0.2 \mathrm{mmol})$, HMPA (208 $\mu \mathrm{L}, 1.2$ mmol), KHMDS (1.0 mL, $0.5 \mathrm{mmol}, 0.5 \mathrm{M}$ in toluene) and benzyl bromide (240 $\mu \mathrm{L}, 2.0 \mathrm{mmol})$. The reaction mixture was stirred at $-78^{\circ} \mathrm{C}$ for $4.0 \mathrm{~h}$. Purification with flash column chromatography on silica gel (1:4 Hexanes/EtOAc) provided $96 \mathrm{mg}(80 \%)$ of (-)-2d as white foam. The (3R)-stereochemistry was determined by hydrolysis to $(R)-(-)$ - $\alpha$-Me-Phe-OH 26 (see below $) .[\alpha]_{\mathrm{D}}{ }^{21}=-49.2\left(\mathrm{c}=3.0, \mathrm{CHCl}_{3}\right){ }^{1} \mathrm{H} \mathrm{NMR}\left(\mathrm{CDCl}_{3}\right)$ indicated a 64:36 ratio of axial-Me and equatorial-Me conformers: $\delta$ 7.61-7.57 (m, $1 \mathrm{H}+1 \mathrm{H}$ x 0.36 equatorial-Me), 7.49-7.44 $(\mathrm{m}, 1 \mathrm{H})$, 7.42-7.27 (m, $4 \mathrm{H}+1 \mathrm{H} \times 0.36$ equatorial-Me), 7.26-7.23 (m, 3H), 7.21-7.09 (m, 3H), 7.07 (dd, $J$ $=8.9 \mathrm{~Hz}, 2.5 \mathrm{~Hz}, 2 \mathrm{H}$ x 0.64 axial-Me), 7.01-6.95 (m, $3 \mathrm{H}+2 \mathrm{H}$ x 0.64 axial-Me), 6.94-6.90 (m, $1 \mathrm{H}), 6.89-6.85(\mathrm{~m}, 2 \mathrm{H}$ x 0.36 equatorial-Me), 6.71-6.66 (m, 2H), 3.86 (s, 3H x 0.64 axial-Me), $3.84(\mathrm{~s}, 3 \mathrm{H}$ x 0.36 equatorial-Me), $3.83(\mathrm{~d}, J=13.7 \mathrm{~Hz}, 3 \mathrm{H}$ x 0.64 axial), 3.78 (s, $3 \mathrm{H}$ x 0.36 equatorial-Me), 3.77 (1H x 0.64 axial-Me), 3.31 (d, $J=13.2 \mathrm{~Hz}, 1 \mathrm{H}$ x 0.64 axial-Me), 2.79 (d, $J$ $=14.2 \mathrm{~Hz}, 1 \mathrm{H}$ x 0.36 equatorial-Me), $2.62(\mathrm{~d}, J=14.2 \mathrm{~Hz}, 1 \mathrm{H} \times 0.36$ equatorial-Me $), 1.89(\mathrm{~s}, 3 \mathrm{H}$ x 0.36 equatorial-Me), 0.92 (s, 3H x 0.64 axial-Me). ${ }^{13} \mathrm{C} \mathrm{NMR}\left(\mathrm{CDCl}_{3}\right): \delta 173.3,172.7,165.8$, $165.4,159.0,158.9,158.8,158.7,139.8,139.7,139.6,139.5,138.5,136.8,134.6,134.5,132.3$, $131.5,131.4,130.7,130.64,130.60,130.5,130.4,130.3,130.2,130.1,130.0,129.9,129.6,129.5$, 129.4, 129.0, 128.9, 128.4, 128.3, 128.2, 127.6, 126.8, 126.3, 125.5, 114.0, 113.9, 113.7, 113.6, 68.5, 66.3, 66.2, 65.4, 55.5, 55.4, 55.3, 47.7, 38.8, 29.3, 17.7. HRMS calcd. for $\mathrm{C}_{38} \mathrm{H}_{34} \mathrm{ClN}_{2} \mathrm{O}_{3}$ $(\mathrm{M}+1)$ 601.2258, found $601.2257(-0.1 \mathrm{ppm},-0.1 \mathrm{mmu})$. HPLC $\mathrm{t}_{\mathrm{r}} 10.9 \mathrm{~min}(S) ; \mathrm{t}_{\mathrm{r}} 13.1 \mathrm{~min}(R)$ [Chiralpak AD (0.46 cm x $25 \mathrm{~cm}$ ) (from Daicel Chemical Ind., Ltd.) Hexane/i-PrOH , 92/8, 1.0 $\mathrm{mL} / \mathrm{min}, 99 \%$ ee.

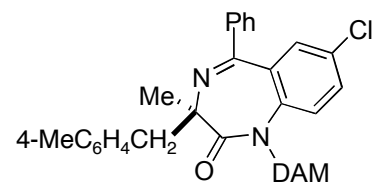

(3R)-(-)-1-di(p-anisyl)methyl-7-chloro-1,3-dihydro-3-methyl-3-(4-methylbenzyl)-5-phenyl2H-1,4-benzodiazepin-2-one [(R)-(-)-9d]

The general procedure was followed with $(S)-(+)-1 d(102 \mathrm{mg}, 0.2 \mathrm{mmol})$, HMPA (208 $\mu \mathrm{L}$, $1.2 \mathrm{mmol})$, KHMDS (1.0 mL, $0.5 \mathrm{mmol}, 0.5 \mathrm{M}$ in toluene) and 4-methyl benzyl bromide (370 $\mathrm{mg}$, $2.0 \mathrm{mmol}$ ). The reaction mixture was stirred at $-78{ }^{\circ} \mathrm{C}$ for $6.0 \mathrm{~h}$. Purification with flash column chromatography on silica gel (1:4 Hexanes/EtOAc) provided $101 \mathrm{mg}(82 \%)$ of (-)-9d as white foam. The $(R)$-stereochemistry was determined by hydrolysis to $(R)-(-)-\alpha-\mathrm{Me}-4-\mathrm{Me}-\mathrm{Phe}-\mathrm{OH} 27$ (see below). $[\alpha]_{\mathrm{D}}{ }^{21}=-34.0\left(\mathrm{c}=3.2, \mathrm{CHCl}_{3}\right){ }^{1} \mathrm{H} \mathrm{NMR}\left(\mathrm{CDCl}_{3}\right)$ indicated a 61:39 ratio of axialMe and equatorial-Me conformers: $\delta 7.50-7.43(\mathrm{~m}, 2 \mathrm{H}), 7.42-7.29(\mathrm{~m}, 4 \mathrm{H}), 7.28-7.22(\mathrm{~m}, 2 \mathrm{H})$, 7.20-7.12 (m, $2 \mathrm{H}+1 \mathrm{H} \times 0.39$ equatorial-Me), 7.12-7.03 (m, 3H), 7.01-6.93 (m, $4 \mathrm{H}+1 \mathrm{H} \times 0.61$ axial-Me), 6.89 (d, $J=2.3 \mathrm{~Hz}, 4 \mathrm{H}$ x 0.61 axial-Me), 6.88-6.84 (AA'XX' pattern, $2 \mathrm{H} \times 0.39$ equatorial-Me), $6.81(\mathrm{~d}, J=8.0 \mathrm{~Hz}, 2 \mathrm{H}$ x 0.39 equatorial-Me), 6.70-6.65 (m, 2H), $3.85(\mathrm{~s}, 3 \mathrm{H} \mathrm{x}$ 0.61 axial-Me), 3.84 (s, $3 \mathrm{H}$ x 0.39 equatorial-Me), 3.80 (d, $J=13.5 \mathrm{~Hz}, 1 \mathrm{H} \times 0.61$ axial-Me), $3.27(\mathrm{~d}, J=13.5 \mathrm{~Hz}, 1 \mathrm{H}$ x 0.61 axial-Me), 2.75 (d, $J=14.0 \mathrm{~Hz}, 1 \mathrm{H}$ x 0.39 equatorial-Me), 2.56 (d, $J=14.2 \mathrm{~Hz}, 1 \mathrm{H} \times 0.39$ equatorial-Me), 2.37 (s, $3 \mathrm{H}$ x 0.61 equatorial-Me), 2.35 (s, $3 \mathrm{H} \times 0.39$ equatorial-Me), 1.89 (s, $3 \mathrm{H} \times 0.39$ equatorial-Me), 0.91 (s, $3 \mathrm{H} \times 0.61$ axial-Me). ${ }^{13} \mathrm{C} \mathrm{NMR}$ $\left(\mathrm{CDCl}_{3}\right): \delta 173.3,172.7,165.7,165.3,159.0,158.9,158.7,158.6,139.8,139.7,139.6,139.5$, $136.3,135.7,135.3,134.7,134.6,133.7,132.1,131.5,130.7,130.66,130.62$, 130.4, 130.2, 129.9, $129.6,129.5,129.4,129.0,128.9,128.86,128.82,128.3,128.2,126.3,125.5,114.0,113.8,113.6$, 68.6, 66.2, 65.4, 55.4, 55.39, 55.31, 47.2, 38.5, 29.4, 21.2, 17.7. HRMS calcd. for $\mathrm{C}_{39} \mathrm{H}_{36} \mathrm{ClN}_{2} \mathrm{O}_{3}$ 
$(\mathrm{M}+1)$ 615.2414, found $615.2424(+1.5 \mathrm{ppm},+0.9 \mathrm{mmu}) . \operatorname{HPLC~}_{\mathrm{r}} 30.8 \mathrm{~min}(S) ; \mathrm{t}_{\mathrm{r}} 41.3 \mathrm{~min}(R)$ [Chiralpak AD-H (0.46 cm x $25 \mathrm{~cm}$ ) (from Daicel Chemical Ind., Ltd.) Hexane/i-PrOH, 98/2, 1.0 $\mathrm{mL} / \mathrm{min}, 100 \%$ ee.

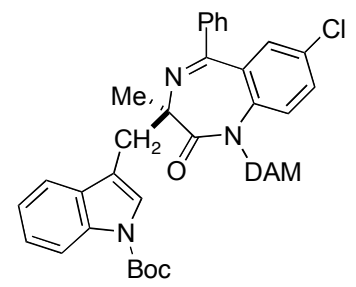

(3R)-(-)-1-di(p-anisyl)methyl-3-(N-tert-butoxycarbonyl-indol-3-yl)methyl-7-chloro-1,3dihydro-3-methyl-5-phenyl-2H-1,4-benzodiazepin-2-one [(R)-(-)-10d]

The general procedure was followed with $(S)-(+)-1 d(102 \mathrm{mg}, 0.2 \mathrm{mmol})$, HMPA $(208 \mu \mathrm{L}, 1.2$ mmol), KHMDS (1.0 mL, $0.5 \mathrm{mmol}, 0.5 \mathrm{M}$ in toluene) and 1-(tert-butyloxycarbonyl)-3(bromomethyl) indole $(124 \mathrm{mg}, 0.4 \mathrm{mmol}) .{ }^{9}$ The reaction mixture was stirred at $-78^{\circ} \mathrm{C}$ for $4 \mathrm{~h}$. Purification with flash column chromatography on silica gel (1:4 Hexane/EtOAc) provided 142 $\mathrm{mg}(96 \%)$ of $(-)-\mathbf{1 0 d}$ as colorless oil. The $(R)$-stereochemistry is assumed based on retentive substitution. $[\alpha]_{\mathrm{D}}{ }^{21}=-95.5\left(\mathrm{c}=3.9, \mathrm{CHCl}_{3}\right){ }^{1} \mathrm{H} \mathrm{NMR}\left(\mathrm{CDCl}_{3}\right)$ indicated a 80:20 ratio of axialMe and equatorial-Me conformers: $\delta 8.18(\mathrm{br}, 1 \mathrm{H}), 7.82-7.71(\mathrm{~m}, 2 \mathrm{H}), 7.51-7.42(\mathrm{~m}, 1 \mathrm{H}), 7.41$ $7.20(\mathrm{~m}, 7 \mathrm{H}), 7.19-7.02(\mathrm{~m}, 4 \mathrm{H}), 7.01-6.82(\mathrm{~m}, 5 \mathrm{H}), 6.73-6.65(\mathrm{~m}, 2 \mathrm{H}), 3.86(\mathrm{~s}, 3 \mathrm{H}), 3.83(\mathrm{~s}, 1 \mathrm{H}$ x 0.8 axial-Me), 3.80 (s, $1 \mathrm{H}$ x 0.2 equatorial-Me), 3.78 (s, $3 \mathrm{H}$ x 0.8 axial-Me), 3.76 (s, $3 \mathrm{H}$ x 0.2 equatorial-Me), 3.48 (d, $J=14.5 \mathrm{~Hz}, 1 \mathrm{H}$ x 0.8 axial-Me), 2.88-2.76 (m, $1 \mathrm{H}$ x 0.2 equatorial-Me), $2.02(\mathrm{~s}, 3 \mathrm{H} \times 0.2$ equatorial-Me), $1.71(\mathrm{~s}, 9 \mathrm{H} \times 0.8$ axial-Me), $1.63(\mathrm{~s}, 9 \mathrm{H} \times 0.2), 1.59(\mathrm{~s}, 0.3 \mathrm{H})$, 0.99 (s, 2.7H). ${ }^{13} \mathrm{C} \mathrm{NMR}\left(\mathrm{CDCl}_{3}\right): \delta 173.2,172.5,166.1,165.8,159.0,158.8,158.7,149.9,149.7$, 139.67, 139.66, 139.5, 139.4, 135.3, 134.5, 134.4, 132.8, 131.5 (partially resolved peak), 130.6, $130.5,130.4,130.3,130.0,129.9,129.7,129.6,129.5,129.0,128.9,128.8,128.4,128.2,126.2$, $125.6,125.5,124.5,124.1,124.0,122.4,122.3,120.4,119.1,117.1,115.4,115.1,115.0,114.0$, 113.8, 113.6, 83.6, 83.2, 68.4, 66.7, 66.0, 65.3, 55.4, 55.36, 55.32, 37.0, 29.9, 28.4, 28.2, 28.0, 17.8 HRMS calcd. for $\mathrm{C}_{45} \mathrm{H}_{43} \mathrm{ClN}_{3} \mathrm{O}_{5}(\mathrm{M}+1) 740.2891$, found $740.2892(+0.1 \mathrm{ppm},+0.1 \mathrm{mmu})$. HPLC $\mathrm{t}_{\mathrm{r}} 13.5$ min $((-)$-enantiomer, putatively $(R))$; $\mathrm{t}_{\mathrm{r}} 17.6$ min $((+)$-enantiomer, putatively $(S))$ [Chiralpak AD (0.46 cm x $25 \mathrm{~cm})$ ] (from Daicel Chemical Ind., Ltd.) Hexane/i-PrOH, 97/3, $1.0 \mathrm{~mL} / \mathrm{min}, 100 \%$ ee.<smiles>[CH]ON1C(=O)C(CC=C)N=C(c2ccccc2)c2cc(Cl)ccc21</smiles>

(3R)-(+)-3-allyl-1-di(p-anisyl)methyl-7-chloro-1,3-dihydro-3-methyl-5-phenyl-2H-1,4benzodiazepin-2-one $[(R)-(+)-11 d]$

The general procedure was followed with $(S)-(+)-1 d(102.0 \mathrm{mg}, 0.2 \mathrm{mmol})$, HMPA (208 $\mu \mathrm{L}, 1.2$ mmol), KHMDS (1.0 mL, $0.5 \mathrm{mmol}, 0.5 \mathrm{M}$ in toluene) and allyl bromide (174 $\mu \mathrm{L}, 2.0 \mathrm{mmol})$.

The reaction mixture was stirred at $-78^{\circ} \mathrm{C}$ for $4 \mathrm{~h}$. Purification with flash column chromatography on silica gel (1:4 Hexanes/EtOAc) provided $97 \mathrm{mg}(88 \%)$ of $(+)-\mathbf{1 1 d}$ as white foam. The $(R)$-stereochemistry is assigned based on hydrolysis to $(R)-(-)$ - $\alpha$-Me-aspartic acid 28. $[\alpha]_{\mathrm{D}}{ }^{21}=+1.7\left(\mathrm{c}=3.5, \mathrm{CHCl}_{3}\right){ }^{1} \mathrm{H} \mathrm{NMR}\left(\mathrm{CDCl}_{3}\right)$ indicated a $54: 46$ ratio of axial-Me and equatorial-Me conformers: $\delta$ 7.48-7.41 $(\mathrm{m}, 1 \mathrm{H}), 7.40-7.33(\mathrm{~m}, 3 \mathrm{H}), 7.32-7.28(\mathrm{~m}, 1 \mathrm{H})$, 7.27-7.24 $(\mathrm{m}, 1 \mathrm{H}), 7.23-7.18(\mathrm{~m}, 1 \mathrm{H}), 7.17-7.11(\mathrm{~m}, 1 \mathrm{H}), 7.10-7.06(\mathrm{~m}, 1 \mathrm{H}$ x 0.54 axial-Me), $7.04(\mathrm{~s}, 1 \mathrm{H} \mathrm{x}$ 
0.54 axial-Me), 7.03-6.98 (m, $1 \mathrm{H}+2 \mathrm{H}$ x 0.46 equatorial-Me), 6.98-6.88 (m, 4H), 6.68-6.64 (m, $2 \mathrm{H}), 6.35-6.21(\mathrm{~m}, 1 \mathrm{H} \times 0.54$ axial-Me), 5.68-5.79 (m, $1 \mathrm{H} \times 0.46$ equatorial), 5.28-5.18 (m, $1 \mathrm{H})$, $5.06(\mathrm{dd}, J=10.1 \mathrm{~Hz}, 1.8 \mathrm{~Hz}, 1 \mathrm{H} \times 0.46$ equatorial), 4.69 (dd, $J=17.0 \mathrm{~Hz}, 1.6 \mathrm{~Hz}, 1 \mathrm{H} \times 0.46$ equatorial-Me), 3.84 (s, 3H x 0.54 axial-Me), 3.83 (s, $3 \mathrm{H}$ x 0.46 equatorial-Me), 3.763 (s, $3 \mathrm{H} \mathrm{x}$ 0.46 equatorial-Me), $3.760(\mathrm{~s}, 3 \mathrm{H}$ x 0.56 axial-Me), 3.19 (dd, $J=13.9 \mathrm{~Hz}, 5.8 \mathrm{~Hz}, 1 \mathrm{H}$ x 0.56 axial$\mathrm{Me}), 2.81$ (dd, $J=13.7 \mathrm{~Hz}, 8.2 \mathrm{~Hz}, 1 \mathrm{H} \times 0.56$ axial-Me), $2.14-2.02(\mathrm{~m}, 1 \mathrm{H}), 1.92(\mathrm{~s}, 3 \mathrm{H}$ x 0.46 equatorial-Me), 0.98 (s, $3 \mathrm{H}$ x 0.54 axial-Me). ${ }^{13} \mathrm{C} \mathrm{NMR}\left(\mathrm{CDCl}_{3}\right): \delta 173.0,172.6,165.7,165.5$, 158.96, 158.93, 158.7, 139.6, 139.5(partially resolved peaks), 139.3, 135.7, 134.6, 134.4, 133.1, $131.5,131.4,130.6,130.5,130.46,130.40,130.3,130.0,129.7,129.5,129.46,129.44,129.0$, $128.9,128.8,128.3,126.5,126.3,118.6,118.2,113.9,113.8,113.6,67.6,65.9,65.7,65.3,55.4$, 55.3, 47.3, 37.3, 28.8, 18.1. HRMS calcd. for $\mathrm{C}_{34} \mathrm{H}_{32} \mathrm{ClN}_{2} \mathrm{O}_{3}(\mathrm{M}+1)$ 551.2101, found 551.2125 (+4.3 ppm, +2.4 mmu). HPLC $\mathrm{t}_{\mathrm{r}} 18.4 \mathrm{~min}((-)$-enantiomer, putatively $(S))$; $\mathrm{t}_{\mathrm{r}} 22.1 \mathrm{~min}((+)-$ enantiomer, putatively $(R))$ [Chiralcel OD $(0.46 \mathrm{~cm}$ x $25 \mathrm{~cm})$ (from Daicel Chemical Ind., Ltd.)] Hexane/i-PrOH, 99/1, $1.0 \mathrm{~mL} / \mathrm{min}, 100 \%$ ee.

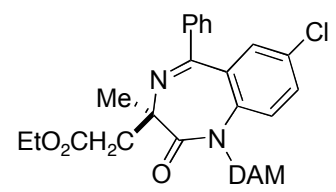

(3R)-(-)-1-di(p-anisyl)methyl-7-chloro-3-(ethoxycarbonyl)methyl-1,3-dihydro-3-methyl-5phenyl-2H-1,4-benzodiazepin-2-one [(R)-(-)-12d]

At $-78^{\circ} \mathrm{C}$ under nitrogen, to a stirred solution of $(S)-(+)-1 \mathbf{d}(102.0 \mathrm{mg}, 0.2 \mathrm{mmol}, 1.0$ equiv) and HMPA (208.4 $\mu \mathrm{L}, 1.2 \mathrm{mmol}, 6.0$ equiv) in anhydrous THF $(4.0 \mathrm{~mL})$ was added KHMDS (1.0 $\mathrm{mL}, 0.5 \mathrm{mmol}, 2.5$ equiv, $0.5 \mathrm{M}$ in toluene). The resulting solution was stirred for a further 30.0 min at $-78^{\circ} \mathrm{C}$ and then transferred via a cannula into a solution of ethyl bromoacetate $(223 \mu \mathrm{L}$ $2.0 \mathrm{mmol}, 10.0$ equiv) and HMPA $(104 \mu \mathrm{L}, 0.6 \mathrm{mmol})$ in dried $\mathrm{THF}(2.0 \mathrm{~mL})$ at $-78^{\circ} \mathrm{C}$. The reaction was stirred at $-78^{\circ} \mathrm{C}$ for $2.0 \mathrm{~h}$ at which the starting benzodiazepine $(S)-(+)$-1d was consumed (TLC). The reaction was quenched at $-78{ }^{\circ} \mathrm{C}$ with sat. $\mathrm{NH}_{4} \mathrm{Cl}(\mathrm{aq})(2.0 \mathrm{~mL})$, and extracted with $\mathrm{CH}_{2} \mathrm{Cl}_{2}(3 \times 10 \mathrm{~mL})$. The combined organic extracts were dried over anhydrous $\mathrm{Na}_{2} \mathrm{SO}_{4}$, filtered, and concentrated under reduced pressure. The crude product was purified by flash column chromatography on silica gel (1:3 Hexanes/EtOAc) to afford $102.7 \mathrm{mg}(86 \%)$ of $(R)-(-)-\mathbf{1 2 d}$ as colorless oil. $(R)$-Stereochemistry is assumed on the basis of retentive subsitution. $[\alpha]_{\mathrm{D}}{ }^{23}=-61.5\left(\mathrm{c}=3.4, \mathrm{CHCl}_{3}\right){ }^{1} \mathrm{H} \mathrm{NMR}\left(\mathrm{CDCl}_{3}\right)$ indicated a $67: 33$ ratio of axial-Me and equatorial-Me conformers: $\delta 7.48-7.41(\mathrm{~m}, 1 \mathrm{H}), 7.40-7.31(\mathrm{~m}, 2 \mathrm{H}+2 \mathrm{H}$ x 0.33 equatorial-Me), 7.30-7.19 (m, $4 \mathrm{H}+1 \mathrm{H} \times 0.33$ equatorial-Me), 7.18-7.07 (m, 2H), 7.04 (s, 2H x 0.33 equatorial$\mathrm{Me}), 7.01(\mathrm{~d}, J=2.5 \mathrm{~Hz}, 2 \mathrm{H} \times 0.33$ equatorial-Me), $6.99-6.89(\mathrm{~m}, 4 \mathrm{H}+1 \mathrm{H} \times 0.33$ equatorial-Me), 6.69-6.61 (m, 2H), 4.29-4.10 (m, 2H), $3.89(\mathrm{~s}, 3 \mathrm{H} \times 0.33$ equatorial-Me), $3.83(\mathrm{~s}, 3 \mathrm{H} \times 0.67$ axial-Me), 3.76 (s, $3 \mathrm{H} \times 0.33$ equatorial-Me), 3.75 (s, $3 \mathrm{H} \times 0.67$ axial-Me), 3.37 (d, $J=15.6 \mathrm{~Hz}$, $1 \mathrm{H} \times 0.67$ axial-Me), 3.19 (d, $J=15.6 \mathrm{~Hz}, 1 \mathrm{H}$ x 0.67 axial-Me), 2.32 (d, $J=14.6 \mathrm{~Hz}, 1 \mathrm{H}$ x 0.33 equatorial-Me), $2.18(\mathrm{~d}, J=14.6 \mathrm{~Hz}, 1 \mathrm{H} \mathrm{x} 0.33$ equatorial-Me), 1.94 (s, 3H x 0.33equatorial-Me), $1.31(\mathrm{t}, J=7.3 \mathrm{~Hz}, 3 \mathrm{H} \times 0.67 \mathrm{axial}), 1.29(\mathrm{~s}, 3 \mathrm{H} \times 0.67$ axial-Me), $1.23(\mathrm{t}, J=7.1 \mathrm{~Hz}, 3 \mathrm{H} \times 0.33$ equatorial-Me). ${ }^{13} \mathrm{C} \mathrm{NMR}\left(\mathrm{CDCl}_{3}\right): \delta 171.9,171.8,170.9,169.9,166.3,165.3,158.9,158.8$, $158.7,139.4,139.3,139.2,139.0,134.2,134.1,131.6,131.4,130.7,130.6,130.5,130.4,130.3$, 130.2 , 130.1, 129.8, 129.5, 129.1, 129.0, 128.86, 128.85, 128.3, 126.3, 125.7, 114.0, 113.8, 113.6, 65.8, 65.7, 65.4, 65.2, 60.9, 60.2, 55.4, 55.3, 47.3, 38.5, 28.9, 18.6, 14.4, 14.2. HRMS calcd. for $\mathrm{C}_{35} \mathrm{H}_{34} \mathrm{ClN}_{2} \mathrm{O}_{5}(\mathrm{M}+1)$ 597.2156, found 597.2202 (+7.7 ppm, +4.6 mmu). HPLC $\mathrm{t}_{\mathrm{r}} 11.0 \mathrm{~min}(S) ; \mathrm{t}_{\mathrm{r}}$ 
$13.1 \mathrm{~min}(R)$ [Chiralpak AD $(0.46 \mathrm{~cm} \times 25 \mathrm{~cm})$ (from Daicel Chemical Ind., Ltd.)] Hexane/i$\mathrm{PrOH}, 90 / 10,1.0 \mathrm{~mL} / \mathrm{min}, 99 \%$ ee.

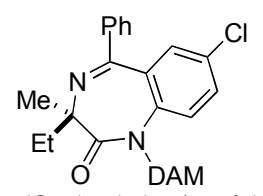

(3R)-(+)-1-di(p-anisyl)methyl-7-chloro-3-ethyl-1,3-dihydro-3-methyl-5-phenyl-2H-1,4benzodiazepin-2-one $[(R)-(+)-13 d]$

At $-78{ }^{\circ} \mathrm{C}$ under nitrogen, to a stirred solution of $(S)-(+)-1 \mathbf{d}(66.3 \mathrm{mg}, 0.13 \mathrm{mmol}, 1.0$ equiv) and HMPA $(138 \mu \mathrm{L}, 0.78 \mathrm{mmol}, 6.0$ equiv) in anhydrous THF $(2.0 \mathrm{~mL})$ was added LDA $(220.4 \mu \mathrm{L}$, $0.33 \mathrm{mmol}, 2.5$ equiv, $1.5 \mathrm{M}$ in hexane). The resulting solution was stirred for a further $30.0 \mathrm{~min}$ at $-78^{\circ} \mathrm{C}$ and then transferred via a cannula into a solution of ethyl iodide $(212 \mu \mathrm{L}, 2.6 \mathrm{mmol})$ and HMPA $(100 \mu \mathrm{L}, 0.57 \mathrm{mmol})$ in dried $\mathrm{THF}(1.0 \mathrm{~mL})$ at $-78^{\circ} \mathrm{C}$. The reaction was stirred at $78{ }^{\circ} \mathrm{C}$ for $5.0 \mathrm{~h}$ at which time the starting benzodiazepine $(S)-(+)-\mathbf{1 d}$ was consumed (TLC). The reaction was quenched at $-78{ }^{\circ} \mathrm{C}$ with sat. $\mathrm{NH}_{4} \mathrm{Cl}(\mathrm{aq})(2.0 \mathrm{~mL})$, and extracted with $\mathrm{CH}_{2} \mathrm{Cl}_{2}(3 \mathrm{x}$ $10 \mathrm{~mL}$ ). The combined organic extracts were dried over anhydrous $\mathrm{Na}_{2} \mathrm{SO}_{4}$, filtered, and concentrated under reduced pressure. The crude product was purified by flash column chromatography on silica gel (1:4 Hexanes / EtOAc) to afford $45.5 \mathrm{mg}(65 \%)$ of $(+)-\mathbf{1 3 d}$ as colorless oil. $(R)$-Stereochemistry is assumed based on retentive substitution. $[\alpha]_{\mathrm{D}}{ }^{23}=+15.0(\mathrm{c}=$ $\left.0.3, \mathrm{CHCl}_{3}\right){ }^{1} \mathrm{H} \mathrm{NMR}\left(\mathrm{CDCl}_{3}\right)$ indicated a 50:50 ratio of axial-Me and equatorial-Me conformers: $\delta$ 7.48-7.40 (m, $1 \mathrm{H}), 7.39-7.32(\mathrm{~m}, 3 \mathrm{H}), 7.31-7.27(\mathrm{~m}, 1 \mathrm{H}), 7.26-7.17(\mathrm{~m}, 1 \mathrm{H}+2 \mathrm{H} \times 0.50$ axialMe), $7.14(\mathrm{dd}, J=9.0 \mathrm{~Hz}, 2.1 \mathrm{~Hz}, 1 \mathrm{H}), 7.09(\mathrm{dt}, J=6.0 \mathrm{~Hz}, 2.3 \mathrm{~Hz}, 1 \mathrm{H}), 7.05-6.88(\mathrm{~m}, 6 \mathrm{H}), 6.71-$ $6.61(\mathrm{~m}, 2 \mathrm{H}), 3.83(\mathrm{~s}, 3 \mathrm{H}), 3.76(\mathrm{~d}, J=1.8 \mathrm{~Hz}, 3 \mathrm{H}), 2.41$ (sxt, $J=7.3 \mathrm{~Hz}, 1 \mathrm{H}$ x 0.50 axial-Me), 2.07 (sxt, $J=7.3 \mathrm{~Hz}, 1 \mathrm{H}$ x 0.5 axial-Me), $1.86(\mathrm{~s}, 3 \mathrm{H} \times 0.5$ equatorial-Me), $1.34-1.31(\mathrm{~m}, 1 \mathrm{H})$, $1.20(\mathrm{t}, J=7.3 \mathrm{~Hz}, 3 \mathrm{H}$ x 0.5 axial-Me), $0.98(\mathrm{~s}, 3 \mathrm{H} \times 0.5$ axial-Me), 0.79 (t, $J=7.3 \mathrm{~Hz}, 3 \mathrm{H} \times 0.5$ equatorial-Me). ${ }^{13} \mathrm{C} \mathrm{NMR}\left(\mathrm{CDCl}_{3}\right): \delta 173.5,173.3,165.5,165.4,158.9,158.8,158.7,139.7$, 139.6, 139.4, 134.4, 134.3, 131.4, 131.3, 130.8, 130.7, 130.5, 130.32, 130.30, 129.9, 129.6, $129.43,129.40,128.9,128.8,128.7,128.2,125.6,125.5,113.9,113.6,113.5,68.6,65.84,65.82$, $65.3,55.4,55.3,35.3,27.9,25.7,17.4,9.4,8.1$. HRMS calcd. for $\mathrm{C}_{33} \mathrm{H}_{32} \mathrm{ClN}_{2} \mathrm{O}_{3}(\mathrm{M}+1)$ 539.2101, found 539.2096 (-1.0 ppm, $-0.6 \mathrm{mmu})$. HPLC $\mathrm{t}_{\mathrm{r}} 26.1 \mathrm{~min}((+)$-enantiomer, putatively $(R)) ; \mathrm{t}_{\mathrm{r}} 31.4 \mathrm{~min}((-)$-enantiomer, putatively $(S))$ [Chiralpak AD ( $0.46 \mathrm{~cm} \times 25 \mathrm{~cm}$ ) (from Daicel Chemical Ind., Ltd.)] Hexane / i-PrOH, 98/2, $1.0 \mathrm{~mL} / \mathrm{min}, 96 \%$ ee.<smiles>CON1C(=O)[C@]2(N)N=C(c3ccccc3)c3cc(Cl)ccc3N12</smiles>

(3R)-(+)-1-di(p-anisyl)methyl-7-chloro-3-cyano-1,3-dihydro-3-methyl-5-phenyl-2H-1,4benzodiazepin-2-one $[(R)-(+)-14 d]$

The general procedure of (-)-12d was followed with $(S)-(+)-1 \mathbf{d}(102.0 \mathrm{mg}, 0.2 \mathrm{mmol})$, HMPA (208.4 $\mu \mathrm{L}, 1.2 \mathrm{mmol})$, KHMDS (1.0 mL, $0.5 \mathrm{mmol}, 0.5 \mathrm{M}$ in toluene) and $p$-toluenesulfonyl cyanide (72.6 mg, $0.4 \mathrm{mmol}, 2.0$ equiv). The reaction mixture was stirred at $-78{ }^{\circ} \mathrm{C}$ for $10 \mathrm{~min}$. Purification with flash column chromatography on silica gel (1:4 Hexanes/EtOAc) provided $101.7 \mathrm{mg}(95 \%)$ of $(+)-\mathbf{1 4 d}$ as white foam. $(R)$-Stereochemistry is assumed on the basis of retentive substitution. $[\alpha]_{\mathrm{D}}{ }^{20}=+169.2\left(\mathrm{c}=4.1, \mathrm{CHCl}_{3}\right) .{ }^{1} \mathrm{H} \mathrm{NMR}\left(\mathrm{CDCl}_{3}\right) \delta 7.60-7.49(\mathrm{~m}, 1 \mathrm{H})$, $7.48(\mathrm{~m}, 2 \mathrm{H}), 7.38-7.21(\mathrm{~m}, 6 \mathrm{H}), 7.11(\mathrm{~d}, J=2.3 \mathrm{~Hz}, 1 \mathrm{H}), 7.04(\mathrm{~m}, 5 \mathrm{H}), 6.74-6.62(\mathrm{~m}, 2 \mathrm{H}), 3.85$ 
(s, 3H), $3.76(\mathrm{~s}, 3 \mathrm{H}), 2.23(\mathrm{~s}, 3 \mathrm{H}) .{ }^{13} \mathrm{C}$ NMR $\left(\mathrm{CDCl}_{3}\right): \delta 170.4,164.4,159.3,159.1,138.7,137.3$, 132.7, 131.8, 131.7, 131.6, 131.4, 129.9, 129.5, 129.4, 129.3, 128.8, 128.6, 126.3, 116.2, 114.2, 113.8, 65.9, 58.4, 55.5, 55.3, 26.6 HRMS calcd. for $\mathrm{C}_{32} \mathrm{H}_{27} \mathrm{ClN}_{3} \mathrm{O}_{3}(\mathrm{M}+1)$ 536.1741, found $536.1736(-1.0 \mathrm{ppm},-0.5 \mathrm{mmu})$. HPLC $\mathrm{t}_{\mathrm{r}} 9.9 \mathrm{~min}((-)$-enantiomer, putatively $(S)) ; \mathrm{t}_{\mathrm{r}} 13.2 \mathrm{~min}$ $((+)$-enantiomer, putatively $(R))$ [Chiralpak $\mathrm{AD}(0.46 \mathrm{~cm} \times 25 \mathrm{~cm})$ (from Daicel Chemical Ind., Ltd.)] Hexane/i-PrOH , 90/10, $1.0 \mathrm{~mL} / \mathrm{min}, 99 \%$ ee.

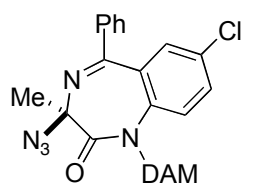

(3R)-(+)-1-di(p-anisyl)methyl-3-azido-7-chloro-1,3-dihydro-3-methyl-5-phenyl-2H-1,4benzodiazepin-2-one $[(R)-(+)-15 d]$

At $-78{ }^{\circ} \mathrm{C}$ under nitrogen, to a stirred solution of $(S)-(+)-1 \mathbf{d}(51.0 \mathrm{mg}, 0.1 \mathrm{mmol}, 0.1$ equiv) and HMPA (104 $\mu \mathrm{L} 0.6 \mathrm{mmol}, 6.0$ equiv) in anhydrous THF $(2.0 \mathrm{~mL})$ was added KHMDS $(0.5 \mathrm{~mL}$, $0.25 \mathrm{mmol}, 2.5$ equiv, $0.5 \mathrm{M}$ in toluene). The resulting solution was stirred for $30.0 \mathrm{~min}$ at -78 ${ }^{\circ} \mathrm{C}$ and followed by adding a solution of 2,4,6-triisopropylbenzenesulfonyl azide ${ }^{10}$ (77.4 mg, 0.25 $\mathrm{mmol})$ in dried THF $(0.5 \mathrm{~mL})$ to it. After stirring at $-78{ }^{\circ} \mathrm{C}$ for a further $30 \mathrm{~min}$, acetic acid $(0.1$ $\mathrm{mL}, 1.8 \mathrm{mmol}$ ) was added at $-78^{\circ} \mathrm{C}$ and the reaction warmed to r.t. for 2.0 hours. The reaction was quenched at r.t. with $10 \% \mathrm{NaHCO}_{3}$ and stirring for a another $30 \mathrm{~min}$. The reaction solution was extracted with $\mathrm{CH}_{2} \mathrm{Cl}_{2}(3 \times 10 \mathrm{~mL})$. The combined organic extracts were dried over anhydrous $\mathrm{Na}_{2} \mathrm{SO}_{4}$, filtered, and concentrated under reduced pressure. The crude product was purified by flash column chromatography on silica gel (1:4 Hexanes / EtOAc) to afford $48.6 \mathrm{mg}$ $(88 \%)$ of $(+)-\mathbf{1 5 d}$ as colorless oil. $(R)$-Stereochemistry is assumed based on retentive substitution. $[\alpha]_{\mathrm{D}}^{21}=+75.6\left(\mathrm{c}=2.0, \mathrm{CHCl}_{3}\right){ }^{1} \mathrm{H} \mathrm{NMR}\left(\mathrm{CDCl}_{3}\right)$ indicated a 30:70 ratio of axialMe and equatorial-Me conformers: $\delta 7.54-7.47(\mathrm{~m}, 1 \mathrm{H}), 7.44-7.33(\mathrm{~m}, 4 \mathrm{H}), 7.28-7.19(\mathrm{~m}, 2 \mathrm{H})$, 7.18-7.10 (m, $1 \mathrm{H}+1 \mathrm{H} \times 0.3$ axial-Me), 7.09-7.06 (m, 1H), 7.04 (s, $1 \mathrm{H} \times 0.7$ equatorial-Me), 6.98-6.90 (m, 4H), 6.70-6.62 (m, 2H), 3.84 (s, $3 \mathrm{H}$ x 0.3 axial-Me), 3.83 (s, $3 \mathrm{H} \mathrm{x} 0.7$ equatorial$\mathrm{Me}), 3.76$ (s, $3 \mathrm{H}), 2.18$ (s, $3 \mathrm{H}$ x 0.7 equatorial-Me), 1.14 (s, $3 \mathrm{H}$ x 0.3 axial-Me). ${ }^{13} \mathrm{C} \mathrm{NMR}$ $\left(\mathrm{CDCl}_{3}\right): \delta 169.5,168.4,167.0,164.9,159.2,159.1,158.93,158.90,139.4,138.8,138.3,137.9$, $133.8,133.6,131.4,131.3,131.2,130.7,130.6,130.4,130.1,130.0,129.9,129.8,129.7,129.6$, $129.2,128.9,128.8,128.7,128.5,128.4,126.0,124.8,114.0,113.7,113.6,84.4,79.4,65.7,65.6$, 55.4, 55.3, 25.8, 19.4. HRMS calcd. for $\mathrm{C}_{31} \mathrm{H}_{26} \mathrm{ClN}_{5} \mathrm{O}_{3}(\mathrm{M}+1)$ 552.1802, found $552.1801(-0.2$ ppm, -0.1 mmu). HPLC $\mathrm{t}_{\mathrm{r}} 8.4 \mathrm{~min}((-)$-enantiomer, putatively $(S))$; $\mathrm{t}_{\mathrm{r}} 10.7 \mathrm{~min}((+)$-enantiomer, putatively $(R))$ [Chiralpak AD $(0.46 \mathrm{~cm} \times 25 \mathrm{~cm})$ (from Daicel Chemical Ind., Ltd.)] Hexane/i$\mathrm{PrOH}, 92 / 8,1.0 \mathrm{~mL} / \mathrm{min}, 100 \%$ ee.

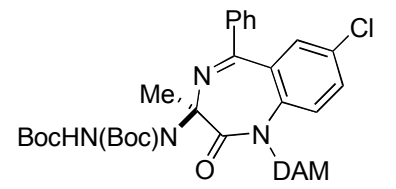

(3R)-(-)-1-di(p-anisyl)methyl-3-( $N, N^{\prime}$-di(tert-butoxycarbonyl)hydrazino-7-chloro-1,3dihydro-3-methyl-5-phenyl-2H-1,4-benzodiazepin-2-one $[(\boldsymbol{R})-(-)-16 \mathrm{~d}]$

The general procedure was followed with $(S)-(+)-1 \mathbf{d}(102.0 \mathrm{mg}, 0.2 \mathrm{mmol})$, HMPA $(208.4 \mu \mathrm{L}$, $1.2 \mathrm{mmol})$, KHMDS (1.0 mL, $0.5 \mathrm{mmol}, 0.5 \mathrm{M}$ in toluene) and di-tert-butyl-azodicarboxylate $(230.2 \mathrm{mg}, 1.0 \mathrm{mmol})$. The reaction mixture was stirred at $-78{ }^{\circ} \mathrm{C}$ for $10 \mathrm{~min}$. Purification with flash column chromatography on silica gel (1:4 Hexane / EtOAc) provided $139.2 \mathrm{mg}$ (94\%) of (- 
)-16d as white foam, $[\alpha]_{\mathrm{D}}{ }^{21}=-44.4\left(\mathrm{c}=3.3, \mathrm{CHCl}_{3}\right) .{ }^{1} \mathrm{H} \mathrm{NMR}\left(\mathrm{CDCl}_{3}\right)$ indicated a complex mixtures of conformers: $\delta$ 7.61-7.40 $(\mathrm{m}, 2 \mathrm{H}), 7.39-6.99(\mathrm{~m}, 13 \mathrm{H}), 6.70-6.51(\mathrm{~m}, 2 \mathrm{H}), 5.44(\mathrm{br}$, $0.03 \mathrm{H}), 5.33(\mathrm{br}, 0.09 \mathrm{H}), 5.14(\mathrm{br}, 0.05 \mathrm{H}), 4.67(\mathrm{~s}, 0.36 \mathrm{H}), 4.52(\mathrm{~s}, 0.28 \mathrm{H}), 3.86-3.81(\mathrm{~m}, 3 \mathrm{H})$, 3.77-3.72 (m, 3H), 2.29-2.10(m, 3H), 1.62-1.13(m, 18H). ${ }^{13} \mathrm{C} \mathrm{NMR}\left(\mathrm{CDCl}_{3}\right): \delta 172.4,167.9$, $167.8,167.7,167.5,164.9,164.6,159.1,158.8,158.7,154.7,154.6,153.2,152.8,152.5,150.3$, $139.8,139.4,138.5,138.1,137.9,134.3,131.7,131.6,131.5,131.2,131.1,130.8,130.4,130.3$, $130.2,130.0,129.9,129.5,129.0,128.2,128.1,127.1,126.9,125.6,125.5,123.3,113.9,113.7$, 113.6, 83.8, 83.6, 82.7, 82.5, 82.3, 82.1, 81.7, 81.1, 80.9, 65.8, 65.6, 65.3, 55.4, 55.3, 55.2, 28.5, 28.4, 28.2, 28.1, 27.8, 25.6, 24.9, 24.4, 24.3. HRMS calcd. for $\mathrm{C}_{41} \mathrm{H}_{46} \mathrm{ClN}_{4} \mathrm{O}_{7}(\mathrm{M}+1)$ 741.3055, found $741.3054(-0.1 \mathrm{ppm},-0.1 \mathrm{mmu})$. HPLC $\mathrm{t}_{\mathrm{r}} 13.4 \mathrm{~min}((+)$-enantiomer, putatively $(S)) ; \mathrm{t}_{\mathrm{r}} 18.1$ min ((-)-enantiomer, putatively $(R))$ [Chiralpak AD $(0.46 \mathrm{~cm} \times 25 \mathrm{~cm})$ (from Daicel Chemical Ind., Ltd.)] Hexane/i-PrOH , 90/10, $1.0 \mathrm{~mL} / \mathrm{min}, 100 \%$ ee.

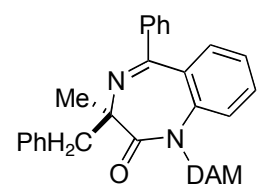

\section{(3R)-(-)-1-di(p-anisyl)methyl-3-benzyl-1,3-dihydro-3-methyl-5-phenyl-2H-1,4- benzodiazepin-2-one [(R)-(-)-17d]}

At $-42{ }^{\circ} \mathrm{C}$ under nitrogen, to a stirred solution of $(S)-5 \mathbf{d}(50.0 \mathrm{mg}, 0.105 \mathrm{mmol}, 1.0$ equiv) and HMPA (110 $\mu \mathrm{L}, 0.630 \mathrm{mmol}, 6.0$ equiv) in anhydrous DME $(3.1 \mathrm{~mL})$ was added KHMDS $(0.840 \mathrm{~mL}, 0.420 \mathrm{mmol}, 4.0$ equiv, $0.5 \mathrm{M}$ in toluene). The resulting solution was stirred for a further $30.0 \mathrm{~min}$ at $-42^{\circ} \mathrm{C}$ and then transferred via a cannula into a solution of benzyl bromide (126 $\mu \mathrm{L}, 1.05 \mathrm{mmol}, 10.0$ equiv) and HMPA $(55 \mu \mathrm{L}, 0.315 \mathrm{mmol})$ in dried DME $(1.6 \mathrm{~mL})$ at -42 ${ }^{\circ} \mathrm{C}$. The reaction was stirred at $-42^{\circ} \mathrm{C}$ for $1.5 \mathrm{~h}$ at which the starting benzodiazepine $(S)-5 \mathbf{d}$ was consumed (TLC, 1:3 EtOAC/hexanes). The reaction was quenched at $-42{ }^{\circ} \mathrm{C}$ with sat. $\mathrm{NH}_{4} \mathrm{Cl}$ (aq) $(5.0 \mathrm{~mL})$ and extracted with $\mathrm{CH}_{2} \mathrm{Cl}_{2}(3 \times 5 \mathrm{~mL})$. The combined organic extracts were dried over anhydrous $\mathrm{Na}_{2} \mathrm{SO}_{4}$, filtered, and concentrated under reduced pressure. The crude product was purified by flash chromatography on silica gel (1:3 EtOAc/hexanes) to afford $46.5 \mathrm{mg}$ $(78 \%)$ of $(R)-(-)-17 d$ as white foam. $(R)$-Stereochemistry of (-)-17d deduced based on similar sign of rotation compared to chloro-substituted analog (-)-2d. $[\alpha]_{\mathrm{D}}{ }^{25}=-10.8\left(c=0.023, \mathrm{CHCl}_{3}\right)$. ${ }^{1} \mathrm{H}$ NMR $\left(\mathrm{CDCl}_{3}\right)$ indicated a 60:40 mixture of axial-methyl and equatorial-methyl conformers $\delta$ $0.87\left(\mathrm{~s}, 3 \mathrm{H} \times 0.60 \mathrm{ax}\right.$-methyl), $1.87(\mathrm{~s}, 3 \mathrm{H} \times 0.40$ eq-methyl $), 2.59\left(\mathrm{~d},{ }^{2} J_{\mathrm{HH}}=14.0 \mathrm{~Hz}, 1 \mathrm{H} \times 0.40\right.$ eq-methyl), $2.75\left(\mathrm{~d},{ }^{2} J_{\mathrm{HH}}=13.5 \mathrm{~Hz}, 1 \mathrm{H} \times 0.40\right.$ eq-methyl $), 3.31\left(\mathrm{~d},{ }^{2} J_{\mathrm{HH}}=13.5 \mathrm{~Hz}, 1 \mathrm{H} \times 0.60\right.$ ax-methyl), $3.71\left(\mathrm{~d},{ }^{2} J_{\mathrm{HH}}=11 \mathrm{~Hz}, 1 \mathrm{H} \times 0.60 \mathrm{ax}-\right.$ methyl $), 3.74(\mathrm{~s}, 3 \mathrm{H} \times 0.60$, one conformer $)$, $3.75(\mathrm{~s}, 3 \mathrm{H} \times 0.40$, one conformer $), 3.83(\mathrm{~s}, 3 \mathrm{H} \times 0.40$, one conformer $), 3.85(\mathrm{~s}, 3 \mathrm{H} \times 0.60$, one conformer), $6.67\left(\mathrm{t},{ }^{3} J_{\mathrm{HH}}=7.5 \mathrm{~Hz}, 2 \mathrm{H}\right), 6.86-6.97(\mathrm{~m}, 5 \mathrm{H}), 7.01\left(\mathrm{~d},{ }^{3} J_{\mathrm{HH}}=7.0 \mathrm{~Hz}, 2 \mathrm{H}\right), 7.09-7.16$ $(\mathrm{m}, 2 \mathrm{H}), 7.20-7.29(\mathrm{~m}, 5 \mathrm{H}), 7.32-7.36(\mathrm{~m}, 5 \mathrm{H}), 7.42-7.44(\mathrm{~m}, 1 \mathrm{H}), 7.60\left(\mathrm{~d},{ }^{3} J_{\mathrm{HH}}=7.5 \mathrm{~Hz}, 1 \mathrm{H}\right)$. ${ }^{13} \mathrm{C} \mathrm{NMR}\left(\mathrm{CDCl}_{3}\right)$ was consistent with a 60:40 mixture of axial-methyl and equatorial-methyl conformers ( 57 resonances found for a possible $2 \times 38$ unique carbons): $\delta 17.51,29.16,38.33$, $47.74,55.31,55.41,55.44,65.64,66.10,66.59,68.36,113.45,113.48,113.57,113.75,113.90$, $124.07,124.14,124.69,124.85,126.19,126.65,127.55,128.09,128.13,128.21,128.98 .129 .09$, 129.42 , 129.61, 129.73, 129.96, 130.12, 130.19, 130.35, 130.84, 130.98, 131.01, 131.22, 131.51, 132.32, 133.12, 133.18, 137.09, 138.86, 140.39, 140.49, 141.07, 141.19, 158.57, 158.64, 158.80, 158.88, 166.69, 167.24, 172.96, 173.61. HRMS (FAB) calcd for $\mathrm{C}_{38} \mathrm{H}_{35} \mathrm{~N}_{2} \mathrm{O}_{3}[\mathrm{M}+\mathrm{H}]^{+}$: 567.2648. Found: $567.2646(-0.3 \mathrm{ppm},-0.2 \mathrm{mmu})$. HPLC $\mathrm{t}_{\mathrm{r}} 16.3 \mathrm{~min}(R) ; \mathrm{t}_{\mathrm{r}} 19.7 \mathrm{~min}(S)$ 
[Chiralcel OD $(0.46 \mathrm{~cm} \times 25 \mathrm{~cm})$ (from Daicel Chemical Ind., Ltd.) Hexane $/ i-\mathrm{PrOH}, 97 / 3,1.0$ $\mathrm{mL} / \mathrm{min}, 98 \%$ ee.<smiles>CON1C(=O)N(Cc2ccccc2)C(c2ccccc2)=NC(c2ccccc2)c2ccccc21</smiles>

(3S)-(+)-1-di(p-anisyl)methyl-3-benzyl-7-chloro-1,3-dihydro-3-methyl-5-phenyl-2H-1,4benzodiazepin-2-one $[(S)-(+)-2 d]$

At $-42{ }^{\circ} \mathrm{C}$ under nitrogen, to a stirred solution of $(S)-6 \mathbf{d}(50.0 \mathrm{mg}, 0.085 \mathrm{mmol}, 1.0$ equiv) and HMPA (90 $\mu \mathrm{L}, 0.512 \mathrm{mmol}, 6.0$ equiv) in anhydrous DME (2.4 mL) was added KHMDS (0.682 $\mathrm{mL}, 0.341 \mathrm{mmol}, 4.0$ equiv, $0.5 \mathrm{M}$ in toluene). The resulting solution was stirred for a further $30.0 \mathrm{~min}$ at $-42^{\circ} \mathrm{C}$ and then transferred via a cannula into a solution of methyl iodide $(53 \mu \mathrm{L}$, $0.853 \mathrm{mmol}, 10.0$ equiv) and HMPA $(45 \mu \mathrm{L}, 0.256 \mathrm{mmol})$ in dried DME $(1.2 \mathrm{~mL})$ at $-42^{\circ} \mathrm{C}$. The reaction was stirred at $-42^{\circ} \mathrm{C}$ for $1.4 \mathrm{~h}$ at which the starting benzodiazepine $(S)$-6d was consumed (TLC, 1:3 EtOAC/hexanes). The reaction was quenched at $-42{ }^{\circ} \mathrm{C}$ with sat. $\mathrm{NH}_{4} \mathrm{Cl}$ (aq) $(5.0 \mathrm{~mL})$ and extracted with $\mathrm{CH}_{2} \mathrm{Cl}_{2}(3 \times 5 \mathrm{~mL})$. The combined organic extracts were dried over anhydrous $\mathrm{Na}_{2} \mathrm{SO}_{4}$, filtered, and concentrated under reduced pressure. The crude product was purified by flash chromatography on silica gel (1:3 EtOAc/hexanes) to afford $40.5 \mathrm{mg}$ $(79 \%)$ of $(S)-(+)-2 d$ as white foam. $[\alpha]_{\mathrm{D}}^{25}=+39.6\left(c=2.03, \mathrm{CHCl}_{3}\right)$. HPLC $\mathrm{t}_{\mathrm{r}} 22.1 \mathrm{~min}(S)$; $\mathrm{t}_{\mathrm{r}}$ $24.7 \mathrm{~min}(R)$ [Chiralcel OD $(0.46 \mathrm{~cm} \times 25 \mathrm{~cm})$ (from Daicel Chemical Ind., Ltd.) Hexane $/ i-$ PrOH, 99/1, $1.0 \mathrm{~mL} / \mathrm{min},>99.5 \%$ ee. The ${ }^{1} \mathrm{H}$ NMR spectrum was identical to $(R)-(-)-2 d$.

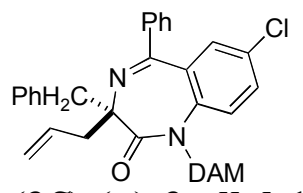

(3S)-(+)-3-allyl-1-di(p-anisyl)methyl-3-benzyl-7-chloro-1,3-dihydro-5-phenyl-2H-1,4benzodiazepin-2-one $[(S)-(+)-18 \mathrm{~d}]$

At $-42{ }^{\circ} \mathrm{C}$ under nitrogen, to a stirred solution of $(S)-6 \mathbf{d}(50.0 \mathrm{mg}, 0.085 \mathrm{mmol}, 1.0$ equiv) and HMPA ( $90 \mu \mathrm{L}, 0.512 \mathrm{mmol}, 6.0$ equiv) in anhydrous DME $(2.4 \mathrm{~mL})$ was added KHMDS (0.682 $\mathrm{mL}, 0.341 \mathrm{mmol}, 4.0$ equiv, $0.5 \mathrm{M}$ in toluene). The resulting solution was stirred for a further $30.0 \mathrm{~min}$ at $-42^{\circ} \mathrm{C}$ and then transferred via a cannula into a solution of allyl bromide $(74 \mu \mathrm{L}$, $0.850 \mathrm{mmol}, 10.0$ equiv) and HMPA $(45 \mu \mathrm{L}, 0.256 \mathrm{mmol})$ in dried DME $(1.2 \mathrm{~mL})$ at $-42{ }^{\circ} \mathrm{C}$. The reaction was stirred at $-42^{\circ} \mathrm{C}$ for $1.9 \mathrm{~h}$ at which the starting benzodiazepine $(S)-6 \mathrm{~d}$ was consumed (TLC, 1:3 EtOAC/hexanes). The reaction was quenched at $-42{ }^{\circ} \mathrm{C}$ with sat. $\mathrm{NH}_{4} \mathrm{Cl}$ (aq) $(5.0 \mathrm{~mL})$ and extracted with $\mathrm{CH}_{2} \mathrm{Cl}_{2}(3 \times 5 \mathrm{~mL})$. The combined organic extracts were dried over anhydrous $\mathrm{Na}_{2} \mathrm{SO}_{4}$, filtered, and concentrated under reduced pressure. The crude product was purified by flash chromatography on silica gel $\left(1^{\text {st }}\right.$ eluent $1: 3$ EtOAc/hexanes, $2^{\text {nd }}$ eluent $1: 4$ EtOAc/hexanes) to afford $30.9 \mathrm{mg}(58 \%)$ of $(R)-(+)-\mathbf{1 8 d}$ as clear yellow oil. $[\alpha]_{\mathrm{D}}{ }^{25}=+68.2(c=$ $\left.1.55, \mathrm{CHCl}_{3}\right)$. ${ }^{1} \mathrm{H} \mathrm{NMR}\left(\mathrm{CDCl}_{3}\right)$ indicated a $65: 35$ mixture of axial-allyl and equatorial-allyl conformers $\delta 1.80\left(\mathrm{dd},{ }^{2} J_{\mathrm{HH}}=14.8 \mathrm{~Hz},{ }^{3} J_{\mathrm{HH}}=8.4 \mathrm{~Hz}, 1 \mathrm{H} \times 0.65\right.$ ax-allyl $), 2.03\left(\mathrm{dd},{ }^{2} J_{\mathrm{HH}}=15.2\right.$ $\mathrm{Hz},{ }^{3} J_{\mathrm{HH}}=5.6 \mathrm{~Hz}, 1 \mathrm{H} \times 0.65 \mathrm{ax}$-allyl $), 2.46\left(\mathrm{~d},{ }^{2} J_{\mathrm{HH}}=14.4 \mathrm{~Hz}, 1 \mathrm{H} \times 0.35\right.$ eq-allyl $), 2.87\left(\mathrm{~d},{ }^{2} J_{\mathrm{HH}}\right.$ $=14.4 \mathrm{~Hz}, 1 \mathrm{H} \times 0.35$ eq-allyl $), 3.03\left(\mathrm{dd},{ }^{2} J_{\mathrm{HH}}=16.0 \mathrm{~Hz},{ }^{3} J_{\mathrm{HH}}=8.8 \mathrm{~Hz}, 1 \mathrm{H} \times 0.35\right.$ eq-allyl $), 3.22$ $\left(\mathrm{dd},{ }^{2} J_{\mathrm{HH}}=5.0 \mathrm{~Hz},{ }^{3} J_{\mathrm{HH}}=0.8 \mathrm{~Hz}, 1 \mathrm{H} \times 0.35\right.$ eq-allyl $), 3.53\left(\mathrm{~d},{ }^{2} J_{\mathrm{HH}}=14.0 \mathrm{~Hz}, 1 \mathrm{H} \times 0.65 \mathrm{ax}-\right.$ allyl), $3.75\left(\mathrm{~d},{ }^{2} J_{\mathrm{HH}}=8.4 \mathrm{~Hz}, 1 \mathrm{H} \times 0.65 \mathrm{ax}\right.$-allyl $), 3.76(\mathrm{~s}, 3 \mathrm{H} \times 0.35$ one conformer, overlapping with peak at 3.75$), 3.77(\mathrm{~s}, 3 \mathrm{H} \times 0.65$ one conformer $), 3.82(\mathrm{~s}, 3 \mathrm{H} \times 0.35$ one conformer $), 3.85$ 
(s, $3 \mathrm{H} \times 0.65$ one conformer), $4.69\left(\mathrm{~d},{ }^{2} J_{\mathrm{HH}}=16.0 \mathrm{~Hz}, 1 \mathrm{H} \times 0.65\right.$ ax-allyl $), 5.65\left(\mathrm{~d},{ }^{2} J_{\mathrm{HH}}=10.4\right.$ $\mathrm{Hz}, 1 \mathrm{H} \times 0.65$ ax-allyl), 5.27-5.31 (m, $2 \mathrm{H} \times 0.35$ eq-allyl), 5.85-5.91 (m, $1 \mathrm{H} \times 0.65$ ax-allyl), 6.31-6.42 (m, $1 \mathrm{H} \times 0.35$ eq-allyl), $6.65\left(\mathrm{t},{ }^{3} J_{\mathrm{HH}}=7.6 \mathrm{~Hz}, 2 \mathrm{H}\right), 6.79\left(\mathrm{~d},{ }^{3} J_{\mathrm{HH}}=8.4 \mathrm{~Hz}, 1 \mathrm{H}\right), 6.90-$ $6.97(\mathrm{~m}, 6 \mathrm{H}), 7.04(\mathrm{~s}, 1 \mathrm{H}), 7.08-7.18(\mathrm{~m}, 2 \mathrm{H}), 7.24-7.26(\mathrm{~m}, 1 \mathrm{H}), 7.28-7.40(\mathrm{~m}, 7 \mathrm{H}), 7.44-7.48$

$(\mathrm{m}, 1 \mathrm{H}), 7.61\left(\mathrm{~d},{ }^{3} J_{\mathrm{HH}}=6.8 \mathrm{~Hz}, 1 \mathrm{H}\right) .{ }^{13} \mathrm{C}$ NMR $\left(\mathrm{CDCl}_{3}\right)$ was consistent with a $65: 35$ mixture of axial-allyl and equatorial-allyl conformers (59 resonances found for a possible $2 \times 32$ unique carbons): $\delta 33.51,36.13,43.64,44.10,55.31,55.40,55.44,66.14,66.47,70.34,70.74,113.61$, $113.76,113.80,118.53,118.78,126.25,126.39,126.70,126.81,127.66,128.23,128.31,128.35$, $128.75,128.91,129.02,129.13,129.55,129.64,129.84,129.92$ (2 overlapping peaks), 130.12, $130.43,130.55,130.58,130.64,130.69,130.84,131.55,131.64,132.36,133.36,134.64,135.04$, $135.98,136.78,138.50,138.97,139.35,139.57,139.72,158.59,158.75,159.02,165.23,165.55$, 172.23, 172.48. HRMS (FAB) calcd for $\mathrm{C}_{40} \mathrm{H}_{36} \mathrm{~N}_{2} \mathrm{O}_{3} \mathrm{Cl}[\mathrm{M}+\mathrm{H}]^{+}$: 627.2414. Found: 627.2411 ($0.6 \mathrm{ppm},-0.4 \mathrm{mmu})$. HPLC $\mathrm{t}_{\mathrm{r}} 22.1 \mathrm{~min}(S) ; \mathrm{t}_{\mathrm{r}} 31.0 \mathrm{~min}(R)$ [Chiralpak AD $(0.46 \mathrm{~cm} \times 25 \mathrm{~cm})$ (from Daicel Chemical Ind., Ltd.) Hexane $/ \mathrm{i}-\mathrm{PrOH}, 97 / 3,1.0 \mathrm{~mL} / \mathrm{min}, 92 \%$ ee.

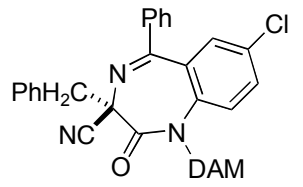

\section{(3R)-(+)-1-di(p-anisyl)methyl-3-benzyl-7-chloro-3-cyano-1,3-dihydro-5-phenyl-2H-1,4-} benzodiazepin-2-one $[(R)-(+)-19 d]$

At $-42^{\circ} \mathrm{C}$ under nitrogen, to a stirred solution of $(S)-6 \mathbf{d}(50.0 \mathrm{mg}, 0.085 \mathrm{mmol}, 1.0$ equiv) and HMPA ( $90 \mu \mathrm{L}, 0.512 \mathrm{mmol}, 6.0$ equiv) in anhydrous DME $(2.4 \mathrm{~mL})$ was added KHMDS $(0.682$ $\mathrm{mL}, 0.341 \mathrm{mmol}, 4.0$ equiv, $0.5 \mathrm{M}$ in toluene). The resulting solution was stirred for a further $30.0 \mathrm{~min}$ at $-42{ }^{\circ} \mathrm{C}$ and then transferred via a cannula into a solution of $p$-toluenesulfonyl cyanide (58.4 mg, $0.320 \mathrm{mmol}, 2.0$ equiv) and HMPA ( $45 \mu \mathrm{L}, 0.256 \mathrm{mmol})$ in dried DME $(1.2 \mathrm{~mL})$ at $42{ }^{\circ} \mathrm{C}$. The reaction was stirred at $-42^{\circ} \mathrm{C}$ for $47 \mathrm{~min}$ at which the starting benzodiazepine $(S)$-6d was consumed (TLC, $1: 20 \mathrm{Et}_{2} \mathrm{O} / \mathrm{DCM}$ ). The reaction was quenched at $-42{ }^{\circ} \mathrm{C}$ with sat. $\mathrm{NH}_{4} \mathrm{Cl}$ (aq) $(5.0 \mathrm{~mL})$ and extracted with $\mathrm{CH}_{2} \mathrm{Cl}_{2}(3 \times 5 \mathrm{~mL})$. The combined organic extracts were dried over anhydrous $\mathrm{Na}_{2} \mathrm{SO}_{4}$, filtered, and concentrated under reduced pressure. The crude product was purified by flash chromatography on silica gel $\left({ }^{\text {st }}\right.$ eluent $1: 20 \mathrm{Et}_{2} \mathrm{O} / \mathrm{DCM}, 2^{\text {nd }}$ eluent 1:3 EtOAc/hexanes) to afford $35.4 \mathrm{mg}(68 \%)$ of $(R)-(+)-19 d$ as clear yellow oil. $[\alpha]_{\mathrm{D}}{ }^{25}=+122(c=$ 1.77, $\left.\mathrm{CHCl}_{3}\right) .{ }^{1} \mathrm{H}$ NMR $\left(\mathrm{CDCl}_{3}\right) \delta 3.61\left(\mathrm{~d},{ }^{2} J_{\mathrm{HH}}=13.6 \mathrm{~Hz}, 1 \mathrm{H}\right), 3.76(\mathrm{~s}, 3 \mathrm{H}), 3.86(\mathrm{~s}, 3 \mathrm{H}), 4.05$ $\left(\mathrm{d},{ }^{2} J_{\mathrm{HH}}=14.0 \mathrm{~Hz}, 1 \mathrm{H}\right), 6.67\left(\mathrm{~d},{ }^{2} J_{\mathrm{HH}}=8.4 \mathrm{~Hz}, 2 \mathrm{H}\right), 6.92-6.99(\mathrm{~m}, 5 \mathrm{H}), 7.05(\mathrm{~s}, 1 \mathrm{H}), 7.20-7.34$ $(\mathrm{m}, 7 \mathrm{H}), 7.37-7.41(\mathrm{~m}, 4 \mathrm{H}), 7.51\left(\mathrm{t},{ }^{3} J_{\mathrm{HH}}=7.2 \mathrm{~Hz}, 1 \mathrm{H}\right), 7.60\left(\mathrm{~d},{ }^{3} J_{\mathrm{HH}}=7.6 \mathrm{~Hz}, 2 \mathrm{H}\right) .{ }^{13} \mathrm{C}$ NMR $\left(\mathrm{CDCl}_{3}\right)$ One conformer $\mathrm{CN}$ axial (29 resonances found for a possible 30 unique carbons): $\delta$ $43.75,55.34,55.48,63.30,65.97,113.85,114.24,114.64,126.18,127.62,128.12,128.52$, $128.86,129.36,129.44,129.55,129.99,131.42,131.52,131.74,131.82,132.78,134.54,137.51$, 138.82, 159.22, 159.34, 164.27, 170.20. HRMS (FAB) calcd for $\mathrm{C}_{38} \mathrm{H}_{31} \mathrm{~N}_{3} \mathrm{O}_{3} \mathrm{Cl}[\mathrm{M}+\mathrm{H}]^{+}$: 612.2054. Found: $612.1997(-9.3 \mathrm{ppm},-5.7 \mathrm{mmu}) . \quad H P L C \mathrm{t}_{\mathrm{r}} 26.5 \mathrm{~min}(R) ; \mathrm{t}_{\mathrm{r}} 32.7 \mathrm{~min}(S)$ [Chiralcel OD $(0.46 \mathrm{~cm} \times 25 \mathrm{~cm})$ (from Daicel Chemical Ind., Ltd.) Hexane/i-PrOH, 99/1, 1.0 $\mathrm{mL} / \mathrm{min}, 96 \%$ ee. 
<smiles>CCN1C(=O)C(Cc2ccccc2)N(OC)c2ccc(Cl)cc21</smiles>

(3R)-(-)-1-di(p-anisyl)methyl-3-benzyl-7-chloro-3-ethyl-1,3-dihydro-5-phenyl-2H-1,4benzodiazepin-2-one [(R)-(-)-20d]

At $-42{ }^{\circ} \mathrm{C}$ under nitrogen, to a stirred solution of $(S)-7 \mathbf{d}(200 \mathrm{mg}, 0.382 \mathrm{mmol}, 1.0$ equiv) and HMPA (398 $\mu \mathrm{L}, 2.29 \mathrm{mmol}, 6.0$ equiv) in anhydrous DME (11.0 mL) was added KHMDS (3.06 $\mathrm{mL}, 1.53 \mathrm{mmol}, 4.0$ equiv, $0.5 \mathrm{M}$ in toluene). The resulting solution was stirred for a further $30.0 \mathrm{~min}$ at $-42^{\circ} \mathrm{C}$ and then transferred via a cannula into a solution of benzyl bromide $(457 \mu \mathrm{L}$, $3.82 \mathrm{mmol}, 10.0$ equiv) and HMPA $(200 \mu \mathrm{L}, 1.15 \mathrm{mmol})$ in dried DME $(5.6 \mathrm{~mL})$ at $-42^{\circ} \mathrm{C}$. The reaction was stirred at $-42^{\circ} \mathrm{C}$ for $1.2 \mathrm{~h}$ at which the starting benzodiazepine $(S)-7 \mathbf{d}$ was consumed (TLC, 1:3 EtOAC/hexanes). The reaction was quenched at $-42{ }^{\circ} \mathrm{C}$ with sat. $\mathrm{NH}_{4} \mathrm{Cl}$ (aq) $(5.0 \mathrm{~mL})$ and extracted with $\mathrm{CH}_{2} \mathrm{Cl}_{2}(3 \times 5 \mathrm{~mL})$. The combined organic extracts were dried over anhydrous $\mathrm{Na}_{2} \mathrm{SO}_{4}$, filtered, and concentrated under reduced pressure. The crude product was purified by flash chromatography on silica gel (1:4 EtOAc/hexanes) to afford $152.0 \mathrm{mg}$ $(65 \%)$ of $(R)-(-)-20 d$ as clear yellow oil. $(R)$-Stereochemistry is deduced based on similar sign of rotation and HPLC elution order compared to Ala-derivative (-)-2d. $[\alpha]_{\mathrm{D}}{ }^{25}=-45.3(c=0.78$, $\left.\mathrm{CHCl}_{3}\right)$

${ }^{1} \mathrm{H} \mathrm{NMR}\left(\mathrm{CDCl}_{3}\right)$ indicated a 70:30 mixture of axial-Et and equatorial-Et conformers $\delta 0.87(\mathrm{t}$, $\left.{ }^{3} J_{\mathrm{HH}}=7.2 \mathrm{~Hz}, 3 \mathrm{H} \times 0.70 \mathrm{ax}-\mathrm{Et}\right), 1.10\left(\mathrm{q},{ }^{3} J_{\mathrm{HH}}=7.6 \mathrm{~Hz}, 1 \mathrm{H} \times 0.70 \mathrm{ax}-\mathrm{Et}\right), 1.20\left(\mathrm{q},{ }^{3} J_{\mathrm{HH}}=7.2 \mathrm{~Hz}\right.$, $1 \mathrm{H} \times 0.70 \mathrm{ax}-\mathrm{Et}), 1.26\left(\mathrm{t},{ }^{3} J_{\mathrm{HH}}=3.6 \mathrm{~Hz}, 3 \mathrm{H} \times 0.30 \mathrm{eq}-\mathrm{Et}\right.$, overlapping with peak at 1.20$), 2.20$ $\left(\mathrm{q},{ }^{3} J_{\mathrm{HH}}=6.8 \mathrm{~Hz}, 1 \mathrm{H} \times 0.30 \mathrm{eq}-\mathrm{Et}\right), 2.39\left(\mathrm{q},{ }^{3} J_{\mathrm{HH}}=6.8 \mathrm{~Hz}, 1 \mathrm{H} \times 0.30 \mathrm{eq}-\mathrm{Et}\right), 2.54\left(\mathrm{~d},{ }^{2} J_{\mathrm{HH}}=\right.$ $14.8 \mathrm{~Hz}, 1 \mathrm{H} \times 0.30 \mathrm{eq}-\mathrm{Et}), 2.75\left(\mathrm{~d},{ }^{2} J_{\mathrm{HH}}=14.4 \mathrm{~Hz}, 1 \mathrm{H} \times 0.30 \mathrm{eq}-\mathrm{Et}\right), 3.52\left(\mathrm{~d},{ }^{2} J_{\mathrm{HH}}=14.0 \mathrm{~Hz}\right.$, $1 \mathrm{H} \times 0.70 \mathrm{ax}-\mathrm{Et}), 3.68\left(\mathrm{~d},{ }^{2} J_{\mathrm{HH}}=14.4 \mathrm{~Hz}, 1 \mathrm{H} \times 0.70 \mathrm{ax}-\mathrm{Et}\right), 3.77(\mathrm{~s}, 3 \mathrm{H}), 3.82(\mathrm{~s}, 3 \mathrm{H} \times 0.30$ one conformer), $3.85(\mathrm{~s}, 3 \mathrm{H} \times 0.70$ one conformer $), 6.67\left(\mathrm{dd},{ }^{3} J_{\mathrm{HH}}=8.8 \mathrm{~Hz},{ }^{4} J_{\mathrm{HH}}=2.4 \mathrm{~Hz}, 2 \mathrm{H}\right), 6.82$ $\left(\mathrm{d},{ }^{3} J_{\mathrm{HH}}=8.8 \mathrm{~Hz}, 1 \mathrm{H}\right), 6.89\left(\mathrm{~d},{ }^{4} J_{\mathrm{HH}}=2.4 \mathrm{~Hz}, 1 \mathrm{H}\right), 6.95(\mathrm{~m}, 4 \mathrm{H}), 7.03\left(\mathrm{~d},{ }^{3} J_{\mathrm{HH}}=8.4 \mathrm{~Hz}, 1 \mathrm{H}\right)$, 7.06-7.10 (m, 2H), $7.16(\mathrm{~s}, 1 \mathrm{H} \times 0.70 \mathrm{ax}-\mathrm{Et}), 7.18(\mathrm{~s}, 1 \mathrm{H} \times 0.30 \mathrm{eq}-\mathrm{Et}), 7.24-7.32(\mathrm{~m}, 4 \mathrm{H}), 7.34-$ $7.40(\mathrm{~m}, 4 \mathrm{H}), 7.45\left(\mathrm{t},{ }^{3} J_{\mathrm{HH}}=7.2 \mathrm{~Hz}, 1 \mathrm{H}\right), 7.59\left(\mathrm{~d},{ }^{3} J_{\mathrm{HH}}=7.2 \mathrm{~Hz}, 1 \mathrm{H}\right) .{ }^{13} \mathrm{C} \mathrm{NMR}\left(\mathrm{CDCl}_{3}\right)$ was consistent with a 70:30 mixture of axial-Et and equatorial-Et conformers ( 53 resonances found for a possible $2 \times 31$ unique carbons): $\delta 8.44,10.02,21.66,31.81,35.23,43.03,55.31,55.43$, $66.21,66.63,70.71,71.70,113.65,113.74,113.96,125.81,126.25,126.37,126.72,127.61$, $128.18,128.29,128.33,128.62,128.90,128.97,129.07,129.52,129.59,129.85,129.94,130.16$, $130.32,130.42$, 130.70, 130.78, 131.03, 131.45, 131.56, 132.09, 132.31, 135.02, 136.91, 138.83, 139.15, 139.69, 139.79, 158.61, 158.76, 159.03, 164.87, 172.74, 172.96. HRMS (FAB) calcd for $\mathrm{C}_{39} \mathrm{H}_{36} \mathrm{~N}_{2} \mathrm{O}_{3} \mathrm{Cl}[\mathrm{M}+\mathrm{H}]^{+}:$615.2414. Found: $615.2424(+1.5 \mathrm{ppm},+0.9 \mathrm{mmu})$. HPLC $\mathrm{t}_{\mathrm{r}} 27.2 \mathrm{~min}$ $(S)$; $\mathrm{t}_{\mathrm{r}} 34.6 \mathrm{~min}(R)$ [Chiralpak AD $(0.46 \mathrm{~cm} \times 25 \mathrm{~cm})$ (from Daicel Chemical Ind., Ltd.) Hexane $/ i$-PrOH, 97/3, $1.0 \mathrm{~mL} / \mathrm{min}, 94 \%$ ee.

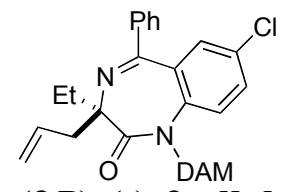

(3R)-(-)-3-allyl-1-di(p-anisyl)methyl-7-chloro-3-ethyl-1,3-dihydro-5-phenyl-2H-1,4benzodiazepin-2-one [(R)-(-)-21d]

At $-42^{\circ} \mathrm{C}$ under nitrogen, to a stirred solution of $(S)-7 \mathbf{d}(50.0 \mathrm{mg}, 0.095 \mathrm{mmol}, 1.0$ equiv) and HMPA (100 $\mu \mathrm{L}, 0.572 \mathrm{mmol}, 6.0$ equiv) in anhydrous DME $(2.8 \mathrm{~mL})$ was added KHMDS 
( $0.764 \mathrm{~mL}, 0.382 \mathrm{mmol}, 4.0$ equiv, $0.5 \mathrm{M}$ in toluene). The resulting solution was stirred for a further $30.0 \mathrm{~min}$ at $-42^{\circ} \mathrm{C}$ and then transferred via a cannula into a solution of allyl iodide (86.9 $\mu \mathrm{L}, 0.950 \mathrm{mmol}, 10.0$ equiv) and HMPA $(50 \mu \mathrm{L}, 0.286 \mathrm{mmol})$ in dried DME $(1.4 \mathrm{~mL})$ at $-42^{\circ} \mathrm{C}$. The reaction was stirred at $-42^{\circ} \mathrm{C}$ for $2.2 \mathrm{~h}$ at which the starting benzodiazepine $(S)-7 \mathbf{d}$ was consumed (TLC, 1:3 EtOAc/hexanes). The reaction was quenched at $-42^{\circ} \mathrm{C}$ with sat. $\mathrm{NH}_{4} \mathrm{Cl}$ (aq) $(5.0 \mathrm{~mL})$ and extracted with $\mathrm{CH}_{2} \mathrm{Cl}_{2}(3 \times 5 \mathrm{~mL})$. The combined organic extracts were dried over anhydrous $\mathrm{Na}_{2} \mathrm{SO}_{4}$, filtered, and concentrated under reduced pressure. The crude product was purified by flash chromatography on silica gel (1:3 EtOAc/hexanes) to afford $31.1 \mathrm{mg}(58 \%)$ of $(R)-(-)-21 d$ as clear yellow oil. $[\alpha]_{\mathrm{D}}{ }^{25}=-14.7\left(c 1.56, \mathrm{CHCl}_{3}\right) .{ }^{1} \mathrm{H} \mathrm{NMR}\left(\mathrm{CDCl}_{3}\right)$ indicated a 60:40 mixture of axial-Et and equatorial-Et conformers $\delta 0.77-.82(\mathrm{~m}, 3 \mathrm{H} \times 0.60 \mathrm{ax}-\mathrm{Et}), 1.18$ $1.22(\mathrm{~m}, 3 \mathrm{H} \times 0.40 \mathrm{eq}-\mathrm{Et}), 1.25-1.32(\mathrm{~m}, 1 \mathrm{H} \times 0.60 \mathrm{ax}-\mathrm{Et}), 1.32-1.40(\mathrm{~m}, 1 \mathrm{H} \times 0.60 \mathrm{ax}-\mathrm{Et}$, overlapping with peaks at 1.32-1.40), $2.00\left(\mathrm{dd},{ }^{2} J_{\mathrm{HH}}=13.8 \mathrm{~Hz},{ }^{3} J_{\mathrm{HH}}=8.0 \mathrm{~Hz}, 1 \mathrm{H} \times 0.40 \mathrm{eq}-\mathrm{Et}\right)$, $2.12\left(\mathrm{dd},{ }^{2} J_{\mathrm{HH}}=14.8, \mathrm{~Hz}^{3} J_{\mathrm{HH}}=6.4 \mathrm{~Hz}, 1 \mathrm{H} \times 0.40 \mathrm{eq}-\mathrm{Et}\right), 2.21-2.27(\mathrm{~m}, 1 \mathrm{H} \times 0.40 \mathrm{eq}-\mathrm{Et}), 2.37$ $\left(\mathrm{dd},{ }^{2} J_{\mathrm{HH}}=9.2 \mathrm{~Hz},{ }^{3} J_{\mathrm{HH}}=2.0 \mathrm{~Hz}, 1 \mathrm{H} \times 0.40 \mathrm{eq}-\mathrm{Et}\right), 2.99\left(\mathrm{dd},{ }^{2} J_{\mathrm{HH}}=8.6 \mathrm{~Hz},{ }^{3} J_{\mathrm{HH}}=2.4 \mathrm{~Hz}, 1 \mathrm{H} \times\right.$ $0.60 \mathrm{ax}-\mathrm{Et}), 3.10\left(\mathrm{~d},{ }^{2} J_{\mathrm{HH}}=13.2 \mathrm{~Hz}, 1 \mathrm{H} \times 0.60 \mathrm{ax}-\mathrm{Et}\right), 3.76(\mathrm{~d}, 2$ conformers overlapping, $3 \mathrm{H})$, $3.84(\mathrm{~m}, 2$ conformers overlapping, $3 \mathrm{H}), 4.76\left(\mathrm{~d},{ }^{2} J_{\mathrm{HH}}=16.0 \mathrm{~Hz}, 1 \mathrm{H} \times 0.40 \mathrm{eq}-\mathrm{Et}\right), 5.08\left(\mathrm{~d},{ }^{2} J_{\mathrm{HH}}\right.$ $=9.2 \mathrm{~Hz}, 1 \mathrm{H} \times 0.40 \mathrm{eq}-\mathrm{Et}), 5.19\left(\mathrm{~d},{ }^{2} J_{\mathrm{HH}}=10.0 \mathrm{~Hz}, 1 \mathrm{H} \times 0.60 \mathrm{ax}-\mathrm{Et}\right), 5.26\left(\mathrm{~d},{ }^{2} J_{\mathrm{HH}}=17.2 \mathrm{~Hz}\right.$, $1 \mathrm{H} \times 0.60 \mathrm{ax}-\mathrm{Et}), 5.68-5.76(\mathrm{~m}, 1 \mathrm{H} \times 0.40 \mathrm{eq}-\mathrm{Et}), 6.23-6.33(\mathrm{~m}, 1 \mathrm{H} \times 0.60 \mathrm{ax}-\mathrm{Et}), 6.66\left(\mathrm{dd},{ }^{3} J_{\mathrm{HH}}\right.$ $\left.=13.3 \mathrm{~Hz},{ }^{4} J_{\mathrm{HH}}=2.4 \mathrm{~Hz}, 2 \mathrm{H}\right), 6.89-6.97(\mathrm{~m}, 4 \mathrm{H}), 6.99-7.02(\mathrm{~m}, 2 \mathrm{H}), 7.08-7.13(\mathrm{~m}, 1 \mathrm{H}), 7.15-$ $7.18(\mathrm{~m}, 1 \mathrm{H}), 7.24-7.27(\mathrm{~m}, 2 \mathrm{H}), 7.31-7.39(\mathrm{~m}, 4 \mathrm{H}), 7.45\left(\mathrm{t},{ }^{3} J_{\mathrm{HH}}=6.4 \mathrm{~Hz}, 1 \mathrm{H}\right) .{ }^{13} \mathrm{C} \mathrm{NMR}$ $\left(\mathrm{CDCl}_{3}\right)$ was consistent with a 60:40 mixture of axial-Et and equatorial-Et conformers (48 resonances found for a possible $2 \times 29$ unique carbons): $\delta 8.12,9.22,22.03,31.26,33.15,42.80$, $55.31,55.42$, 65.95, 66.01, 69.62, 70.76, 113.59, 113.62, 113.79, 113.92, 117.63, 118.30, 125.77, $125.96,128.28,128.72,128.92,129.06,129.45,129.73,129.94,130.00,130.40,130.70,130.77$, $131.47,133.28,134.51,134.57,135.97,139.29,139.38,139.57,139.70,158.69,158.72,158.94$, 158.97, 165.17, 165.46, 172.69, 172.74. HRMS (FAB) calcd for $\mathrm{C}_{35} \mathrm{H}_{34} \mathrm{~N}_{2} \mathrm{O}_{3} \mathrm{Cl}[\mathrm{M}+\mathrm{H}]^{+}$: 565.2258. Found: $565.2281(+4.1 \mathrm{ppm},+2.3 \mathrm{mmu})$. HPLC $\mathrm{t}_{\mathrm{r}} 21.2 \mathrm{~min}(S) ; \mathrm{t}_{\mathrm{r}} 23.7 \mathrm{~min}(R)$ [Chiralcel OD $(0.46 \mathrm{~cm} \times 25 \mathrm{~cm})$ (from Daicel Chemical Ind., Ltd.) Hexane/ $i-\mathrm{PrOH}, 99 / 1,1.0$ $\mathrm{mL} / \mathrm{min}, 94 \%$ ee.<smiles>CCN1C(=O)N(OC)c2ccc(Cl)cc2C(c2ccccc2)N=C1O</smiles>

(3R)-(+)-1-di(p-anisyl)methyl-7-chloro-3-cyano-3-ethyl-1,3-dihydro-5-phenyl-2H-1,4benzodiazepin-2-one $[(R)-(+)-22 \mathrm{~d}]$

At $-42{ }^{\circ} \mathrm{C}$ under nitrogen, to a stirred solution of $(S)-7 \mathbf{d}(50.0 \mathrm{mg}, 0.095 \mathrm{mmol}, 1.0$ equiv) and HMPA (100 $\mu \mathrm{L}, 0.572 \mathrm{mmol}, 6.0$ equiv) in anhydrous DME $(2.8 \mathrm{~mL})$ was added KHMDS ( $0.764 \mathrm{~mL}, 0.382 \mathrm{mmol}, 4.0$ equiv, $0.5 \mathrm{M}$ in toluene). The resulting solution was stirred for a further $30.0 \mathrm{~min}$ at $-42^{\circ} \mathrm{C}$ and then transferred via a cannula into a solution of $p$-toluenesulfonyl cyanide (37.1 mg, $0.950 \mathrm{mmol}, 2.0$ equiv) and HMPA (50 $\mu \mathrm{L}, 0.286 \mathrm{mmol})$ in dried DME (1.4 $\mathrm{mL}$ ) at $-42^{\circ} \mathrm{C}$. The reaction was stirred at $-42^{\circ} \mathrm{C}$ for $33 \mathrm{~min}$ at which the starting benzodiazepine $(S)$-7d was consumed (TLC, 1:20 Et ${ }_{2} \mathrm{O} / \mathrm{DCM}$ ). The reaction was quenched at $42{ }^{\circ} \mathrm{C}$ with sat. $\mathrm{NH}_{4} \mathrm{Cl}(\mathrm{aq})(5.0 \mathrm{~mL})$ and extracted with $\mathrm{CH}_{2} \mathrm{Cl}_{2}(3 \times 5 \mathrm{~mL})$. The combined organic extracts were dried over anhydrous $\mathrm{Na}_{2} \mathrm{SO}_{4}$, filtered, and concentrated under reduced pressure. The crude product was purified by flash chromatography on silica gel ( $1^{\text {st }}$ eluent 1:20 
$\mathrm{Et}_{2} \mathrm{O} / \mathrm{DCM}, 2^{\text {nd }}$ eluent 1:3 EtOAc/hexanes) to afford $45.0 \mathrm{mg}(86 \%)$ of $(R)-(+)-22 \mathrm{~d}$ as clear yellow oil. $[\alpha]_{\mathrm{D}}{ }^{25}=+143\left(c=2.25, \mathrm{CHCl}_{3}\right) .{ }^{1} \mathrm{H} \mathrm{NMR}\left(\mathrm{CDCl}_{3}\right) \delta 1.34\left(\mathrm{t},{ }^{3} J_{\mathrm{HH}}=7.5 \mathrm{~Hz}, 3 \mathrm{H}\right)$, 2.48-2.54 (m, 1H), 2.63-2.69 (m, 1H), $3.76(\mathrm{~d}, 2$ conformers, 3H), $3.84(\mathrm{~d}, 2$ conformers, $3 \mathrm{H})$, $6.66\left(\mathrm{dd},{ }^{3} J_{\mathrm{HH}}=8.0 \mathrm{~Hz},{ }^{4} J_{\mathrm{HH}}=2.8 \mathrm{~Hz}, 2 \mathrm{H}\right), 6.90-6.97(\mathrm{~m}, 4 \mathrm{H}), 7.01(\mathrm{~s}, 1 \mathrm{H}), 7.12(\mathrm{~s}, 1 \mathrm{H}), 7.24-$ $7.30(\mathrm{~m}, 4 \mathrm{H}), 7.33-7.35(\mathrm{~m}, 2 \mathrm{H}), 7.38-7.43(\mathrm{~m}, 2 \mathrm{H}), 7.50-7.53(\mathrm{~m}, 1 \mathrm{H}) .{ }^{13} \mathrm{C} \mathrm{NMR}\left(\mathrm{CDCl}_{3}\right)$ One conformer $\mathrm{CN}$ axial ( 26 resonances found for a possible 27 unique carbons): $\delta 8.39,31.86$, 55.33, 55.46, 63.28, 65.75, 113.81, 114.20, 115.02, 126.28, 128.55, 128.81, 129.40, 129.61, 129.89, 131.38, 131.55, 131.71, 131.76, 132.73, 137.43, 138.85, 159.06, 159.29, 164.90, 170.47. HRMS (FAB) calcd for $\mathrm{C}_{33} \mathrm{H}_{29} \mathrm{~N}_{3} \mathrm{O}_{3} \mathrm{Cl}[\mathrm{M}+\mathrm{H}]^{+}: 550.1897$. Found: $550.1876(-3.9 \mathrm{ppm},-2.1$ $\mathrm{mmu}$ ). HPLC $\mathrm{t}_{\mathrm{r}} 21.5 \mathrm{~min}(R) ; \mathrm{t}_{\mathrm{r}} 26.5 \mathrm{~min}(S)$ [Chiralcel OD $(0.46 \mathrm{~cm} \times 25 \mathrm{~cm})$ (from Daicel Chemical Ind., Ltd.) Hexane $/ i$-PrOH, 99/1, $1.0 \mathrm{~mL} / \mathrm{min}, 96 \%$ ee.

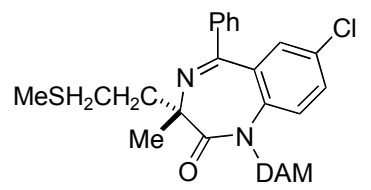

(3S)-(-)-1-di(p-anisyl)methyl-7-chloro-1,3-dihydro-3-methyl-5-phenyl-3-(2thiomethyl)ethyl-2H-1,4-benzodiazepin-2-one [(S)-(-)-23d]

At $-42{ }^{\circ} \mathrm{C}$ under nitrogen, to a stirred solution of $(S)-8 d(50.0 \mathrm{mg}, 0.088 \mathrm{mmol}, 1.0$ equiv) and HMPA ( $92 \mu \mathrm{L}, 0.528 \mathrm{mmol}, 6.0$ equiv) in anhydrous DME (2.6 mL) was added KHMDS (0.704 $\mathrm{mL}, 0.352 \mathrm{mmol}, 4.0$ equiv, $0.5 \mathrm{M}$ in toluene). The resulting solution was stirred for a further $30.0 \mathrm{~min}$ at $-42^{\circ} \mathrm{C}$ and then transferred via a cannula into a solution of methyl iodide $(55 \mu \mathrm{L}$, $0.880 \mathrm{mmol}, 10.0$ equiv) and HMPA $(46 \mu \mathrm{L}, 0.264 \mathrm{mmol})$ in dried DME $(1.3 \mathrm{~mL})$ at $-42{ }^{\circ} \mathrm{C}$. The reaction was stirred at $-42^{\circ} \mathrm{C}$ for $1.3 \mathrm{~h}$ at which the starting benzodiazepine $(S)$-8d was consumed (TLC, 1:3 EtOAc/hexanes). The reaction was quenched at $-42^{\circ} \mathrm{C}$ with sat. $\mathrm{NH}_{4} \mathrm{Cl}$ (aq) $(5.0 \mathrm{~mL})$ and extracted with $\mathrm{CH}_{2} \mathrm{Cl}_{2}(3 \times 5 \mathrm{~mL})$. The combined organic extracts were dried over anhydrous $\mathrm{Na}_{2} \mathrm{SO}_{4}$, filtered, and concentrated under reduced pressure. The crude product was purified by flash chromatography on silica gel (1:3 EtOAc/hexanes) to afford $38.3 \mathrm{mg}$ $(67 \%)$ of $(S)-(-)-23 d$ as clear yellow oil. $[\alpha]_{\mathrm{D}}{ }^{25}=-72.6\left(c=1.92, \mathrm{CHCl}_{3}\right) .{ }^{1} \mathrm{H} \mathrm{NMR}\left(\mathrm{CDCl}_{3}\right)$ indicated a 65:35 mixture of axial-Me and equatorial-Me conformers $\delta 1.04(\mathrm{~s}, 3 \mathrm{H} \times 0.65 \mathrm{ax}-$ $\mathrm{Me}), 1.59-1.76(\mathrm{~m}, 2 \mathrm{H} \times 0.35$ eq-Me $), 1.78(\mathrm{~s}, 3 \mathrm{H} \times 0.35 \mathrm{eq}-\mathrm{Me}), 1.91(\mathrm{~s}, 3 \mathrm{H} \times 0.35$ eq-Me), $2.20(\mathrm{~s}, 3 \mathrm{H} \times 0.65 \mathrm{ax}-\mathrm{Me}), 2.23-2.48(\mathrm{~m}, 2 \mathrm{H} \times 0.65 \mathrm{ax}-\mathrm{Me}), 2.65-2.73(\mathrm{~m}, 2 \mathrm{H} \times 0.35 \mathrm{eq}-\mathrm{Me})$, $2.84-3.00(\mathrm{~m}, 2 \mathrm{H} \times 0.65 \mathrm{ax}-\mathrm{Me}), 3.76(\mathrm{~d}, 2$ conformers, $3 \mathrm{H}), 3.84(\mathrm{~s}, 3 \mathrm{H}), 6.67\left(\mathrm{dd},{ }^{3} J_{\mathrm{HH}}=8.6\right.$ $\left.\mathrm{Hz},{ }^{4} J_{\mathrm{HH}}=3.6 \mathrm{~Hz}, 2 \mathrm{H}\right), 6.92-6.97(\mathrm{~m}, 4 \mathrm{H}), 6.99-7.03(\mathrm{~m}, 2 \mathrm{H}), 7.10-7.24(\mathrm{~m}, 4 \mathrm{H}), 7.32-7.40(\mathrm{~m}$, $4 \mathrm{H}), 7.44-7.46(\mathrm{~m}, 1 \mathrm{H}) .{ }^{13} \mathrm{C} \mathrm{NMR}\left(\mathrm{CDCl}_{3}\right)$ was consistent with a $65: 35$ mixture of axial-Me and equatorial-Me conformers (47 resonances found for a possible $2 \times 28$ unique carbons): $\delta 15.37$, $15.63,18.63,28.36,29.23,29.40,32.74,43.32,55.31,55.43,65.40,65.87,65.98,67.55,113.61$, $113.67,113.99,125.64,125.85,128.29,128.38,128.84,128.88,128.92,128.98,129.45,129.57$, 129.91, 130.06, 130.26, 130.51 (2 overlapping peaks), 130.58, 130.60, 131.38, 131.43, 132.32, 134.13, 139.24, 139.37, 139.46, 158.74, 158.94, 159.02, 165.50, 165.96, 172.50, 172.99. HRMS (FAB) calcd for $\mathrm{C}_{34} \mathrm{H}_{34} \mathrm{~N}_{2} \mathrm{O}_{3} \mathrm{ClS}[\mathrm{M}+\mathrm{H}]^{+}: 585.1979$. Found: $585.2003(+4.2 \mathrm{ppm},+2.4 \mathrm{mmu})$. HPLC $\mathrm{t}_{\mathrm{r}} 20.5 \mathrm{~min}(R) ; \mathrm{t}_{\mathrm{r}} 25.1 \mathrm{~min}(S)$ [Chiralpak AD $(0.46 \mathrm{~cm} \times 25 \mathrm{~cm})$ (from Daicel Chemical Ind., Ltd.) Hexane/i-PrOH, 97/3, $1.0 \mathrm{~mL} / \mathrm{min}, 87 \%$ ee. 


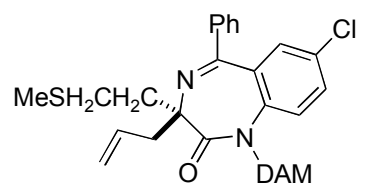

(3S)-(-)-3-allyl-1-di(p-anisyl)methyl-7-chloro-1,3-dihydro-5-phenyl-3-(2-thiomethyl)ethyl2H-1,4-benzodiazepin-2-one [(S)-(-)-24d]

At $-42{ }^{\circ} \mathrm{C}$ under nitrogen, to a stirred solution of $(S)-8 d(50.0 \mathrm{mg}, 0.088 \mathrm{mmol}, 1.0$ equiv) and HMPA (92 $\mu \mathrm{L}, 0.528 \mathrm{mmol}, 6.0$ equiv) in anhydrous DME (2.6 mL) was added KHMDS (0.704 $\mathrm{mL}, 0.352 \mathrm{mmol}, 4.0$ equiv, $0.5 \mathrm{M}$ in toluene). The resulting solution was stirred for a further 30.0 min at $-42^{\circ} \mathrm{C}$ and then transferred via a cannula into a solution of allyl bromide $(77 \mu \mathrm{L}$, $0.880 \mathrm{mmol}, 10.0$ equiv) and HMPA $(46 \mu \mathrm{L}, 0.264 \mathrm{mmol})$ in dried DME $(1.3 \mathrm{~mL})$ at $-42^{\circ} \mathrm{C}$. The reaction was stirred at $-42^{\circ} \mathrm{C}$ for $3.4 \mathrm{~h}$ (TLC, $1: 3 \mathrm{EtOAc} /$ hexanes). The reaction was quenched at $-42{ }^{\circ} \mathrm{C}$ with sat. $\mathrm{NH}_{4} \mathrm{Cl}$ (aq) $(5.0 \mathrm{~mL})$ and extracted with $\mathrm{CH}_{2} \mathrm{Cl}_{2}(3 \times 5 \mathrm{~mL})$. The combined organic extracts were dried over anhydrous $\mathrm{Na}_{2} \mathrm{SO}_{4}$, filtered, and concentrated under reduced pressure. The crude product was purified by flash chromatography on silica gel (1:3 EtOAc/hexanes) to afford $17.6 \mathrm{mg}(33 \%)$ of $(S)-(-)-24 d$ as clear yellow oil. $[\alpha]_{\mathrm{D}}{ }^{25}=-71.4(c=$ $\left.0.88, \mathrm{CHCl}_{3}\right) .{ }^{1} \mathrm{H} \mathrm{NMR}\left(\mathrm{CDCl}_{3}\right)$ indicated a 60:40 mixture of axial-allyl and equatorial-allyl conformers $\delta 1.52-1.73(\mathrm{~m}, 2 \mathrm{H} \times 0.40$ eq-allyl), $1.81(\mathrm{~s}, 3 \mathrm{H} \times 0.40$ eq-allyl $), 2.09-2.11(\mathrm{~m}, 2 \mathrm{H} \times$ 0.60 ax-allyl), 2.20 (s, $3 \mathrm{H} \times 0.60$ ax-allyl), 2.33-2.41 (m, $2 \mathrm{H} \times 0.60$ ax-allyl), $2.67-2.74(\mathrm{~m}, 2 \mathrm{H} \times$ 0.40 eq-allyl), 2.88-2.96 (m, $2 \mathrm{H} \times 0.60 \mathrm{ax}$-allyl), $3.05\left(\mathrm{dd},{ }^{2} J_{\mathrm{HH}}=14.4 \mathrm{~Hz},{ }^{3} J_{\mathrm{HH}}=8.0 \mathrm{~Hz}, 1 \mathrm{H} \times\right.$ 0.40 eq-allyl), $3.11\left(\mathrm{dd},{ }^{2} J_{\mathrm{HH}}=14.6 \mathrm{~Hz},{ }^{3} J_{\mathrm{HH}}=5.6 \mathrm{~Hz}, 1 \mathrm{H} \times 0.40\right.$ eq-allyl), $3.76(\mathrm{~s}, 3 \mathrm{H}), 3.84-$ $3.85(\mathrm{~d}, 2$ overlapping conformers, $3 \mathrm{H}), 4.76\left(\mathrm{~d},{ }^{2} J_{\mathrm{HH}}=17.0 \mathrm{~Hz}, 1 \mathrm{H} \times 0.60\right.$ ax-allyl $), 5.09(\mathrm{~d}$, ${ }^{2} J_{\mathrm{HH}}=9.8 \mathrm{~Hz}, 1 \mathrm{H} \times 0.60 \mathrm{ax}$-allyl $), 5.21\left(\mathrm{~d},{ }^{2} J_{\mathrm{HH}}=10.4 \mathrm{~Hz}, 1 \mathrm{H} \times 0.40\right.$ eq-allyl $), 5.26\left(\mathrm{~d},{ }^{2} J_{\mathrm{HH}}=\right.$ $10.0 \mathrm{~Hz}, 1 \mathrm{H} \times 0.40$ eq-allyl), 5.65-5.76 (m, $1 \mathrm{H} \times 0.60$ ax-allyl), $6.23-6.33(\mathrm{~m}, 1 \mathrm{H} \times 0.40 \mathrm{eq}-$ allyl), $6.66\left(\mathrm{~d},{ }^{3} J_{\mathrm{HH}}=8.8 \mathrm{~Hz}, 2 \mathrm{H}\right), 6.90-6.96(\mathrm{~m}, 5 \mathrm{H}), 7.02\left(\mathrm{~d},{ }^{4} J_{\mathrm{HH}}-2.4 \mathrm{~Hz}, 1 \mathrm{H}\right), 7.10-7.18(\mathrm{~m}$, $2 \mathrm{H}), 7.23-7.26\left(\mathrm{~d},{ }^{3} J_{\mathrm{HH}}=10.8 \mathrm{~Hz}, 2 \mathrm{H}\right), 7.30-7.39(\mathrm{~m}, 4 \mathrm{H}), 7.46\left(\mathrm{t},{ }^{3} J_{\mathrm{HH}}=6.8 \mathrm{~Hz}, 1 \mathrm{H}\right) .{ }^{13} \mathrm{C} \mathrm{NMR}$ $\left(\mathrm{CDCl}_{3}\right)$ was consistent with a 60:40 mixture of axial-allyl and equatorial-allyl conformers (48 resonances found for a possible $2 \times 30$ unique carbons): $\delta 15.42,15.64,28.99,29.35,29.40$, $35.31,39.85,43.74,55.31,55.43,66.08,69.55,69.58,113.61,113.67,113.85,113.99,118.15$, $118.86,125.97,128.27,128.35,128.88,128.92,129.00,129.45,129.86,130.05,130.23,130.40$, 130.52 , 130.61, 131.44, 132.64, 134.28, 135.55, 139.16, 139.20, 139.28, 139.34, 158.74, 158.79, 158.98, 159.03, 165.50, 165.65, 171.99, 172.26. HRMS (FAB) calcd for $\mathrm{C}_{36} \mathrm{H}_{36} \mathrm{~N}_{2} \mathrm{O}_{3} \mathrm{ClS}$ $[\mathrm{M}+\mathrm{H}]^{+}: 611.2135$. Found: $611.2168(+5.4 \mathrm{ppm},+3.3 \mathrm{mmu})$. HPLC $\mathrm{t}_{\mathrm{r}} 16.7 \mathrm{~min}(S) ; \mathrm{t}_{\mathrm{r}} 20.7 \mathrm{~min}$ $(R)$ [Chiralpak AD-H $(0.46 \mathrm{~cm} \times 25 \mathrm{~cm})$ (from Daicel Chemical Ind., Ltd.) Hexane $/ i$-PrOH, $97 / 3,1.0 \mathrm{~mL} / \mathrm{min}, 72 \%$ ee.

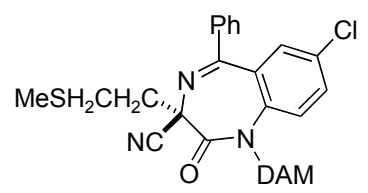

(3R)-(-)-1-di(p-anisyl)methyl-7-chloro-3-cyano-1,3-dihydro-5-phenyl-3-(2-thiomethyl)ethyl2H-1,4-benzodiazepin-2-one [(R)-(-)-25d]

At $-42{ }^{\circ} \mathrm{C}$ under nitrogen, to a stirred solution of $(S)-8 d(50.0 \mathrm{mg}, 0.088 \mathrm{mmol}, 1.0$ equiv) and HMPA (92 $\mu \mathrm{L}, 0.528 \mathrm{mmol}, 6.0$ equiv) in anhydrous DME (2.6 mL) was added KHMDS (0.704 $\mathrm{mL}, 0.352 \mathrm{mmol}, 4.0$ equiv, $0.5 \mathrm{M}$ in toluene). The resulting solution was stirred for a further $30.0 \mathrm{~min}$ at $-42^{\circ} \mathrm{C}$ and then transferred via a cannula into a solution of $p$-toluenesulfonyl cyanide (31.9 mg, $0.176 \mathrm{mmol}, 2.0$ equiv) and HMPA (46 $\mu \mathrm{L}, 0.264 \mathrm{mmol})$ in dried DME (1.3 mL) at - 
$42^{\circ} \mathrm{C}$. The reaction was stirred at $-42^{\circ} \mathrm{C}$ for $30 \mathrm{~min}$ at which the starting benzodiazepine $(S)-(-)-$ 8d was consumed (TLC, 1:3 EtOAc/hexanes). The reaction was quenched at $-42{ }^{\circ} \mathrm{C}$ with sat. $\mathrm{NH}_{4} \mathrm{Cl}(\mathrm{aq})(5.0 \mathrm{~mL})$ and extracted with $\mathrm{CH}_{2} \mathrm{Cl}_{2}(3 \times 5 \mathrm{~mL})$. The combined organic extracts were dried over anhydrous $\mathrm{Na}_{2} \mathrm{SO}_{4}$, filtered, and concentrated under reduced pressure. The crude product was purified by flash chromatography on silica gel (1:3 EtOAc/hexanes) to afford $41.7 \mathrm{mg}(80 \%)$ of $(S)-(-)-25 d$ as clear yellow oil. $[\alpha]_{\mathrm{D}}{ }^{25}=-121\left(c 2.09, \mathrm{CHCl}_{3}\right) .{ }^{1} \mathrm{H} \mathrm{NMR}$ $\left(\mathrm{CDCl}_{3}\right) \delta 2.24(\mathrm{~s}, 3 \mathrm{H}), 2.75-3.04(\mathrm{~m}, 4 \mathrm{H}), 3.77(\mathrm{~s}, 3 \mathrm{H}), 3.85(\mathrm{~s}, 3 \mathrm{H}), 6.66\left(\mathrm{~d},{ }^{2} J_{\mathrm{HH}}=8.8 \mathrm{~Hz}\right.$, $2 \mathrm{H}), 6.89-6.99(\mathrm{~m}, 5 \mathrm{H}), 7.12(\mathrm{~s}, 1 \mathrm{H}), 7.24-7.28(\mathrm{~m}, 4 \mathrm{H}), 7.32-7.34(\mathrm{~m}, 2 \mathrm{H}), 7.41\left(\mathrm{t},{ }^{3} J_{\mathrm{HH}}=8.0\right.$ $\mathrm{Hz}, 2 \mathrm{H}), 7.51-7.55(\mathrm{~m}, 1 \mathrm{H}) .{ }^{13} \mathrm{C} \mathrm{NMR}\left(\mathrm{CDCl}_{3}\right)$ One conformer $\mathrm{CN}$ axial $(26$ resonances found for a possible 28 unique carbons): $\delta 15.67,28.54,38.52,55.34,55.47,62.04,65.91,113.84$, $114.23,114.73,126.33,128.58,128.79,129.25,129.43,129.94,131.39,131.74,131.90,132.61$, 137.15, 138.66, 159.11, 159.34, 163.69, 170.63. HRMS (FAB) calcd for $\mathrm{C}_{34} \mathrm{H}_{31} \mathrm{~N}_{3} \mathrm{O}_{3} \mathrm{ClS}$ $[\mathrm{M}+\mathrm{H}]^{+}: 596.1775$. Found: $596.1823(+8.1 \mathrm{ppm},+4.8 \mathrm{mmu})$. HPLC $\mathrm{t}_{\mathrm{r}} 9.0 \mathrm{~min}(R) ; \mathrm{t}_{\mathrm{r}} 14.3 \mathrm{~min}$ $(S)$ [Chiralcel OD $(0.46 \mathrm{~cm} \times 25 \mathrm{~cm})$ (from Daicel Chemical Ind., Ltd.) Hexane $/ i$-PrOH, 90/10, $1.0 \mathrm{~mL} / \mathrm{min}, 87 \%$ ee.

\section{E. Removal of the DAM group from quaternary 1,4-benzodiazepin-2-ones General procedure}

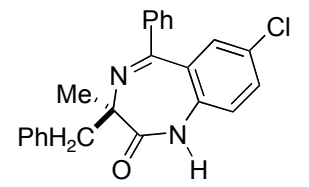

$(R)-(+)-3-b e n z y l-7-c h l o r o-1,3-d i h y d r o-3-m e t h y l-5-p h e n y l-2 H-1,4-b e n z o d i a z e p i n-2-o n e ~[(R)-$ $(+)-2 \mathrm{c}]$

Compound (R)-(-)-2d (60 mg, $0.1 \mathrm{mmol})$ was dissolved in a solution of 25\% TFA in $\mathrm{CH}_{2} \mathrm{Cl}_{2}(3.4$ $\mathrm{mL}$ ) and stirred for $1 \mathrm{~h}$. The excess solvent was removed in vacuo and partitioned between anhydrous ether and 2:1 saturated $\mathrm{K}_{2} \mathrm{CO}_{3} /$ water for $30 \mathrm{~min}$. The aqueous layer was then extracted with ether $(10 \mathrm{~mL} \times 3)$, and the combined organic extracts were dried over anhydrous $\mathrm{Na}_{2} \mathrm{SO}_{4}$, filtered, and concentrated in vacuo. The crude product was purified with flash column chromatography on $\mathrm{C} 18\left(80: 20 \mathrm{CH}_{3} \mathrm{OH} / \mathrm{H}_{2} \mathrm{O}\right)$ to afford $36 \mathrm{mg}(96 \%)$ of $(R)-(+)-\mathbf{2 c}$ as colorless semi-solid. $[\alpha]_{\mathrm{D}}{ }^{23}=+29.3\left(\mathrm{c}=2.5, \mathrm{CHCl}_{3}\right) .{ }^{1} \mathrm{H} \mathrm{NMR}\left(\mathrm{CDCl}_{3}\right): \delta 9.51(\mathrm{~s}, \mathrm{NH}), 7.51-7.37(\mathrm{~m}, 6 \mathrm{H})$, $7.32(\mathrm{~m}, 5 \mathrm{H}), 7.03(\mathrm{~d}, J=8.7 \mathrm{~Hz}, 2 \mathrm{H}), 3.17(\mathrm{~d}, J=13.3 \mathrm{~Hz}, 1 \mathrm{H}), 3.03(\mathrm{~d}, J=13.5 \mathrm{~Hz}, 1 \mathrm{H}), 1.39$ (s, 3H). ${ }^{13} \mathrm{C} \mathrm{NMR}\left(\mathrm{CDCl}_{3}\right): \delta 174.7,166.0,140.7,137.0,132.0,131.1,130.5,130.3,129.9,129.3$, 128.4, 128.1, 127.9, 126.7, 121.6, 66.7, 41.9 (broad peak), 22.4 (broad peak). HRMS calcd. for $\mathrm{C}_{23} \mathrm{H}_{20} \mathrm{ClN}_{2} \mathrm{O}(\mathrm{M}+1)$ 375.1264, found $375.1281(+4.5 \mathrm{ppm},+1.7 \mathrm{mmu})$. HPLC $\mathrm{t}_{\mathrm{r}} 11.3 \mathrm{~min}(S) ; \mathrm{t}_{\mathrm{r}}$ $14.5(R) \min$ [Chiralpak AD $(0.46 \mathrm{~cm} \times 25 \mathrm{~cm})$ (from Daicel Chemical Ind., Ltd.)] Hexane/i$\mathrm{PrOH}, 95 / 5,1.0 \mathrm{~mL} / \mathrm{min}, 100 \%$ ee.

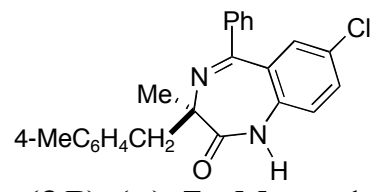

(3R)-(+)-7-chloro-1,3-dihydro-3-methyl-3-(4-methylbenzyl)-5-phenyl-2H-1,4benzodiazepin-2-one $[(R)-9 \mathrm{c}]$ 
The general procedure of was followed with $(R)-(-)-9 d(64.0 \mathrm{mg}, 0.104 \mathrm{mmol})$ and 25\% TFA in $\mathrm{CH}_{2} \mathrm{Cl}_{2}(3.4 \mathrm{~mL})$. The reaction mixture was stirred at r.t. for $1 \mathrm{~h}$. Purification with flash column chromatography on $\mathrm{C} 18\left(80: 20 \mathrm{CH}_{3} \mathrm{OH} / \mathrm{H}_{2} \mathrm{O}\right)$ to afford $39 \mathrm{mg}(96 \%)$ of $(R)-(+)-9 \mathrm{c}$ as colorless semi-solid. $[\alpha]_{\mathrm{D}}{ }^{23}=+47.1\left(\mathrm{c}=1.4, \mathrm{CHCl}_{3}\right){ }^{1} \mathrm{H} \mathrm{NMR}\left(\mathrm{CDCl}_{3}\right): \delta 9.42(\mathrm{~s}, \mathrm{NH}), 7.51-7.43(\mathrm{~m}, 3 \mathrm{H})$, 7.43-7.37 (m, 3H), $7.24(\mathrm{~d}, \mathrm{~J}=2.3 \mathrm{~Hz}, 1 \mathrm{H}), 7.12(\mathrm{AB}$ pattern, $J=7.8 \mathrm{~Hz}, 4 \mathrm{H}), 7.03(\mathrm{~d}, J=8.5 \mathrm{~Hz}$, $1 \mathrm{H}), 3.08(\mathrm{~d}, J=12.8 \mathrm{~Hz}, 1 \mathrm{H}), 2.98(\mathrm{~d}, J=13.5 \mathrm{~Hz}, 1 \mathrm{H}), 2.32(\mathrm{~s}, 3 \mathrm{H}) .{ }^{13} \mathrm{C} \mathrm{NMR}\left(\mathrm{CDCl}_{3}\right): \delta 174.7$, $165.9,140.8,137.0,136.2,133.9,131.9,130.9,130.5,130.3,129.8,129.3,128.7,128.3,128.1$, $121.5,66.8,41.0$ (broad peak), 22.6 (broad peak), 21.1. HRMS calcd. for $\mathrm{C}_{24} \mathrm{H}_{22} \mathrm{ClN}_{2} \mathrm{O}(\mathrm{M}+1)$ 389.1421, found 389.1416 (-1.2 ppm, $-0.5 \mathrm{mmu})$. HPLC $\mathrm{t}_{\mathrm{r}} 10.4 \mathrm{~min}(S) ; \mathrm{t}_{\mathrm{r}} 12.9 \mathrm{~min}(R)$ [Chiralpak AD (0.46 cm x $25 \mathrm{~cm}$ ) (from Daicel Chemical Ind., Ltd.)] Hexane/i-PrOH, 95/5, 1.0 $\mathrm{mL} / \mathrm{min}, 100 \%$ ee.

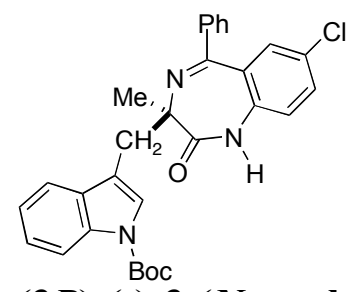

(3R)-(-)-3-(N-tert-butoxycarbonyl-indol-3-yl)methyl-7-chloro-1,3-dihydro-3-methyl-5phenyl-2H-1,4-benzodiazepin-2-one $[(R)-10 c]$

The general procedure was followed with (-)-10d $(78.5 \mathrm{mg}, 0.106 \mathrm{mmol})$ and $1.25 \%$ TFA in $\mathrm{CH}_{2} \mathrm{Cl}_{2}(3.4 \mathrm{~mL})$. The reaction mixture was stirred at r.t. for $2 \mathrm{~h}$. Purification with flash column chromatography on $\mathrm{C} 18\left(90: 10 \mathrm{CH}_{3} \mathrm{OH} / \mathrm{H}_{2} \mathrm{O}\right)$ to afford $54 \mathrm{mg}(99 \%)$ of (-)-10c as colorless semi-solid. $[\alpha]_{\mathrm{D}}{ }^{23}=-108.2\left(\mathrm{c}=1.8, \mathrm{CHCl}_{3}\right){ }^{1} \mathrm{H} \mathrm{NMR}\left(\mathrm{CDCl}_{3}\right): \delta 9.23(\mathrm{~s}, \mathrm{NH}), 8.14(\mathrm{br}, 1 \mathrm{H})$, $7.62(\mathrm{t}, J=8.8 \mathrm{~Hz}, 2 \mathrm{H}), 7.50-7.33(\mathrm{~m}, 6 \mathrm{H}), 7.33-7.26(\mathrm{~m}, 1 \mathrm{H}), 7.24-7.15(\mathrm{~m}, 2 \mathrm{H}), 7.00(\mathrm{~d}, J=$ $8.7 \mathrm{~Hz}, 1 \mathrm{H}), 3.35(\mathrm{AB}$ pattern, $J=14.4 \mathrm{~Hz}, 2 \mathrm{H}), 1.64(\mathrm{~s}, 9 \mathrm{H}), 1.29(\mathrm{~s}, 3 \mathrm{H}) .{ }^{13} \mathrm{C} \mathrm{NMR}\left(\mathrm{CDCl}_{3}\right): \delta$ 174.7, 166.1, 149.8, 140.6, 136.9, 135.2, 132.2, 131.9, 130.5, 130.2, 129.9, 129.2, 128.2, 128.0, 125.3, 124.1, 122.4, 121.4, 120.0, 116.4, 115.1, 83.2, 66.6, 33.6 (broad peak), 20.4 (broad peak). HRMS calcd. for $\mathrm{C}_{30} \mathrm{H}_{29} \mathrm{ClN}_{3} \mathrm{O}_{3}(\mathrm{M}+1)$ 514.1897, found $514.1910(+2.4 \mathrm{ppm},+1.2 \mathrm{mmu})$. HPLC $\mathrm{t}_{\mathrm{r}} 10.8 \mathrm{~min}((-)$-enantiomer, putatively $(R))$; $\mathrm{t}_{\mathrm{r}} 12.6 \mathrm{~min}((+)$-enantiomer, putatively $(S))$ [Chiralpak AD $(0.46 \mathrm{~cm} \times 25 \mathrm{~cm})$ (from Daicel Chemical Ind., Ltd.)] Hexane/i-PrOH , 95/5, 1.0 $\mathrm{mL} / \mathrm{min}, 100 \%$ ee.

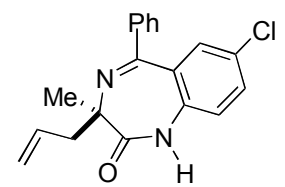

\section{(3R)-(+)-3-allyl-7-chloro-1,3-dihydro-3-methyl-5-phenyl-2H-1,4-benzodiazepin-2-one [ $(R)$ - 11c]}

The general procedure was followed with $(+)-11 d(70.0 \mathrm{mg}, 0.127 \mathrm{mmol})$ and $6.25 \%$ TFA in $\mathrm{CH}_{2} \mathrm{Cl}_{2}(3.4 \mathrm{~mL})$. The reaction mixture was stirred at r.t. for $1.5 \mathrm{~h}$. Purification with preparative TLC on silica gel (8:1:2 $\left.\mathrm{CH}_{2} \mathrm{Cl}_{2} / \mathrm{Et}_{2} \mathrm{O} / \mathrm{Hexane}\right)$ to afford $40.0 \mathrm{mg}(97 \%)$ of $(+)-11 \mathrm{c}$ as colorless semi-solid. $[\alpha]_{\mathrm{D}}{ }^{23}=+45.3\left(\mathrm{c}=1.2, \mathrm{CHCl}_{3}\right){ }^{1} \mathrm{H} \mathrm{NMR}\left(\mathrm{CDCl}_{3}\right): \delta 8.92(\mathrm{~s}, \mathrm{NH}), 7.54-7.34(\mathrm{~m}, 6 \mathrm{H})$, 7.29-7.21 (m, 1H), 7.07-6.99 (m, 1H), 6.09-5.90 (m, 1H), 5.18-5.07 (m, 1H), 5.00 (d, J=16.9Hz, 1H), 2.70-2.55 (m, 1H), 2.51-2.42 (m, 1H), $1.42(\mathrm{~s}, 3 \mathrm{H}) .{ }^{13} \mathrm{C} \mathrm{NMR}\left(\mathrm{CDCl}_{3}\right): \delta 174.3,166.1$, $140.5,136.8,133.7,131.9,130.6,130.3,129.7,129.2,128.4,128.1,121.3,118.6,65.9,41.5$ (broad peak), 22.3 (broad peak). HRMS calcd. for $\mathrm{C}_{19} \mathrm{H}_{18} \mathrm{ClN}_{2} \mathrm{O}(\mathrm{M}+1)$ 325.1108, found 
$325.1106(-0.5 \mathrm{ppm},-0.2 \mathrm{mmu})$. HPLC $\mathrm{t}_{\mathrm{r}} 7.6 \mathrm{~min}((-)$-enantiomer, putatively $(S)) ; \mathrm{t}_{\mathrm{r}} 9.4 \mathrm{~min}((+)-$ enantiomer, putatively $(R))$ [Chiralpak $\mathrm{AD}(0.46 \mathrm{~cm}$ x $25 \mathrm{~cm})$ (from Daicel Chemical Ind., Ltd.)] Hexane/i-PrOH, 90/10, $1.0 \mathrm{~mL} / \mathrm{min}, 98 \%$ ee.<smiles>CCOCC1(C)N=C(c2ccccc2)c2cc(Cl)ccc2NC1=O</smiles>

(3R)-(-)-7-chloro-3-(ethoxycarbonyl)methyl-1,3-dihydro-3-methyl-5-phenyl-2H-1,4benzodiazepin-2-one $[(R)-12 \mathrm{c}]$

The general procedure was followed with $(R)-(-)-\mathbf{1 2 d}(68.0 \mathrm{mg}, 0.114 \mathrm{mmol})$ and $1.25 \%$ TFA in $\mathrm{CH}_{2} \mathrm{Cl}_{2}(3.4 \mathrm{~mL})$. The reaction mixture was stirred at r.t. for $1.5 \mathrm{~h}$. Purification with preparative TLC on silica gel (8:1:2 $\mathrm{CH}_{2} \mathrm{Cl}_{2} / \mathrm{Et}_{2} \mathrm{O} /$ Hexane) to afford $41.3 \mathrm{mg}(98 \%)$ of $(R)-(-)-\mathbf{1 2 c}$ as colorless semi-solid. $[\alpha]_{\mathrm{D}}{ }^{23}=-87.2\left(\mathrm{c}=1.6, \mathrm{CHCl}_{3}\right){ }^{1} \mathrm{H} \mathrm{NMR}\left(\mathrm{CDCl}_{3}\right): \delta 9.64(\mathrm{~s}, \mathrm{NH}), 7.60-7.20$ $(\mathrm{m}, 8 \mathrm{H}), 7.09(\mathrm{~d}, J=8.7 \mathrm{~Hz}, 1 \mathrm{H}), 4.30-4.10(\mathrm{~m}, 2 \mathrm{H}), 2.98$ (AB pattern, $J=15.4 \mathrm{~Hz}, 2 \mathrm{H}), 1.50(\mathrm{~s}$, $3 \mathrm{H}), 1.27(\mathrm{t}, J=7.1 \mathrm{~Hz}, 3 \mathrm{H}) .{ }^{13} \mathrm{C} \mathrm{NMR}\left(\mathrm{CDCl}_{3}\right): \delta 173.6,171.1,166.1,140.3,136.9,132.0$, 130.7, 130.4, 129.8, 128.9, 128.3, 128.2, 121.7, 64.9, 60.4, 43.8, 20.5, 14.3. HRMS calcd. for $\mathrm{C}_{20} \mathrm{H}_{20} \mathrm{ClN}_{2} \mathrm{O}_{3}(\mathrm{M}+1) 371.1162$, found $371.1163(+0.2 \mathrm{ppm},+0.1 \mathrm{mmu})$. HPLC $\mathrm{t}_{\mathrm{r}} 9.7 \mathrm{~min}(S) ; \mathrm{t}_{\mathrm{r}}$ $10.9(R) \min$ [Chiralpak AD $(0.46 \mathrm{~cm} \times 25 \mathrm{~cm})$ (from Daicel Chemical Ind., Ltd.)] Hexane/i$\mathrm{PrOH}, 90 / 10,1.0 \mathrm{~mL} / \mathrm{min}, 99 \%$ ee.

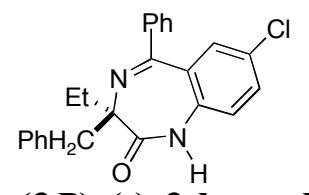

(3R)-(-)-3-benzyl-7-chloro-3-ethyl-1,3-dihydro-3-methyl-5-phenyl-2H-1,4-benzodiazepin-2one $[(R)-20 c]$

Compound (R)-(-)-20d (144.7 mg, $0.236 \mathrm{mmol})$ was dissolved in a solution of 25\% TFA in $\mathrm{CH}_{2} \mathrm{Cl}_{2}(8.0 \mathrm{~mL})$ and stirred for $35 \mathrm{~min}$ at which the starting benzophenone $(R)-(-)-\mathbf{2 0 d}$ was consumed (TLC, 1:3 EtOAc/hexanes). The reaction was quenched with $\mathrm{H}_{2} \mathrm{O}(10 \mathrm{~mL})$ and extracted with $\mathrm{CH}_{2} \mathrm{Cl}_{2}(3 \times 10 \mathrm{~mL})$. The combined organic extracts were dried over anhydrous $\mathrm{Na}_{2} \mathrm{SO}_{4}$, filtered, and concentrated under reduced pressure. The crude product was purified by flash chromatography on silica gel $\left(1^{\text {st }}\right.$ eluent 1:4 EtOAc/hexanes, $2^{\text {nd }}$ eluent 1:10 $\left.\mathrm{Et}_{2} \mathrm{O} / \mathrm{DCM}\right)$ to afford $85.9 \mathrm{mg}(94 \%)$ of $(R)-(-)-20 \mathrm{c}$ as white foam. $[\alpha]_{\mathrm{D}}{ }^{25}=-19.9\left(c 4.62, \mathrm{CHCl}_{3}\right) .{ }^{1} \mathrm{H}$ NMR $\left(\mathrm{CDCl}_{3}\right) \delta 1.04\left(\mathrm{t},{ }^{3} J_{\mathrm{HH}}=6.4 \mathrm{~Hz}, 3 \mathrm{H}\right), 1.69(\mathrm{~m}, 2 \mathrm{H}), 3.11\left(\mathrm{~d},{ }^{2} J_{\mathrm{HH}}=14.0 \mathrm{~Hz}, 1 \mathrm{H}\right), 3.16\left(\mathrm{~d},{ }^{2} J_{\mathrm{HH}}=\right.$ $13.6 \mathrm{~Hz}, 1 \mathrm{H}), 6.99\left(\mathrm{~d},{ }^{3} J_{\mathrm{HH}}=9.2 \mathrm{~Hz}, 1 \mathrm{H}\right), 7.22-7.33(\mathrm{~m}, 6 \mathrm{H}), 7.34-7.43(\mathrm{~m}, 3 \mathrm{H}), 7.45-7.49(\mathrm{~m}$, $1 \mathrm{H}), 7.51-7.53(\mathrm{~m}, 2 \mathrm{H}), 9.19(\mathrm{br}, 1 \mathrm{H}) .{ }^{13} \mathrm{C}$ NMR $\left(\mathrm{CDCl}_{3}\right)$ One conformer $(20$ resonances found for a possible 20 unique carbons): $\delta 8.76,25.70,38.20,70.33,121.58,126.59,127.98,128.04$, $128.31,129.19,129.88,130.23,130.34,131.00,131.88,136.96,137.35,140.92,165.61,174.61$. HRMS (FAB) calcd for $\mathrm{C}_{24} \mathrm{H}_{22} \mathrm{~N}_{2} \mathrm{OCl}[\mathrm{M}+\mathrm{H}]^{+}$: 389.1421 . Found: 389.1410 (-2.8 ppm, -1.1 $\mathrm{mmu})$. HPLC $\mathrm{t}_{\mathrm{r}} 17.9 \mathrm{~min}(R) ; \mathrm{t}_{\mathrm{r}} 20.2 \mathrm{~min}(S)$ [Chiralpak AD-H $(0.46 \mathrm{~cm} \times 25 \mathrm{~cm})$ (from Daicel Chemical Ind., Ltd.) Hexane/ $i$-PrOH, 97/3, $1.0 \mathrm{~mL} / \mathrm{min}, 98 \%$ ee. 


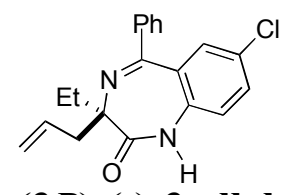

(3R)-(-)-3-allyl-7-chloro-1,3-dihydro-3-ethyl-5-phenyl-2H-1,4-benzodiazepin-2-one [(R)21c]

The general procedure was followed with (-)-21d (21.6 $\mathrm{mg}, 0.0382 \mathrm{mmol})$ and $6.25 \%$ TFA in $\mathrm{CH}_{2} \mathrm{Cl}_{2}(1.2 \mathrm{~mL})$. The reaction mixture was stirred at r.t. for $1.5 \mathrm{~h}$. The crude product was purified by flash chromatography on silica gel $\left(1^{\text {st }}\right.$ eluent 1:4 EtOAc/hexanes, $2^{\text {nd }}$ eluent 1:10 $\mathrm{Et}_{2} \mathrm{O} / \mathrm{DCM}$ ) to afford $12.7 \mathrm{mg}(98 \%)$ of (R)-(-)-21c as colorless semi-solid. [ $\left.\alpha\right]_{\mathrm{D}}{ }^{25}=-25.6$ $\left(c=0.60, \mathrm{CHCl}_{3}\right) .{ }^{1} \mathrm{H}$ NMR $\left(\mathrm{CDCl}_{3}\right): \delta 8.25(\mathrm{~s}, \mathrm{NH}), 7.52-7.51(\mathrm{~m}, 1 \mathrm{H}), 7.45-7.44(\mathrm{~m}, 2 \mathrm{H}), 7.42-$ $7.38(\mathrm{~m}, 2 \mathrm{H}), 7.25-7.24(\mathrm{~m}, 2 \mathrm{H}), 6.96(\mathrm{~d}, J=9 \mathrm{~Hz}, 1 \mathrm{H}), 5.99(\mathrm{~m}, 1 \mathrm{H}), 5.11(\mathrm{~d}, J=9.35 \mathrm{~Hz}, 1 \mathrm{H})$, $5.05(\mathrm{~d}, J=17.3 \mathrm{~Hz}, 1 \mathrm{H}), 2.60(\mathrm{~m}, 2 \mathrm{H}), 1.75(\mathrm{q}, J=7.35 \mathrm{~Hz}, 2 \mathrm{H}), 0.94(\mathrm{t}, J=7.28 \mathrm{~Hz}, 3 \mathrm{H}) .{ }^{13} \mathrm{C}$ $\operatorname{NMR}\left(\mathrm{CDCl}_{3}\right): \delta 173.6,165.6,140.7,136.6,134.0,131.8,130.6,130.3,129.7,129.2,128.4$, 128.1, 121.1, 118.3, 69.4, 37.9, 25.5, 8.3. HRMS calcd. For $\mathrm{C}_{20} \mathrm{H}_{20} \mathrm{ClN}_{2} \mathrm{O}(\mathrm{M}+1) 339.1264$, found $339.1274(+2.9 \mathrm{ppm},+1.0 \mathrm{mmu})$.

\section{F. Hydrolysis of "quaternary" N1-H 1,4-benzodiazepin-2-ones to the corresponding amino acids}

General procedure

The N1-H 1,4-benzodiazepin-2-one $(0.1 \mathrm{mmol})$ to be hydrolyzed was combined with aq. $\mathrm{HCl}$ $(6.0 \mathrm{M}, 1.5 \mathrm{~mL})$ in a pressure tube (Teflon screw cap) and heated at $125^{\circ} \mathrm{C}$ (bath temperature) for 2 days. Water $(2.0 \mathrm{~mL})$ was added and then the mixture was extracted with EtOAc $(5.0 \mathrm{~mL} \mathrm{x}$ $3)$. The water layer was separated, concentrated in vacuo, and the resulting residue was dissolved in EtOH $(2.0 \mathrm{~mL})$. Propylene oxide $(1.0 \mathrm{~mL})$ was added, and the resulting solution was heated at reflux for $30 \mathrm{~min}$. Upon cooling the precipitated white solid was collected and washed with ethyl acetate and acetone, affording the corresponding free amino acid.

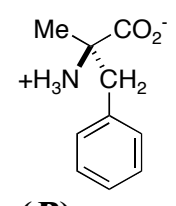

$(R)$ - $\alpha$-methylphenylalanine [26]

$50.1 \mathrm{mg}(0.134 \mathrm{mmol})$ of (+)-2c was treated as above to afford $17.2 \mathrm{mg}(72 \%)$ of $(R)-(-)-\alpha-\mathrm{Me}-$ Phe-OH 26 as white solid. $[\alpha]_{\mathrm{D}}{ }^{25}=+22.5\left(\mathrm{c}=0.12, \mathrm{H}_{2} \mathrm{O}\right)$, lit. $^{11}[\alpha]_{\mathrm{D}}{ }^{25}=-22.1\left(\mathrm{c}=1.0, \mathrm{H}_{2} \mathrm{O}\right)$ for $(S)$ - $\alpha$-methylphenylalanine, We thus assign $(R)$-stereochemistry to our synthesized amino acid.<smiles>CN(C)c1ccc(C(=O)NC(C)(C)C)cc1</smiles>

(R)- $\alpha$-methyl-(4-methylphenyl)alanine [27]

$30.0 \mathrm{mg}(0.077 \mathrm{mmol})$ of (+)-9c was treated as above to afford $11.2 \mathrm{mg}(76 \%)$ of $(R)-(-)-\alpha-\mathrm{Me}-4-$ Me-Phe-OH 27 as a pale solid. $[\alpha]_{\mathrm{D}}{ }^{25}=+16.5\left(\mathrm{c}=0.13, \mathrm{H}_{2} \mathrm{O}\right), \mathrm{lit}^{12}[\alpha]_{\mathrm{D}}{ }^{25}=+16.6\left(\mathrm{c}=0.10, \mathrm{H}_{2} \mathrm{O}\right)$ 
for $(R)$ - $\alpha$-methyl-(4-methylphenyl)alanine, We thus assign $(R)$-stereochemistry to our synthesized amino acid.

${ }_{+\mathrm{H}_{3} \mathrm{Ne}}^{\mathrm{Me}} \mathrm{C}^{\mathrm{C}} \mathrm{CO}_{2} \mathrm{CO}_{2} \mathrm{H}$

(R)- $\alpha$-methylaspartic acid [28]

$31.0 \mathrm{mg}(0.084 \mathrm{mmol})$ of (-)-12c was as above to afford $10.5 \mathrm{mg}(85 \%)$ of $(R)-(-)$ - $\alpha$-Me-aspartic acid 28 as white solid. $[\alpha]_{\mathrm{D}}{ }^{25}=-52.8\left(\mathrm{c}=0.13, \mathrm{H}_{2} \mathrm{O}\right)$, lit. ${ }^{11}[\alpha]_{\mathrm{D}}{ }^{25}=+52.5\left(\mathrm{c}=1.0, \mathrm{H}_{2} \mathrm{O}\right)$ for $(S)-\alpha-$ methylaspartic acid, We thus assign $(R)$-stereochemistry to our synthesized amino acid.

G. Synthesis of $N$-DAM 1,4-benzodiazepin-2,5-diones derived from proline, thioproline, and 4-hydroxyproline<smiles>CON1C(=O)c2ccccc2C(=O)N2CCCC21</smiles>

(11aS)-(+)-10-di(p-anisyl)methyl-2,3,5,10,11,11a-hexahydro-5,11-dioxo-1 $H$-pyrrolo[2,1$c][1,4]$ benzodiazepine $[(S)-(+)-3 d]$

The general method described above was followed with $(S)-(+)-3 c(580 \mathrm{mg}, 2.768 \mathrm{mmol}), \mathrm{NaH}$ (74 mg, $3.10 \mathrm{mmol}, 1.12$ equiv, 60\% suspension in mineral oil) and DAM-Br (2.54 g, 8.33 mmol, 3.0 equiv) in THF $(10.0 \mathrm{~mL})$. Purification with flash chromatography on silica gel (70:30 EtOAc:hexane) provided (3S)-(+)-3d in 93\% yield (1.08 g). Chiral stationary-phase HPLC (Chiralcel AD) indicated $>99.5 \%$ ee. $[\alpha]_{\mathrm{D}}{ }^{26}=+189(\mathrm{c}=1 \mathrm{in} \mathrm{MeOH}) ;{ }^{1} \mathrm{H} \mathrm{NMR}\left(\mathrm{CDCl}_{3}\right) \delta 2.02$ $(\mathrm{m}, 4 \mathrm{H}), 2.70(\mathrm{~m}, 1 \mathrm{H}), 3.58(\mathrm{~m}, 1 \mathrm{H}), 3.74(\mathrm{~s}, 3 \mathrm{H}), 3.80(\mathrm{~s}, 3 \mathrm{H}), 4.20(\mathrm{~m}, 1 \mathrm{H}), 6.60(\mathrm{~s}, 1 \mathrm{H}), 6.73$ $(\mathrm{dd}, J=14.8 \mathrm{~Hz}, J=4.8 \mathrm{~Hz} 7 \mathrm{H}), 6.86(\mathrm{dd}, J=14.8 \mathrm{~Hz}, J=4.8 \mathrm{~Hz}, 4 \mathrm{H}), 7.13$ (m, unassigned aromatic protons $3 \mathrm{H}), 7.78(\mathrm{dd}, J=9.6 \mathrm{~Hz}, J=4.0 \mathrm{~Hz}, 1 \mathrm{H}) ;{ }^{13} \mathrm{C} \mathrm{NMR}\left(\mathrm{CDCl}_{3}\right) \delta$ 23.84, 26.62, 46.45, 55.19, 55.29, 58.11, 113.63, 113.68, 124.53, 126.24, 128.99, 129.74, 130.53, 131.08, 131.34, 131.65, 139.29, 158.65, 158.79, 165.29, 169.42 ; HRMS (FAB) 442.189258 calcd for $\mathrm{C}_{27} \mathrm{H}_{27} \mathrm{~N}_{2} \mathrm{O}_{4}[\mathrm{M}+\mathrm{H}]+$, found 443.19690 (-0.4 ppm, $\left.-0.2 \mathrm{mmu}\right)$.

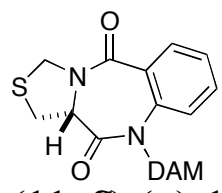

(11aS)-(+)-10-di(p-anisyl)methyl-5,10,11,11a-tetrahydro-5,11-dioxo-1 H,3H$[1,3]$ thiazolo[4,3-c] $[1,4]$ benzodiazepine $[(S)-(+)-30 \mathrm{~d}]$

The DAM group was installed at the $\mathrm{N} 1$ position according to the general method previously described above. $(S)-(+)-30 c(647 \mathrm{mg}, 2.76 \mathrm{mmol})$, NaH (73 mg, $3.10 \mathrm{mmol}, 1.12$ equiv, 60\% suspension in mineral oil) and DAM-Br $(2.53 \mathrm{~g}, 8.33 \mathrm{mmol}, 3.0$ equiv) in THF $(5.0 \mathrm{~mL})$.

Purification with flash chromatography on silica gel (70:30 EtOAc:hexane) provided $288 \mathrm{mg}$ of $(S)-(+)-30 d\left(89 \%\right.$ yield). $[\alpha]_{\mathrm{D}}^{26}=+184.4(\mathrm{c}=1.0$ in MeOH); HLPC (20:80 i-Pr-OH:hexane, CHIRALPAK OD, $4.6 \times 250 \mathrm{~mm}: 1.0 \mathrm{ml} / \mathrm{min}$; UV detection at $254 \mathrm{~nm}): 15.2 \mathrm{~min}, 99.5 \%$ ee; ${ }^{1} \mathrm{H}$ NMR $\left(\mathrm{CDCl}_{3}\right) \delta 3.14(\mathrm{~m}, 1 \mathrm{H}), 3.66(\mathrm{~s}, 3 \mathrm{H}), 3.76(\mathrm{~s}, 3 \mathrm{H}), 3.84(\mathrm{~m}, 1 \mathrm{H}), 4.4(\mathrm{~m}, 1 \mathrm{H}), 4.53(\mathrm{~d}, J$ $=10.8 \mathrm{~Hz}, 1 \mathrm{H}), 4.88(\mathrm{~d}, J=10.8 \mathrm{~Hz}, 1 \mathrm{H}), 6.64(\mathrm{~s}, 1 \mathrm{H}) 6.67(\mathrm{dd}, J=8.8 \mathrm{~Hz}, J=1.6 \mathrm{~Hz} 1 \mathrm{H})$, $6.82(\mathrm{~d}, J=8.8 \mathrm{~Hz}, 2 \mathrm{H}), 7.02(\mathrm{~d}, J=8.8 \mathrm{~Hz}, 2 \mathrm{H}), 7.13(\mathrm{~m}$, unassigned aromatic proton, $6 \mathrm{H})$, $7.76(\mathrm{dd}, J=8.8 \mathrm{~Hz}, J=1.6 \mathrm{~Hz}, 1 \mathrm{H}) ;{ }^{13} \mathrm{C} \mathrm{NMR}\left(\mathrm{CDCl}_{3}\right) \delta 31.55,49.24,55.12,55.23,59.40$, 
67.16, 113.68, 113.80, 124.61, 126.41, 128.95, 129.05, 129.98, 130.10, 130.32, 130.52, 130.78, 130.84, 131.46, 138.74, 158.71, 158.85, 164.51, 167.67 HRMS (FAB) calcd for $\mathrm{C}_{26} \mathrm{H}_{25} \mathrm{~N}_{2} \mathrm{O}_{4} \mathrm{~S}$ $[\mathrm{M}+\mathrm{H}]+461.145679$, found $461.1516(-3.5 \mathrm{ppm},-1.6 \mathrm{mmu})$.

General procedure for TBDMS protection of 4-hydroxyproline-derived 1,4-benzodiazepin2,5-diones 31c and 32c.

31c or 32c ( $0.9 \mathrm{mmol}, 1$ equiv) was combined with tert-butyldimethylsilyl chloride ( $2.0 \mathrm{mmol}$, 2.2 equiv) and imidazole (4.5 mmol, 5.0 equiv) in DMF and stirred at room temperature for 12 hours. The reaction mixture was partitioned between $\mathrm{CH}_{2} \mathrm{Cl}_{2}(2 \times 50 \mathrm{~mL})$ and $\mathrm{H}_{2} \mathrm{O}(50 \mathrm{~mL})$. The organic layers were dried $\left(\mathrm{Na}_{2} \mathrm{SO}_{4}\right)$, filtered and the solvent was evaporated under reduced pressure. The crude was purified using flash column chromatography (50:50 EtOAc:hexane) on silica gel to yield the desired products $33 \mathbf{c}(92 \%)$ and $34 \mathbf{c}(89 \%)$ as white solids.

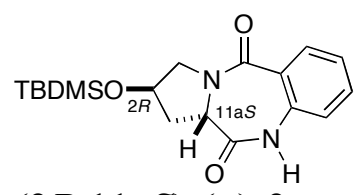

(2R,11aS)-(+)-2-tert-butyldimethylsilyloxy-2,3,5,10,11,11a-hexahydro-5,11-dioxo-1Hpyrrolo[2,1-c][1,4]benzodiazepine [34c]

32c $(213.0 \mathrm{mg}, 0.918 \mathrm{mmol})$ was reacted with TBDMS-Cl (304.0 mg, $2.01 \mathrm{mmol})$ and imidazole (312.9 mg, $4.57 \mathrm{mmol}$ ) in DMF to afford the title compound 34c $(218.0 \mathrm{mg}, 89 \%) .{ }^{1} \mathrm{H} \mathrm{NMR}$ spectroscopy indicated $>98 \%$ de. $[\alpha]_{\mathrm{D}}{ }^{26}=-215.9(\mathrm{c}=1.0$ in $\mathrm{MeOH}) ;{ }^{1} \mathrm{H} \mathrm{NMR}\left(\mathrm{CDCl}_{3}\right) \delta 0.04$ $(\mathrm{s}, 3 \mathrm{H}), 0.05(\mathrm{~s}, 3 \mathrm{H}), 0.82(\mathrm{~s}, 9 \mathrm{H}), 2.05(\mathrm{~m}, 1 \mathrm{H}), 2.80(\mathrm{~m}, 1 \mathrm{H}), 3.72(\mathrm{~m}, 2 \mathrm{H}), 4.19(\mathrm{~m}, 1 \mathrm{H}), 4.57$ $(\mathrm{m}, 1 \mathrm{H}), 6.96(\mathrm{~d}, J=8.0 \mathrm{~Hz}, 1 \mathrm{H}), 7.22(\mathrm{~m}$, unassigned aromatic proton, $1 \mathrm{H}), 7.44(\mathrm{~m}$, unassigned aromatic proton, $1 \mathrm{H}), 7.98(\mathrm{dd}, J=9.2 \mathrm{~Hz}, J=1.6 \mathrm{~Hz}, 1 \mathrm{H}), 8.42(\mathrm{bs}, 1 \mathrm{H}) ;{ }^{13} \mathrm{C} \mathrm{NMR}\left(\mathrm{CDCl}_{3}\right) \delta$ $1.19,-0.09,17.95,25.72,35.45,54.36,55.64,69.39,120.90,125.15,126.79,131.49,132.34$, 135.30, 165.80, 170.92; HRMS (FAB) calcd for $\mathrm{C}_{18} \mathrm{H}_{27} \mathrm{~N}_{2} \mathrm{O}_{3} \mathrm{Si}[\mathrm{M}+\mathrm{H}]+347.171271$, found 347.1768 (-6.6 ppm, $-2.3 \mathrm{mmu})$.

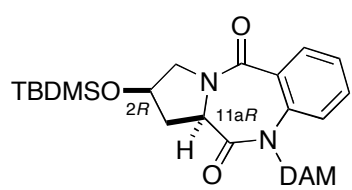

$(2 R, 11 \mathrm{a} R)-(+)-10$-di(p-anisyl)methyl-2-tert-butyldimethylsilyloxy-2,3,5,10,11,11ahexahydro-5,11-dioxo-1 $H$-pyrrolo[2,1-c] [1,4]benzodiazepine [33d]

31c $(212.4 \mathrm{mg}, 0.915 \mathrm{mmol})$ was reacted with TBDMS-Cl $(303.5 \mathrm{mg}, 2.01 \mathrm{mmol})$ and imidazole (311.6 mg, $4.57 \mathrm{mmol})$ in DMF to afford the title compound 33c $(282.0 \mathrm{mg}, 92 \%) .{ }^{1} \mathrm{H} \mathrm{NMR}$ spectroscopy indicated $>98 \%$ de. $[\alpha]_{\mathrm{D}}{ }^{26}=+311.7(\mathrm{c}=1.0$ in $\mathrm{MeOH}) ;{ }^{1} \mathrm{H} \mathrm{NMR}\left(\mathrm{CDCl}_{3}\right) \delta 0.03$ (s, 3H), 0.04 (s, 3H), $0.80(\mathrm{~s}, 9 \mathrm{H}), 2.20(\mathrm{~m}, 1 \mathrm{H}), 2.82(\mathrm{~m}, 1 \mathrm{H}), 3.49$ (dd, $J=17.2 \mathrm{~Hz}, J=5.2 \mathrm{~Hz}$ ,1H), $3.93(\mathrm{dd}, J=17.2 \mathrm{~Hz}, J=5.2 \mathrm{~Hz}, 1 \mathrm{H}), 4.05(\mathrm{~m}, 1 \mathrm{H}), 4.42(\mathrm{~m}, 1 \mathrm{H}), 7.02(\mathrm{~d}, J=8.0 \mathrm{~Hz}$, $1 \mathrm{H}), 7.20(\mathrm{~m}$, unassigned aromatic proton, $1 \mathrm{H}), 7.43(\mathrm{~m}$, unassigned aromatic proton, $1 \mathrm{H}), 7.98$ $(\mathrm{dd}, J=9.2 \mathrm{~Hz}, J=1.6 \mathrm{~Hz}, 1 \mathrm{H}), 9.08(\mathrm{bs}, 1 \mathrm{H}) ;{ }^{13} \mathrm{C} \mathrm{NMR}\left(\mathrm{CDCl}_{3}\right) \delta 0.00,0.80,22.90,30.57$, 39.62, 59.93, 60.89, 74.04, 125.88, 129.68, 130.71, 136.23, 137.52, 140.67, 171.02, 176.56; HRMS (FAB) calcd for $\mathrm{C}_{18} \mathrm{H}_{26} \mathrm{~N}_{2} \mathrm{O}_{3} \mathrm{Si}[\mathrm{M}+\mathrm{H}]+347.171271$, found 347.1768 (-6.6 ppm, -2.3 $\mathrm{mmu})$.

The DAM group was installed at the N1 position according to the general method described above with 33c (935 mg, $2.76 \mathrm{mmol}$ ), $\mathrm{NaH}$ (75 mg, $3.10 \mathrm{mmol}, 1.12$ equiv, 60\% suspension in 
mineral oil) and DAM-Br (2.51 g, $8.33 \mathrm{mmol}, 3.0$ equiv) in THF (10.0 mL). Purification with flash chromatography on silica gel (70:30 EtOAc:hexane) provided $126 \mathrm{mg}$ of the title compound 33d (82\% yield). Proton NMR indicated $>98 \%$ de. $[\alpha]_{\mathrm{D}}{ }^{26}=+214.6(\mathrm{c}=1.0 \mathrm{in}$ $\mathrm{MeOH}) ;{ }^{1} \mathrm{H}$ NMR $\left(\mathrm{CDCl}_{3}\right) \delta 0.00(\mathrm{~s}, 3 \mathrm{H}), 0.08(\mathrm{~s}, 3 \mathrm{H}), 0.86(\mathrm{~s}, 9 \mathrm{H}), 2.57(\mathrm{~m}, 1 \mathrm{H}), 2.84(\mathrm{~m}, 1 \mathrm{H})$, $3.24(\mathrm{~m}, 1 \mathrm{H}), 3.73(\mathrm{~s}, 3 \mathrm{H}), 3.79(\mathrm{~s}, 3 \mathrm{H}), 4.14(\mathrm{~m}, 2 \mathrm{H}), 4.37(\mathrm{~m}, 1 \mathrm{H}), 6.63(\mathrm{~s}, 1 \mathrm{H}), 6.72(\mathrm{dd}, J$ $=8.0 \mathrm{~Hz}, J=1.6 \mathrm{~Hz}, 1 \mathrm{H}), 6.87(\mathrm{~m}$, unassigned aromatic proton, $1 \mathrm{H}), 7.18-7.24(\mathrm{~m}$, unassigned aromatic proton, $9 \mathrm{H}), 7.76(\mathrm{dd}, J=8.0 \mathrm{~Hz}, J=1.6 \mathrm{~Hz}, 1 \mathrm{H}) ;{ }^{13} \mathrm{C} \mathrm{NMR}\left(\mathrm{CDCl}_{3}\right) \delta 0.00,0.76$, 22.93, 30.64, 39.36, 57.40, 60.06, 61.48, 72.23, 74.31, 118.57, 118.74, 129.33, 131.18, 132.66, 134.05, 134.76, 135.33, 135.36, 135.46, 136.09, 144.17, 163.55, 163.71, 170.65, 173.47 HRMS (FAB) calcd for $\mathrm{C}_{33} \mathrm{H}_{41} \mathrm{~N}_{2} \mathrm{O}_{5} \mathrm{Si}[\mathrm{M}+\mathrm{H}]+574.270651$, found 574.1259 (-1.2 ppm, $-0.5 \mathrm{mmu}$ ).

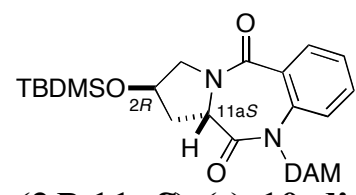

$(2 R, 11 \mathrm{a} S)-(-)-10$-di(p-anisyl)methyl-2-tert-butyldimethylsilyloxy-2,3,5,10,11,11a-hexahydro5,11-dioxo-1 $H$-pyrrolo[2,1-c][1,4]benzodiazepine [34d]

The DAM group was installed at the N1 position according to the general method previously described above with 34c (934 mg, $2.76 \mathrm{mmol}$ ), $\mathrm{NaH}$ (75 mg, $3.10 \mathrm{mmol}, 1.12$ equiv, 60\% suspension in mineral oil) and DAM-Br (2.54 g, $8.33 \mathrm{mmol}, 3.0$ equiv) in THF (10.0 mL). Purification with flash chromatography on silica gel (70:30 EtOAc:hexane) provided $137 \mathrm{mg}$ of the title compound 34d (89\% yield). Proton NMR indicated $>98 \%$ de $[\alpha]_{\mathrm{D}}{ }^{26}=-190.1(\mathrm{c}=1.0$ in $\mathrm{MeOH}) ;{ }^{1} \mathrm{H}$ NMR $\left(\mathrm{CDCl}_{3}\right) \delta 0.03(\mathrm{~s}, 6 \mathrm{H}), 0.81(\mathrm{~s}, 9 \mathrm{H}), 1.98(\mathrm{~m}, 1 \mathrm{H}), 2.80(\mathrm{~m}, 1 \mathrm{H}), 3.56(\mathrm{~m}, 1 \mathrm{H})$, $3.68(\mathrm{~s}, 3 \mathrm{H}), 3.76(\mathrm{~s}, 3 \mathrm{H}), 4.30(\mathrm{~m}, 1 \mathrm{H}), 4.57(\mathrm{~m}, 1 \mathrm{H}), 6.63(\mathrm{~s}, 1 \mathrm{H}), 6.67(\mathrm{dd}, J=8.0 \mathrm{~Hz}, J=1.6$ $\mathrm{Hz}, 1 \mathrm{H}), 6.82(\mathrm{~m}$, unassigned aromatic proton, $1 \mathrm{H}), 6.90-7.15(\mathrm{~m}$, unassigned aromatic proton, 9H), $7.74(\mathrm{dd}, J=8.0 \mathrm{~Hz}, J=1.6 \mathrm{~Hz}, 1 \mathrm{H}) ;{ }^{13} \mathrm{C} \mathrm{NMR}\left(\mathrm{CDCl}_{3}\right) \delta-0.02,0.96,20.13,32.61,40.36$, 57.30, 60.03, 62.48, 71.01, 74.31, 118.57, 118.74, 131.33, 133.18, 133.66, 134.05, 134.06, 135.33, 135.36, 135.46, 136.09, 144.17, 163.55, 165.71, 170.85, 173.47 ; HRMS (FAB) calcd for $\mathrm{C}_{33} \mathrm{H}_{41} \mathrm{~N}_{2} \mathrm{O}_{5} \mathrm{Si}[\mathrm{M}+\mathrm{H}]+574.270651$, found 574.1259 (-1.2 ppm, $\left.-0.5 \mathrm{mmu}\right)$.

\section{H. Enantioselective deprotonation/alkylation of $N$-DAM 1,4-benzodiazepin-2,5-diones derived from proline, thioproline, and 4-hydroxyproline}

General in situ protocol for C3-alkylation At $-100{ }^{\circ} \mathrm{C}$ under nitrogen, to a stirred solution of the 1,4-benzodiazepin-2,5-dione ( $0.15 \mathrm{mmol}, 1.0$ equiv) and additive (if desired, HMPA, 0.90 mmol, 6.0 equiv) in anhydrous THF $(3.0 \mathrm{~mL})$ the electrophile was added (1.5 mmol, 10 equiv) and the mixture was stirred for $15 \mathrm{~min}$. KHMDS (0.15 mmol, 1.2 equiv, $0.5 \mathrm{M})$ was then added via syringe and the mixture stirred at $-100{ }^{\circ} \mathrm{C}$ until the starting benzodiazepine was consumed (TLC). The reaction was quenched at $-100{ }^{\circ} \mathrm{C}$ by the addition of saturated aqueous $\mathrm{NH}_{4} \mathrm{Cl}(5.0$ $\mathrm{mL})$ and extracted with $\mathrm{CH}_{2} \mathrm{Cl}_{2}(3 \times 10 \mathrm{~mL})$. The combined extracts were dried over anhydrous $\mathrm{Na}_{2} \mathrm{SO}_{4}$, filtered, and concentrated. The crude product was purified by flash column chromatography on silica gel (70:30 EtOAc:hexanes). 


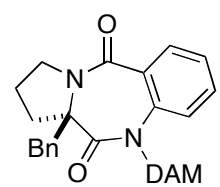

(11aR)-(+)-10-di(p-anisyl)methyl-11a-benzyl-2,3,5,10,11,11a-hexahydro-5,11-dioxo-1Hpyrrolo[2,1-c] $[1,4]$ benzodiazepine $[(R)-(+)-35 d]$

The in situ method described above was followed with compound $(S)-(+)-3 \mathbf{d}(66.3 \mathrm{mg}, 0.15$ mmol, 1.0 equiv), HMPA ( $160 \mu \mathrm{L}, 0.90 \mathrm{mmol}, 6.0$ equiv), KHMDS (300 $\mu \mathrm{L}, 0.15 \mathrm{mmol}, 1.2$ equiv, $0.5 \mathrm{M}$ in hexanes) and $\mathrm{BnBr}(142 \mu \mathrm{L}, 1.5 \mathrm{mmol}, 10$ equiv) in anhydrous THF (5.0 mL). Purification with flash column chromatography (70:30 EtOAc:hexane) gave $78 \mathrm{mg}$ of $(R)-(+)-$ 35d $(98 \%$ yield $) .[\alpha]_{\mathrm{D}}{ }^{26}=+200.3(\mathrm{c}=1.0 \mathrm{in} \mathrm{MeOH}) ;{ }^{1} \mathrm{H} \mathrm{NMR}\left(\mathrm{CDCl}_{3}\right) \delta 1.59(\mathrm{~m}, 2 \mathrm{H}), 2.00(\mathrm{~m}$, $1 \mathrm{H}), 2.73(\mathrm{~d}, J=13.5 \mathrm{~Hz}, 1 \mathrm{H}), 2.77(\mathrm{~d}, J=13.5 \mathrm{~Hz}, 1 \mathrm{H}) 3.01(\mathrm{~m}, 1 \mathrm{H}), 3.39(\mathrm{~m}, 1 \mathrm{H}), 3.81(\mathrm{~s}$, $3 \mathrm{H}), 3.82(\mathrm{~s}, 3 \mathrm{H}), 4.04(\mathrm{~m}, 1 \mathrm{H}), 6.41(\mathrm{~s}, 1 \mathrm{H}), 6.89(\mathrm{~d}, J=7.5 \mathrm{~Hz}, 2 \mathrm{H}) 7.02-7.34(\mathrm{~m}$, unassigned aromatic protons $14 \mathrm{H}), 7.98(\mathrm{dd}, J=9.7 \mathrm{~Hz}, J=1.6 \mathrm{~Hz}, 1 \mathrm{H}) ;{ }^{13} \mathrm{C} \mathrm{NMR}\left(\mathrm{CDCl}_{3}\right) \delta 21.66,37.37$, $40.07,49.63,55.19,69.67,69.79,113.30,113.66,123.78,126.38,127.13,128.45,128.97$, $129.79,130.00,130.29,130.81,130.86,131.744,131.91,131.12,139.98,158.47,158.71,165.48$, 170.22; HRMS (FAB) calcd for $\mathrm{C}_{34} \mathrm{H}_{31} \mathrm{~N}_{2} \mathrm{O}_{4}[\mathrm{M}+\mathrm{H}]+533.236208$, found $533.24261(-2.7$ ppm, -1.4 mmu). Chiral stationary-phase HPLC (Chiralcel AD) indicated 98\% ee.

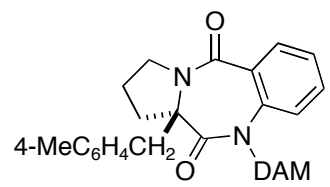

(11aR)-(+)-10-di(p-anisyl)methyl-2,3,5,10,11,11a-hexahydro-11a-(4-methyl)benzyl-5,11dioxo-1H-pyrrolo[2,1-c][1,4]benzodiazepine $[(R)-(+)-36 \mathrm{~d}]$

The in situ method described above was followed with compound $(S)-(+)-3 \mathbf{d}(66.7 \mathrm{mg}, 0.15$ mmol, 1.0 equiv), HMPA (160 $\mu \mathrm{L}, 0.90$ mmol, 6.0 equiv), KHMDS (300 $\mu \mathrm{L}, 0.15$ mmol, 1.2 equiv, $0.5 \mathrm{M}$ in hexanes) and 4-methyl-benzyl bromide (275 $\mu \mathrm{L}, 1.5 \mathrm{mmol}, 10$ equiv) in anhydrous THF (5.0 mL). Purification with flash column chromatography (70:30 EtOAc:hexane) gave $76 \mathrm{mg}$ of $(R)-(+)-36 d$ (93\% yield). $[\alpha]_{\mathrm{D}}{ }^{26}=130.1(\mathrm{c}=1.0 \mathrm{in} \mathrm{MeOH}) ;{ }^{1} \mathrm{H} \mathrm{NMR}\left(\mathrm{CDCl}_{3}\right) \delta$ $1.42(\mathrm{~m}, 2 \mathrm{H}), 1.99(\mathrm{~m}, 1 \mathrm{H}), 2.32(\mathrm{~s}, 3 \mathrm{H}), 2.63(\mathrm{~d}, J=14.0 \mathrm{~Hz}, 1 \mathrm{H}), 2.72(\mathrm{~d}, J=14.0 \mathrm{~Hz}, 1 \mathrm{H})$, $2.99(\mathrm{~m}, 2 \mathrm{H}), 3.36(\mathrm{~m}, 1 \mathrm{H}), 3.78(\mathrm{~s}, 3 \mathrm{H}), 3.79(\mathrm{~s}, 3 \mathrm{H}), 6.38(\mathrm{~s}, 1 \mathrm{H}), 6.77(\mathrm{~m}$, unassigned aromatic protons $5 \mathrm{H}), 7.53(\mathrm{~m}$, unassigned aromatic protons $10 \mathrm{H}), 7.95(\mathrm{dd}, J=9.6 \mathrm{~Hz}, J=2.0 \mathrm{~Hz}, 1 \mathrm{H})$; ${ }^{13} \mathrm{C} \mathrm{NMR}\left(\mathrm{CDCl}_{3}\right) \delta$ 21.04, 21.69, 37.43, 39.69, 55.16, 69.74, 73.35, 113.26, 113.60, 123.80, 126.34, 128.99, 129.13, 129.66, 129.98, 130.30, 130.78, 131.66, 131.89, 131.97, 136.71, 139.91, 158.45, 158.66, 170.32; HRMS (FAB) calcd for $\mathrm{C}_{35} \mathrm{H}_{35} \mathrm{~N}_{2} \mathrm{O}_{4}[\mathrm{M}+\mathrm{H}]+547.251858$, found $547.26190(+4.1 \mathrm{ppm},+2.2 \mathrm{mmu})$. Chiral stationary-phase HPLC (Chiralcel AD) indicated $>99.5 \%$ ee.

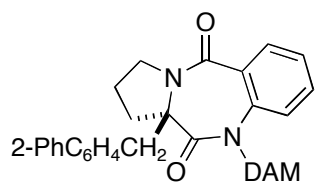

(11aR)-(+)-10-di(p-anisyl)methyl-2,3,5,10,11,11a-hexahydro-5,11-dioxo-11a-(2phenyl)benzyl-1 $H$-pyrrolo[2,1-c][1,4]benzodiazepine $[(R)-(+)-37 \mathrm{~d}]$

The in situ method described above was followed with compound $(S)-(+)-3 \mathbf{d}(66.1 \mathrm{mg}, 0.15$ mmol, 1.0 equiv), HMPA (160 $\mu \mathrm{L}, 0.90$ mmol, 6.0 equiv), KHMDS (300 $\mu \mathrm{L}, 0.15$ mmol, 1.2 
equiv, $0.5 \mathrm{M}$ in hexanes) and 2-phenylbenzyl bromide ( $370 \mu \mathrm{L}, 1.5 \mathrm{mmol}, 10$ equiv) in anhydrous THF $(5.0 \mathrm{~mL})$. Purification with flash column chromatography (70:30 EtOAc:hexane) gave $88 \mathrm{mg}$ of $(R)-(+)-37 \mathbf{d}(94 \%$ yield $) .[\alpha]_{\mathrm{D}}{ }^{26}=+86.7(\mathrm{c}=1.0 \mathrm{in} \mathrm{MeOH}) ;{ }^{1} \mathrm{H} \mathrm{NMR}\left(\mathrm{CDCl}_{3}\right) \delta$ $1.29(\mathrm{~m}, 2 \mathrm{H}), 1.47(\mathrm{~m}, 1 \mathrm{H}), 2.52(\mathrm{~m}, 1 \mathrm{H}), 2.70(\mathrm{~d}, J=14.8 \mathrm{~Hz}, 1 \mathrm{H}), 2.77(\mathrm{~d}, J=14.8 \mathrm{~Hz}, 1 \mathrm{H})$, $3.25(\mathrm{~m}, 1 \mathrm{H}), 3.74(\mathrm{~s}, 3 \mathrm{H}), 3.75(\mathrm{~s}, 3 \mathrm{H}), 3.85(\mathrm{~m}, 1 \mathrm{H}), 6.37(\mathrm{~s}, 1 \mathrm{H}), 6.73(\mathrm{~m}$, unassigned aromatic protons $4 \mathrm{H}$ ), 7.03 (m, unassigned aromatic protons $15 \mathrm{H}), 7.82(\mathrm{dd}, J=9.6 \mathrm{~Hz}, J=21.6 \mathrm{~Hz}, 1 \mathrm{H})$; ${ }^{13} \mathrm{C} \mathrm{NMR}\left(\mathrm{CDCl}_{3}\right) \delta$ 21.48, 35.30, 36.12, 49.47, 55.18, 69.62, 70.06, 113.34, 113.70, 123.69, $126.33,126.78,127.07,127.50,128.25,128.94,129.19,129.37,129.92,130.38,130.52,131.01$, $131.77,131.84,133.05,139.82,141.08,142.95,158.49,158.74,170.28$; HRMS (FAB) calcd for $\mathrm{C}_{40} \mathrm{H}_{37} \mathrm{~N}_{2} \mathrm{O}_{4}[\mathrm{M}+\mathrm{H}]+609.267508$, found 609.1228 (-0.4 ppm, $-0.1 \mathrm{mmu}$ ). Chiral stationaryphase HPLC (Chiralcel AD) indicated 99\% ee.

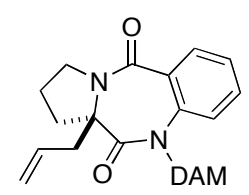

(11aR)-(+)-11a-allyl-10-di(p-anisyl)methyl-2,3,5,10,11,11a-hexahydro-5,11-dioxo-1Hpyrrolo[2,1-c][1,4]benzodiazepine $[(R)-(+)-38 \mathrm{~d}]$

The in situ method described above was followed with compound $(S)-(+)-3 d(66.0 \mathrm{mg}, 0.15$ mmol, 1.0 equiv), HMPA ( $160 \mu \mathrm{L}, 0.90 \mathrm{mmol}, 6.0$ equiv), KHMDS ( $300 \mu \mathrm{L}, 0.15 \mathrm{mmol}, 1.2$ equiv, $0.5 \mathrm{M}$ in hexanes) and allyl-Br $(176 \mu \mathrm{L}, 1.5 \mathrm{mmol}, 10$ equiv) in anhydrous THF $(5.0 \mathrm{~mL})$. Purification with flash column chromatography (70:30 EtOAc:hexane) gave $45 \mathrm{mg}$ of $(R)-(+)-$ 38d $(65 \%$ yield $)$. $[\alpha]_{\mathrm{D}}{ }^{26}=+340.0(\mathrm{c}=1.0 \mathrm{in} \mathrm{MeOH}) ;{ }^{1} \mathrm{H}$ NMR $\left(\mathrm{CDCl}_{3}\right) \delta 1.30(\mathrm{~m}, 1 \mathrm{H}), 1.78(\mathrm{~m}$, $1 \mathrm{H}), 1.92(\mathrm{~m}, 1 \mathrm{H}), 2.09(\mathrm{~m}, 1 \mathrm{H}), 2.21(\mathrm{~m}, 1 \mathrm{H}), 3.04(\mathrm{~m}, 1 \mathrm{H}), 3.44(\mathrm{~m}, 1 \mathrm{H}), 3.76(\mathrm{~s}, 3 \mathrm{H}), 3.79(\mathrm{~s}$, $3 \mathrm{H}), 4.01(\mathrm{~m}, 1 \mathrm{H}), 4.75(\mathrm{~d}, J=16.8 \mathrm{~Hz}, 1 \mathrm{H}), 5.09(\mathrm{~d}, J=10.4 \mathrm{~Hz}, 1 \mathrm{H}), 5.54(\mathrm{M}, 1 \mathrm{H}), 6.49(\mathrm{~s}$, $1 \mathrm{H}), 6.76(\mathrm{~m}$, unassigned aromatic protons $4 \mathrm{H}), 7.21$ (m, unassigned aromatic protons, $7 \mathrm{H}), 7.82$ $(\mathrm{dd}, J=9.5 \mathrm{~Hz}, J=1.6 \mathrm{~Hz} 1 \mathrm{H}) ;{ }^{13} \mathrm{C} \mathrm{NMR}\left(\mathrm{CDCl}_{3}\right) \delta 21.72,36.95,38.70,49.53,54.02,68.35$, $113.35,113.60,119.67,123.47,126.06,129.05,130.07,130.25,130.50,131.17,131.51,131.65$, 138.92, 158.66 165.36, 170.43; HRMS (FAB) calcd for $\mathrm{C}_{30} \mathrm{H}_{31} \mathrm{~N}_{2} \mathrm{O}_{4}[\mathrm{M}+\mathrm{H}]+, 483.220558$ found 483.22745 (-2.1 ppm, $-1.0 \mathrm{mmu}$ ). Chiral stationary-phase HPLC (Chiralcel AD) indicated $93 \%$ ee.

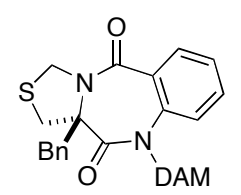

(11aS)-(+)-10-di(p-anisyl)methyl-11a-benzyl-5,10,11,11a-tetrahydro-5,11-dioxo-1 $H, 3 H-$ $[1,3]$ thiazolo[4,3-c] $[1,4]$ benzodiazepine $[(S)-(+)-39 \mathrm{~d}]$

The general in situ protocol described above implemented without HMPA: $(S)-(+)-30 d(69.0 \mathrm{mg}$, $0.15 \mathrm{mmol}, 1.0$ equiv), KHMDS ( $300 \mu \mathrm{L}, 0.15 \mathrm{mmol}, 1.2$ equiv, $0.5 \mathrm{M}$ in hexanes) and $\mathrm{BnBr}$ $(143 \mu \mathrm{L}, 1.5 \mathrm{mmol}, 10$ equiv) in anhydrous THF $(3.0 \mathrm{~mL})$. Purification with flash column chromatography (70:30 EtOAc:hexane) gave $79 \mathrm{mg}$ of $(S)-(+)-39 d$ (98\% yield). $[\alpha]_{\mathrm{D}}{ }^{26}=+89.7$ (c $=1.0$ in $\mathrm{MeOH}) ;{ }^{1} \mathrm{H} \mathrm{NMR}\left(\mathrm{CDCl}_{3}\right) \delta 3.62(\mathrm{~d}, J=14.4 \mathrm{~Hz}, 1 \mathrm{H}), 3.69(\mathrm{~d}, J=14.4 \mathrm{~Hz}, 1 \mathrm{H})$, $3.70(\mathrm{~s}, 3 \mathrm{H}), 3.74(\mathrm{~s}, 3 \mathrm{H}), 4.49$ (d, $J=10.8 \mathrm{~Hz}, 1 \mathrm{H}), 4.97$ (d, $J=10.8 \mathrm{~Hz}, 1 \mathrm{H}), 5.42(\mathrm{~d}, J=1.2 \mathrm{~Hz}$, $1 \mathrm{H}), 5.70(\mathrm{~d}, J=1.2 \mathrm{~Hz}, 1 \mathrm{H}), 6.44(\mathrm{~s}, 1 \mathrm{H}) 6.77(\mathrm{~m}, 4 \mathrm{H}), 7.20-7.32(\mathrm{~m}, 10 \mathrm{H}), 7.07(\mathrm{~d}, J=8.8 \mathrm{~Hz}$, $2 \mathrm{H}), 7.13(\mathrm{~m}$, unassigned aromatic proton, $6 \mathrm{H}), 7.76(\mathrm{dd}, J=8.8 \mathrm{~Hz}, J=1.6 \mathrm{~Hz}, 1 \mathrm{H}) ;{ }^{13} \mathrm{C}$ NMR $\left(\mathrm{CDCl}_{3}\right) \delta 35.78,51.12,55.17,67.59,94.77,113.53,113.79,117.85,123.47,126.18,126.96$, 
128.36, 129.14, 129.71, 130.07, 130.24, 131.50; HRMS (FAB) calcd for $\mathrm{C}_{33} \mathrm{H}_{31} \mathrm{~N}_{2} \mathrm{O}_{4} \mathrm{~S}[\mathrm{M}+\mathrm{H}]+$ 550.184805 , found $550.1255(+4.1 \mathrm{ppm},+2.2 \mathrm{mmu})$. Chiral stationary-phase HPLC (Chiralcel OD) indicated $99 \%$ ee.

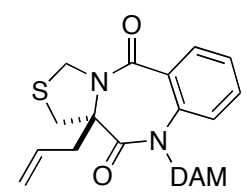

(11aS)-(+)-11a-allyl-10-di(p-anisyl)methyl-5,10,11,11a-tetrahydro-5,11-dioxo-1H,3H$[1,3]$ thiazolo[4,3-c] $[1,4]$ benzodiazepine $[(S)-(+)-40 \mathrm{~d}]$

The general in situ protocol described above was implemented without HMPA: $(S)-(+)-30 d(70.1$ $\mathrm{mg}, 0.15 \mathrm{mmol}, 1.0$ equiv), KHMDS (300 $\mu \mathrm{L}, 0.15 \mathrm{mmol}, 1.2$ equiv, $0.5 \mathrm{M}$ in hexanes) and allyl-Br (176 $\mu \mathrm{L}, 1.5 \mathrm{mmol}, 10$ equiv) in anhydrous THF $(3.5 \mathrm{~mL})$. Purification with flash column chromatography (70:30 EtOAc:hexane) gave $66 \mathrm{mg}$ of $(S)-(+)-40 d(89 \%$ yield $) ..[\alpha]_{\mathrm{D}}{ }^{26}=$ $+102.7(\mathrm{c}=1.0$ in $\mathrm{MeOH}) ;{ }^{1} \mathrm{H}$ NMR $\left(\mathrm{CDCl}_{3}\right) \delta 3.10(\mathrm{~m}, 2 \mathrm{H}), 3.74(\mathrm{~s}, 3 \mathrm{H}), 3.75(\mathrm{~m}, 3 \mathrm{H}), 4.57$ (d, $J=14.0 \mathrm{~Hz}, 1 \mathrm{H}) 4.84(\mathrm{~d}, J=14.0 \mathrm{~Hz}, 1 \mathrm{H}), 5.08(\mathrm{~d}, J=14.0 \mathrm{~Hz}, 1 \mathrm{H}), 5.20(\mathrm{~d}, J=14.0 \mathrm{~Hz}, 1 \mathrm{H})$, $5.47(\mathrm{~d}, J=1.6 \mathrm{~Hz}, 1 \mathrm{H}), 5.69$ (d, $J=1.6 \mathrm{~Hz}, 1 \mathrm{H}), 5.69(\mathrm{~m}, 1 \mathrm{H}), 6.41(\mathrm{~s}, 1 \mathrm{H}), 6.79-6.82(\mathrm{~m}$, unassigned aromatic proton, $3 \mathrm{H}), 7.14-7.28(\mathrm{~m}$, unassigned aromatic proton, $8 \mathrm{H}), 7.66(\mathrm{dd}, J=$ $7.6 \mathrm{~Hz}, J=2 \mathrm{~Hz}, 1 \mathrm{H}) ;\left(\mathrm{CDCl}_{3}\right) \delta 31.35,38.41,50.05,53.12,56.23,60.20,68.23,113.68$, $113.80,114.52,125.61,126.71,129.05,130.15,129.98,130.10,130.32,130.52,130.78,130.84$, 131.46, 138.36, 138.74, 158.71, 159.85, 165.51, 170.01. HRMS (FAB) calcd for $\mathrm{C}_{29} \mathrm{H}_{29} \mathrm{~N}_{2} \mathrm{O}_{4} \mathrm{~S}$ $[\mathrm{M}+\mathrm{H}]+500.176980$, found $500.1747(+4.5 \mathrm{ppm},-2.3 \mathrm{mmu})$. Chiral stationary-phase HPLC (Chiralcel OD) indicated 94\% ee.

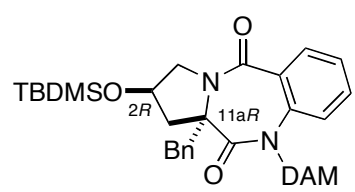

$(2 R, 11 \mathrm{a} R)-(-)-10$-di(p-anisyl)methyl-11a-benzyl-2-tert-butyldimethylsilyloxy-2,3,5,10,11,11ahexahydro-5,11-dioxo-1 $H$-pyrrolo[2,1-c][1,4]benzodiazepine [41d]

The general in situ protocol described above was implemented without HMPA: 33d $(86.0 \mathrm{mg}$, $0.15 \mathrm{mmol}, 1.0$ equiv), KHMDS (300 $\mu \mathrm{L}, 0.15 \mathrm{mmol}, 1.2$ equiv, $0.5 \mathrm{M}$ in hexanes) and $\mathrm{BnBr}$ (144 $\mu \mathrm{L}, 1.5 \mathrm{mmol}, 10$ equiv) in anhydrous THF (4.0 mL). Purification with flash column chromatography (70:30 EtOAc:hexane) gave $94 \mathrm{mg}$ of (-)-41d (95\% yield). $[\alpha]_{\mathrm{D}}{ }^{26}=-148.4$ (c = 1.0 in $\mathrm{MeOH}$ ); HLPC (20:80 i-Pr-OH:hexane, CHIRALPAK AD, 4.6 x $250 \mathrm{~mm}: 1.0 \mathrm{ml} / \mathrm{min}$; UV detection at $254 \mathrm{~nm}): 10.1 \mathrm{~min}$, diastereomer ratio of 41d:42d was 96:4; ${ }^{1} \mathrm{H} \mathrm{NMR}\left(\mathrm{CDCl}_{3}\right)$ $\delta-0.12(\mathrm{~s}, 3 \mathrm{H}),-0.04(\mathrm{~s}, 3 \mathrm{H}), 0.77(\mathrm{~s}, 9 \mathrm{H}), 2.20(\mathrm{~m}, 1 \mathrm{H}), 2.48(\mathrm{~d}, J=14.4 \mathrm{~Hz}, 1 \mathrm{H}), 2.91(\mathrm{~d}, J$ $=14.4 \mathrm{~Hz}, 1 \mathrm{H}) 3.48(\mathrm{~m}, 2 \mathrm{H}), 3.69(\mathrm{~s}, 3 \mathrm{H}), 3.74(\mathrm{~m}, 1 \mathrm{H}), 3.78(\mathrm{~s}, 3 \mathrm{H}), 6.67(\mathrm{~s}, 1 \mathrm{H}), 6.69(\mathrm{dd}, J$ $=8.0 \mathrm{~Hz}, J=1.6 \mathrm{~Hz}, 1 \mathrm{H}), 6.84-7.22(\mathrm{~m}$, unassigned aromatic proton, $16 \mathrm{H}), 7.89(\mathrm{dd}, J=7.6 \mathrm{~Hz}, J$ $=2 \mathrm{~Hz}, 1 \mathrm{H}) ;{ }^{13} \mathrm{C} \mathrm{NMR}\left(\mathrm{CDCl}_{3}\right) \delta 0.00,0.22,23.17,30.92,44.31,49.14,60.28,60.33,60.41$, 60.46, 61.51, 71.72, 73.02, 74.27, 74.44, 118.78, 118.82, 118.88, 118.94, 129.28, 131.29, 132.53, 133.70, 133.87, 134.27,134.43, 134.98, 135.12, 135.22, 135.30, 135.54, 135.84, 135.89, 136.17, 136.50, 140.19, 143.75, 163.70, 163.87, 170.83, 175.91 HRMS (FAB) calcd for $\mathrm{C}_{40} \mathrm{H}_{47} \mathrm{~N}_{2} \mathrm{O}_{5} \mathrm{Si}$ $[\mathrm{M}+\mathrm{H}]+662.31760$, found $663.3255(+0.1 \mathrm{ppm},+0.1 \mathrm{mmu})$. 


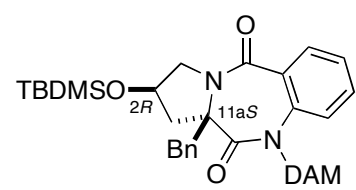

$(2 R, 11 \mathrm{aS})-(+)-10$-di(p-anisyl)methyl-11a-benzyl-2-tert-butyldimethylsilyloxy-

2,3,5,10,11,11a-hexahydro-5,11-dioxo-1 $H$-pyrrolo[2,1-c][1,4]benzodiazepine [42d]

The general in situ protocol described above was implemented without HMPA: 34d (85.2 mg, $0.15 \mathrm{mmol}, 1.0$ equiv), KHMDS (300 $\mu \mathrm{L}, 0.15 \mathrm{mmol}, 1.2$ equiv, $0.5 \mathrm{M}$ in hexanes) and $\mathrm{BnBr}$ (143 $\mu \mathrm{L}, 1.5 \mathrm{mmol}, 10$ equiv) in anhydrous THF (4.0 mL). Purification with flash column chromatography (70:30 EtOAc:hexane) gave $48 \mathrm{mg}$ of $(+)-\mathbf{4 2 d}\left(49 \%\right.$ yield). $[\alpha]_{\mathrm{D}}{ }^{26}=+250.1(\mathrm{c}=$ 1.0 in $\mathrm{MeOH})$; HLPC (20:80 i-Pr-OH:hexane, CHIRALPAK AD, 4.6 x $250 \mathrm{~mm}: 1.0 \mathrm{ml} / \mathrm{min}$; UV detection at $254 \mathrm{~nm}): 11.6 \mathrm{~min}$, diastereomer ratio of 42d:41d was 92:8; ${ }^{1} \mathrm{H} \mathrm{NMR}\left(\mathrm{CDCl}_{3}\right)$ $\delta-0.04(\mathrm{~s}, 3 \mathrm{H}),-0.03(\mathrm{~s}, 3 \mathrm{H}), 0.79(\mathrm{~s}, 9 \mathrm{H}), 2.02(\mathrm{~m}, 1 \mathrm{H}), 2.67(\mathrm{~d}, J=14.4 \mathrm{~Hz}, 1 \mathrm{H}), 2.85(\mathrm{~d}, J$ $=14.4 \mathrm{~Hz}, 1 \mathrm{H}) 3.05(\mathrm{~m}, 1 \mathrm{H}), 3.26(\mathrm{~m}, 1 \mathrm{H}), 3.73(\mathrm{~s}, 3 \mathrm{H}), 3.78(\mathrm{~s}, 3 \mathrm{H}), 4.01(\mathrm{~m}, 1 \mathrm{H}), 6.18(\mathrm{~s}, 1 \mathrm{H})$, $6.72(\mathrm{dd}, J=8.0 \mathrm{~Hz}, J=1.6 \mathrm{~Hz}, 1 \mathrm{H}), 6.81-7.34$ (m, unassigned aromatic proton, $16 \mathrm{H}), 7.90$ (dd, $J$ $=7.6 \mathrm{~Hz}, J=2 \mathrm{~Hz}, 1 \mathrm{H}) ;{ }^{13} \mathrm{C} \mathrm{NMR}\left(\mathrm{CDCl}_{3}\right) \delta 0.00,0.06,23.10,30.83,45.96,50.17,60.18$, $60.25,60.30,72.34,74.33,75.77,118.17,118.77,128.80,131.76,132.31,133.58,134.16$, $134.31,134.87,135.10,135.19,135.62,137.75,137.12,137.15,140.06,145.46,163.62,163.82$, 170.74, 174.88 HRMS (FAB) calcd for $\mathrm{C}_{40} \mathrm{H}_{47} \mathrm{~N}_{2} \mathrm{O}_{5} \mathrm{Si}[\mathrm{M}+\mathrm{H}]+662.31760$, found 663.3255 (+0.1ppm, $+0.1 \mathrm{mmu})$.

\section{Removal of N-DAM group from quaternary 1,4-benzodiazepin-2,5-diones General procedure}

The benzodiazepin-2,5-dione $(0.1 \mathrm{mmol})$ was combined with trifluoroacetic acid $(2.5 \mathrm{~mL})$ and dichloromethane $(7.5 \mathrm{~mL})$. The reaction was stirred at ambient temperature for 3 hours. Volatiles were removed under reduced pressure to afford an orange oil which was purified by preparatory thin layer chromatography (80:20 EtOAc:hexanes).<smiles>O=C1Nc2ccccc2C(=O)N2CC[C@H]1C2</smiles>

(11aR)-(+)-11a-benzyl-2,3,5,10,11,11a-hexahydro-5,11-dioxo-1 $H$-pyrrolo[2,1$c][1,4]$ benzodiazepine $[(R)-(+)-35 c]$

Compound $(3 R)-(+)-35 d(60.0 \mathrm{mg}, 0.10 \mathrm{mmol})$ was treated as described above to quantitatively yield the desired compound $(+)-35 \mathbf{c}$. $(R)$-Stereochemistry was subsequently established by hydrolysis to $(R)-(-)$-benzylproline: $[\alpha]_{\mathrm{D}}{ }^{26}=+105.6(\mathrm{c}=1.0$ in $\mathrm{MeOH}) ;{ }^{1} \mathrm{H} \mathrm{NMR}\left(\mathrm{CDCl}_{3}\right) \delta 1.64$ $(\mathrm{m}, 2 \mathrm{H}), 2.08(\mathrm{~m}, 1 \mathrm{H}), 2.84(\mathrm{~d}, J=14.0 \mathrm{~Hz}, 1 \mathrm{H}), 2.85$ (d, $J=14.0 \mathrm{~Hz}, 1 \mathrm{H}), 2.95$ (m, 1H), 3.49 $(\mathrm{m}, 1 \mathrm{H}), 4.01(\mathrm{~m}, 1 \mathrm{H}), 6.98(\mathrm{~m}$, unassigned aromatic protons $3 \mathrm{H}), 7.27(\mathrm{~m}$, unassigned aromatic protons $5 \mathrm{H}), 7.52(\mathrm{t}, J=8.0 \mathrm{~Hz}, 1 \mathrm{H}), 8.32(\mathrm{bs}, 1 \mathrm{H}) ;{ }^{13} \mathrm{C} \mathrm{NMR}\left(\mathrm{CDCl}_{3}\right) \delta 21.46,35.33,39.29$, $50.49,68.19,119.74,125.09,126.47,127.37,128.63,129.85,131.57,132.94,134.81,135.12$, 165.19, 172.02; HRMS (FAB) calcd for $\mathrm{C}_{19} \mathrm{H}_{19} \mathrm{~N}_{2} \mathrm{O}_{2}[\mathrm{M}+\mathrm{H}]+307.136828$, found 307.1329 ($1.0 \mathrm{ppm},-0.8 \mathrm{mmu})$. 


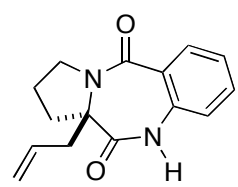

(11a $R)-(+)-11$ a-allyl-2,3,5,10,11,11a-hexahydro-5,11-dioxo-1 $H$-pyrrolo[2,1c] $[1,4]$ benzodiazepine $[(R)-(+)-38 c]$

Compound $(3 R)-(+)-38 d(60.0 \mathrm{mg}, 0.10 \mathrm{mmol})$ was treated as described above to afford compound (+)-38c in $96 \%$ yield. $[\alpha]_{\mathrm{D}}{ }^{26}=+54.2(\mathrm{c}=1.0$ in $\mathrm{MeOH}) ;{ }^{1} \mathrm{H} \mathrm{NMR}\left(\mathrm{CDCl}_{3}\right) \delta 1.80$ $(\mathrm{m}, 1 \mathrm{H}), 1.93(\mathrm{~m}, 1 \mathrm{H}), 2.02(\mathrm{~m}, 1 \mathrm{H}), 2.10(\mathrm{~m}, 1 \mathrm{H}), 2.32(\mathrm{~m}, 1 \mathrm{H}), 3.09(\mathrm{~m}, 1 \mathrm{H}), 3.58(\mathrm{~m}, 1 \mathrm{H})$, $3.60(\mathrm{~m}, 1 \mathrm{H}), 4.04(\mathrm{~m}, 1 \mathrm{H}), 4.90(\mathrm{~d}, J=16.8 \mathrm{~Hz}, 1 \mathrm{H}), 5.12(\mathrm{dd}, J=10.8 \mathrm{~Hz}, J=0.8 \mathrm{~Hz}, 1 \mathrm{H})$, $5.78(\mathrm{~m}, 1 \mathrm{H}), 6.98(\mathrm{~d}, J=8.0 \mathrm{~Hz}, 1 \mathrm{H}), 7.29(\mathrm{~m}$, unassigned aromatic proton $1 \mathrm{H}), 7.49(\mathrm{~m}$, unassigned aromatic proton, $1 \mathrm{H}), 8.04(\mathrm{dd}, J=8.8 \mathrm{~Hz}, J=1.2 \mathrm{~Hz}, 1 \mathrm{H}), 8.34(\mathrm{bs}, 1 \mathrm{H}) ;{ }^{13} \mathrm{C} \mathrm{NMR}$ $\left(\mathrm{CDCl}_{3}\right) \delta 21.68,35.49,38.15,50.40,66.95,119.58,120.41,124.83,126.11,130.69,131.51$, 132.76, 135.12, 165.13, 172.11); HRMS (FAB) calcd for $\mathrm{C}_{15} \mathrm{H}_{17} \mathrm{~N}_{2} \mathrm{O}_{2}[\mathrm{M}+\mathrm{H}]+257.113353$, found $257.1284(-1.9 \mathrm{ppm},-0.5 \mathrm{mmu})$.

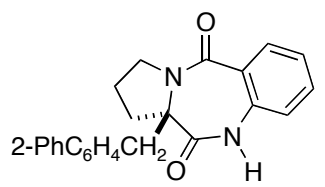

(11aR)-(-)-11a-(2-phenyl)benzyl-2,3,5,10,11,11a-hexahydro-5,11-dioxo-1 $H$-pyrrolo[2,1c] $[1,4]$ benzodiazepine $[(R)-(-)-37 c]$

Compound (3R)-(+)-37d (14.2 mg, $0.023 \mathrm{mmol})$ was treated as described above to afford compound (-)-37c in $99 \%$ yield. $[\alpha]_{\mathrm{D}}{ }^{25}=-12.3\left(\mathrm{c}=0.4\right.$ in $\left.\mathrm{CHCl}_{3}\right) ;{ }^{1} \mathrm{H}$ NMR $\left(\mathrm{CDCl}_{3}\right): \delta 1.26-1.33$ $(\mathrm{m}, 2 \mathrm{H}), 1.52-1.56(\mathrm{~m}, 1 \mathrm{H}), 2.50-2.53(\mathrm{~m}, 1 \mathrm{H}), 2.88-2.91(\mathrm{~d}, J=14.4 \mathrm{~Hz}, 1 \mathrm{H}), 2.97-3.01(\mathrm{~d}, J=$ $14.4 \mathrm{~Hz}, 1 \mathrm{H}), 3.42-3.45(\mathrm{~m}, 1 \mathrm{H}), 3.82-2.83(\mathrm{~m}, 1 \mathrm{H}), 6.90-6.92(\mathrm{dd}, J=1.2 \mathrm{~Hz}, 0.8 \mathrm{~Hz}, 1 \mathrm{H})$, 7.02-7.04 (m, 1H), 7.13-7.15 (m, 2H), 7.17-7.20 (m, 1H), 7.24-7.26 (m, 1H), 7.29-7.31 (m, 1H), 7.34-7.40 (m, 3H), 7.48-7.52 (td, $J=1.6 \mathrm{~Hz}, 2 \mathrm{~Hz}, 1.6 \mathrm{~Hz}, 1 \mathrm{H}), 8.03$ (s, NH), 8.08-8.10 (dd, $J=$ $2 \mathrm{~Hz}, 1.6 \mathrm{~Hz}, 1 \mathrm{H}) .{ }^{13} \mathrm{C} \mathrm{NMR}\left(\mathrm{CDCl}_{3}\right): \delta 171.72,165.12,143.22,141.23,134.92,132.83,132.52$, $131.48,130.45,129.58,129.54,128.46,127.63,127.14,127.11,126.32,124.98,119.70,68.59$, $50.29,34.42$ (2C accidentally equivalent), 21.16. HRMS calcd. For $\mathrm{C}_{25} \mathrm{H}_{23} \mathrm{~N}_{2} \mathrm{O}_{2}(\mathrm{M}+1)$ 383.1760 , found $383.1769(+2.5 \mathrm{ppm},+1.0 \mathrm{mmu})$

\section{J. Table of HPLC retention times}

\begin{tabular}{ccccc}
\hline compound & column & $\begin{array}{c}\text { solvent, flow } \\
\text { rate }\end{array}$ & $\begin{array}{c}\text { fast enantiomer } \\
\text { retention time } \\
\text { (config) }\end{array}$ & $\begin{array}{c}\text { slow } \\
\text { enantiomer } \\
\text { retention time } \\
\text { (config) }\end{array}$ \\
\hline 1d & AD & $\begin{array}{c}\text { Hexane/i- } \\
\text { PrOH, } 90 / 10,\end{array}$ & $10.6 \min (R)$ & $16.4 \mathrm{~min}(S)$ \\
& & $1.0 \mathrm{~mL} / \mathrm{min}$ & & \\
2c & AD & Hexane/i- & $11.3 \min (S)$ & $14.5 \min (R)$ \\
& & PrOH, $95 / 5$, & & \\
& & $1.0 \mathrm{~mL} / \mathrm{min}$ & &
\end{tabular}


Hexane/i-

$\mathrm{PrOH}, 92 / 8$,

$10.9 \min (S)$

$13.1 \min (R)$

$1.0 \mathrm{~mL} / \mathrm{min}$

OD Hexane/ $i$ -

$\mathrm{PrOH}, 99 / 1$,

$22.1(S)$

$24.7(R)$

$1.0 \mathrm{~mL} / \mathrm{min}$

3d

AD

Hexane $/ i-\mathrm{PrOH}$

$80 / 20,1$

$\mathrm{mL} / \mathrm{min}$

$5 d$

OD

Hexane/i-PrOH

97/3, 1 mL/min

6d

OD

Hexane/i-PrOH

99/1, 1mL/min

7d

OD

Hexane/i-PrOH

99/1, $1 \mathrm{~mL} / \mathrm{min}$

8d

OD

9c

AD

$\mathrm{Hexane} / \mathrm{i}-\mathrm{PrOH}$

99/1, $1 \mathrm{~mL} / \mathrm{min}$

Hexane/i-

$\mathrm{PrOH}, 95 / 5$,

$1.0 \mathrm{~mL} / \mathrm{min}$

9d

AD

Hexane/i-

$\mathrm{PrOH}, 98 / 2$,

10c

Ad

$1.0 \mathrm{~mL} / \mathrm{min}$,

Hexane/i-

$\mathrm{PrOH}, 95 / 5$,

$1.0 \mathrm{~mL} / \mathrm{min}$

10d

AD

Hexane/i-

$\mathrm{PrOH}, 97 / 3$,

$1.0 \mathrm{~mL} / \mathrm{min}$

11c

AD

Hexane/i-

PrOH, 90/10,

11d

OD

$1.0 \mathrm{~mL} / \mathrm{min}$

Hexane/i-

PrOH, 99/1,

12c

AD

$1.0 \mathrm{~mL} / \mathrm{min}$,

Hexane/i-

PrOH, 90/10,

$1.0 \mathrm{~mL} / \mathrm{min}$

12d

$\mathrm{AD}$

Hexane/i-

$\mathrm{PrOH}, 90 / 10$,

13d

$1.0 \mathrm{~mL} / \mathrm{min}$

$\mathrm{AD}$

Hexane / i-

$\mathrm{PrOH}$, 98/2, $1.0 \mathrm{~mL} / \mathrm{min}$

$18.6 \min (3 R)$

$21.1 \min (3 S)$

$16.3 \min (R)$

$19.7 \min (S)$

$40.2 \min (S)$

$46.7 \min (R)$

$25.1 \min (R)$

$28.9 \min (S)$

$41.6 \min (S)$

$46.9 \min (R)$

$10.4 \min (S)$

$12.9 \min (R)$

$30.8 \mathrm{~min}(S)$;

$41.3 \min (R)$

$10.8 \min ((-)-$ enantiomer, putatively $(R))$ $13.5 \mathrm{~min}((-)-$ enantiomer, putatively $(R)$ ) $7.6 \min ((-)-$ enantiomer, putatively $(S)$ ) $18.4 \min ((-)-$ enantiomer, putatively $(S)$ );

$9.7 \min (S)$

$11.0 \mathrm{~min}(S)$;

$26.1 \min ((+)-$ enantiomer, putatively $(R)$ );
$13.1 \mathrm{~min}(R)$

$31.4 \min ((-)-$ enantiomer,

$12.6 \min ((+)-$ enantiomer, putatively $(S)$ ) $17.6 \min ((+)-$ enantiomer, putatively $(S)$ ) $9.4 \min ((+)-$ enantiomer, putatively $(R)$ ) $22.1 \min ((+)-$ enantiomer, putatively $(R)$ ) $10.9 \min (R)$ putatively $(S)$ ) 


\begin{tabular}{|c|c|c|c|c|}
\hline 14d & $\mathrm{AD}$ & $\begin{array}{c}\text { Hexane/i- } \\
\text { PrOH , 90/10, } \\
1.0 \mathrm{~mL} / \mathrm{min} .\end{array}$ & $\begin{array}{c}9.9 \text { min }((-)- \\
\text { enantiomer, } \\
\text { putatively }(S))\end{array}$ & $\begin{array}{c}13.2 \min ((+)- \\
\text { enantiomer, } \\
\text { putatively }(R))\end{array}$ \\
\hline $15 d$ & $\mathrm{AD}$ & $\begin{array}{l}\text { Hexane/i- } \\
\mathrm{PrOH}, 92 / 8 \\
1.0 \mathrm{~mL} / \mathrm{min}\end{array}$ & $\begin{array}{l}8.4 \text { min }((-)- \\
\text { enantiomer, } \\
\text { putatively }(S))\end{array}$ & $\begin{array}{c}10.7 \text { min }((+)- \\
\text { enantiomer, } \\
\text { putatively }(R))\end{array}$ \\
\hline $16 d$ & $\mathrm{AD}$ & $\begin{array}{c}\text { Hexane/i- } \\
\mathrm{PrOH}, 90 / 10, \\
1.0 \mathrm{~mL} / \mathrm{min}\end{array}$ & $\begin{array}{l}13.4 \text { min }((+)- \\
\text { enantiomer, } \\
\text { putatively }(S))\end{array}$ & $\begin{array}{l}18.1 \text { min }((-)- \\
\text { enantiomer, } \\
\text { putatively }(R))\end{array}$ \\
\hline $17 d$ & OD & $\begin{array}{l}\text { Hexane/i- } \\
\text { PrOH , 97/3, } \\
\text { 1mL/min }\end{array}$ & $16.3 \min (R)$ & $19.7 \min (S)$ \\
\hline $18 d$ & $\mathrm{AD}$ & $\begin{array}{l}\text { Hexane/i- } \\
\mathrm{PrOH}, 97 / 3, \\
1 \mathrm{~mL} / \mathrm{min}\end{array}$ & $22.1 \min (S)$ & $31.0 \min (R)$ \\
\hline 19d & OD & $\begin{array}{l}\text { Hexane/i- } \\
\mathrm{PrOH}, 99 / 1, \\
\text { 1mL/min }\end{array}$ & $26.5 \min (R)$ & $32.7 \min (S)$ \\
\hline 20c & AD-H & $\begin{array}{l}\text { Hexane/i- } \\
\text { PrOH, 97/3, } 1 \\
\text { mL/min }\end{array}$ & $17.9 \min (R)$ & $20.2 \min (S)$ \\
\hline 20d & $\mathrm{AD}$ & $\begin{array}{l}\text { Hexane/i- } \\
\text { PrOH , 97/3, } \\
\text { 1mL/min }\end{array}$ & $27.2 \min (S)$ & $34.6 \min (R)$ \\
\hline 21d & OD & $\begin{array}{l}\text { Hexane/i- } \\
\mathrm{PrOH}, 99 / 1, \\
\text { 1mL/min }\end{array}$ & $21.2 \min (S)$ & $23.7 \min (R)$ \\
\hline 22d & OD & $\begin{array}{l}\text { Hexane/i- } \\
\mathrm{PrOH}, 99 / 1, \\
1 \mathrm{~mL} / \mathrm{min}\end{array}$ & $21.5 \min (R)$ & $26.5 \min (S)$ \\
\hline 23d & $\mathrm{AD}$ & $\begin{array}{l}\text { Hexane/i- } \\
\mathrm{PrOH}, 97 / 3, \\
1 \mathrm{~mL} / \mathrm{min}\end{array}$ & $20.5 \min (R)$ & $25.1 \min (S)$ \\
\hline 24d & AD-H & $\begin{array}{l}\text { Hexane/i- } \\
\mathrm{PrOH}, 97 / 3, \\
1 \mathrm{~mL} / \mathrm{min}\end{array}$ & $16.7 \min (S)$ & $20.7 \min (R)$ \\
\hline $25 d$ & OD & $\begin{array}{l}\text { Hexane/i- } \\
\mathrm{PrOH}, 90 / 10, \\
1 \mathrm{~mL} / \mathrm{min}\end{array}$ & $9.0 \min (R)$ & $14.3 \min (S)$ \\
\hline 35d & OD & $\begin{array}{c}\text { Hexane } / i-\mathrm{PrOH} \\
80 / 20,1 \\
\mathrm{~mL} / \mathrm{min}\end{array}$ & $8.3 \min (3 S)$ & $9.8 \min (3 R)$ \\
\hline 36d & OD & $\begin{array}{c}\text { Hexane } / i-\mathrm{PrOH} \\
80 / 20,1 \\
\mathrm{~mL} / \mathrm{min}\end{array}$ & $11.0 \min (3 S)$ & $13.5 \min (3 R)$ \\
\hline
\end{tabular}




\begin{tabular}{|c|c|c|c|c|}
\hline 37d & OD & $\begin{array}{c}\text { Hexane } / i-\mathrm{PrOH} \\
80 / 20,1 \\
\mathrm{~mL} / \mathrm{min}\end{array}$ & $7.8 \min (3 R)$ & 10.1 Min $(3 S)$ \\
\hline 38d & OD & $\begin{array}{c}\text { Hexane } / i \text {-PrOH } \\
80 / 20,1 \\
\mathrm{~mL} / \mathrm{min}\end{array}$ & $9.2 \min (3 S)$ & $11.3 \min (3 R)$ \\
\hline 39d & OD & $\begin{array}{c}\text { Hexane } / i-\mathrm{PrOH} \\
80 / 20,1 \\
\mathrm{~mL} / \mathrm{min}\end{array}$ & $\begin{array}{l}13.4 \text { min }((-)- \\
\text { enantiomer, } \\
\text { putatively }(R))\end{array}$ & $\begin{array}{l}15.8 \text { min }((+)- \\
\text { enantiomer, } \\
\text { putatively }(S))\end{array}$ \\
\hline 40d & OD & $\begin{array}{c}\text { Hexane } / i-\mathrm{PrOH} \\
80 / 20,1 \\
\mathrm{~mL} / \mathrm{min}\end{array}$ & $\begin{array}{c}7.9 \text { min }((-)- \\
\text { enantiomer, } \\
\text { putatively }(R))\end{array}$ & $\begin{array}{l}9.3 \text { min }((+)- \\
\text { enantiomer, } \\
\text { putatively }(S))\end{array}$ \\
\hline 41d & $\mathrm{AD}$ & $\begin{array}{c}\text { Hexane } / i-\mathrm{PrOH} \\
80 / 20,1 \\
\mathrm{~mL} / \mathrm{min}\end{array}$ & $\begin{array}{c}10.1 \min (2 R \\
11 \mathrm{a} R)\end{array}$ & Not applicable \\
\hline $42 d$ & $\mathrm{AD}$ & $\begin{array}{c}\text { Hexane } / i-\mathrm{PrOH} \\
80 / 20,1 \\
\mathrm{~mL} / \mathrm{min}\end{array}$ & $\begin{array}{c}11.6 \min (2 R, \\
11 \mathrm{a} S)\end{array}$ & Not applicable \\
\hline
\end{tabular}

Chiralpak AD $(0.46 \mathrm{~cm} \times 25 \mathrm{~cm})$ (from Daicel Chemical Ind., Ltd.)

Chiralcel OD $(0.46 \mathrm{~cm} \times 25 \mathrm{~cm})$ (from Daicel Chemical Ind., Ltd.)]

\section{K. References}

[1] Šunjić, V.; Kajfež, F.; Štromar, I.; Blažević, N.; Kolbah, D. J. Heterocyclic Chem. 1973, 10, 591-599.

[2] Hart, B. R.; Rush, D. J.; Shea, K. J. J. Am. Chem. Soc. 2000, 122, 460-465.

[3] Kolbah, D.; Blažević, N.; Hannoun, M.; Kajfež, F.; Kovac, T.; Rendic, S.; Šunjić, V.

Helv. Chim. Acta 1977, 60, 265-283.

[4] Lattmann, E.; Billington, D. C.; Poyner, D. R.; Arayarat, P.; Howitt, S. B.; Lawrence, S.; Offel, M. Drug Des. Disc. 2002, 18, 9-21.

[5] Wright, W. B.; Brabander, H. J.; Greenblatt, E. N.; Day, I. P.; Hardy Jr., R. A. J. Med.

Chem. 1978, 21, 1087-1089.

[6] Dyatkin, A. B.; Maryanoff, B. E. Org. Prep. Proc. Int. 2002, 34, 652-655.

[7] Ohtake, Y; Naito, A.; Naito, K.; Matsukawa, H.; Saito, Y.; Toyofuko, H. Preparation and formulation of biphenyl moiety-containing heterocyclic compounds as vasopressin antagonists. PCT Int. Patent Appl. WO 9843976, October 10, 1998.

[8] Peña, M. R.; Stille, J. K. J. Am. Chem. Soc. 1989, 111, 5417-5424.

[9] Liu, R.; Zhang, P.; Gan, T.; Cook, J. M. J. Org. Chem. 1997, 62, 7447-7456.

[10] Abramovitch, R. A.; Chellathurai, T.; Holcomb, W. D.; MacMaster, I. T.; Vanderpool, D. P. J. Org. Chem. 1977, 42, 2920-2926.

[11] Abellán, T.; Chinchilla, R.; Galindo, N.; Guillena, G.; Nájera, C.; Sansano, J. M. Eur. J. Org. Chem. 2000, 2689-2697.

[12] Carlier, P. R.; Zhao, H.; DeGuzman, J.; Lam, P. C.-H. J. Am. Chem. Soc. 2003, 125, 11482-11483. 
